Pacific Journal of Mathematics

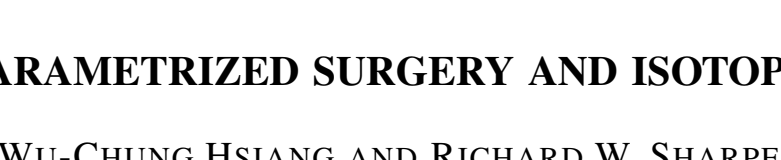




\title{
PARAMETRIZED SURGERY AND ISOTOPY
}

\author{
W. C. Hsiang and R. W. Sharpe
}

The pseudo-isotopy techniques of Cerf-Hatcher-Wagoner are combined with surgery theory to give information about the group of isotopy classes of diffeomorphisms of a smooth manifold. For example, for the $n$-torus, $n \geqq 6$, this group is determined completely. We also provide a geometric interpretation of the periodicity sequence of [11].

Introduction. Let $M$ be an $n$-dim $(n \geqq 6)$ smooth manifold without boundary ${ }^{1}$ and let Diff $M$ be the group of diffeomorphisms of $M$. Let Aut $M$ be the $H$-space of simple homotopy equivalences of $M$ to itself, i.e., Aut $M=\left\{f \in M^{M} \mid f\right.$ is a simple homotopy equivalence $\}$. We have the following fibration

$$
\mathrm{S}(M) \longrightarrow \text { Diff } M \longrightarrow \text { Aut } M \text {. }
$$

Then, a point in $\mathbb{S}(M)$ is represented by a pair $\left(\phi, \phi_{t}\right)$ where $\phi \in \operatorname{Diff} M$ and $\phi_{t}$ is a path in Aut $M$ connecting $\phi$ to Id $\in$ Aut $M$. Set

$$
M_{\phi}=M \times I /\{(m, 1) \sim(\phi(m), 0)\},
$$

the mapping torus of $\dot{\phi} . \phi_{t}$ induces a simple homotopy equivalence

$$
F:\left(M_{\phi}, M \times 1\right) \longrightarrow\left(M \times S^{1}, M \times 1\right) .
$$

Following [10], one can construct a space $\mathscr{S}\left(M \times\left(S^{1}, 1\right)\right)$ of simple homotopy smoothings of $M \times S^{1}$ which are standard on $M \times 1$. W. Browder [1] studies the map

$$
\tau: \mathbb{S}(M) \longrightarrow \mathscr{S}\left(M \times\left(S^{1}, 1\right)\right)
$$

defined by $\tau\left(\left(\phi, \phi_{t}\right)\right)=F$. On the other hand, we have the map

$$
\eta: \mathscr{S}\left(M \times\left(S^{1}, 1\right)\right) \longrightarrow G / 0^{\Sigma M^{+}}
$$

where $\Sigma M^{+}=M \times S^{1} / M \times 1$. Let us consider the following diagram of fibrations

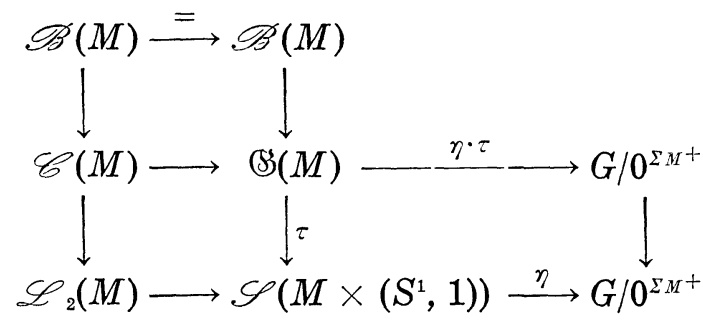

1 Everything works for PL, topological manifold, and manifold with boundary if the boundary is only allowed to move by an isotopy. Using a result of $\mathrm{K}$. Igusa, everything works also for $n=5$. 
where $\mathscr{B}(M), \mathscr{C}(M), \mathscr{L}_{2}(M)$ are the homotopy fibres of the obvious maps. It is easy to see that $\pi_{i}\left(\mathscr{L}_{2}(M)\right)=L_{n+i+2}\left(\pi_{1} M, w\right)$ where $w$ is the first Stiefel-Whitney class of $M$ [13]. It follows from (6) that there is a braid $^{2}$

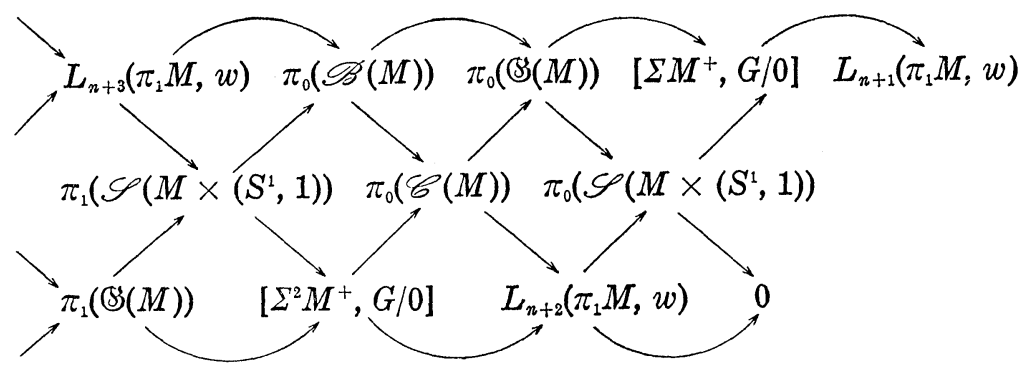

where $\Sigma^{2} M^{+}=M \times D^{2} / M \times S^{1}$ and $\pi_{0}\left(\mathscr{S}\left(M \times\left(S^{1}, 1\right)\right)\right.$ is just the simple homotopy smoothings of $M \times S^{1}$ which are standard on $M \times 1$.

In this paper, we shall identify the elements in $\pi_{i}(\mathscr{C}(M))$ as parametrized surgery problems, and then give an algebraic description of the exact sequence

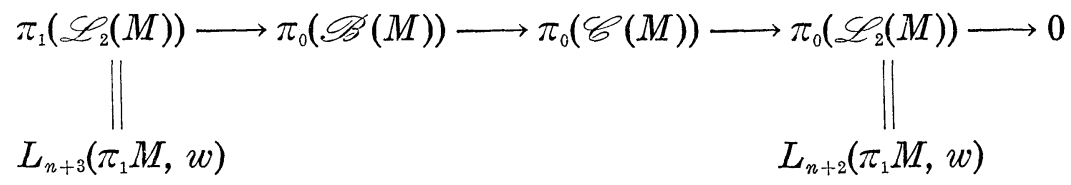

which sits in the braid (7). The entire work is a derivative of Cerf [2] and Hatcher-Wagoner [3], [4]. In particular, the computation of $\pi_{0}(\mathscr{B}(M))$ is an application of the obstruction theory of [3]. On the other hand, (8) can be also viewed as the geometric interpretation of the periodicity sequence of [11] for the case $n$ even. (Cf. Novikov [9] and Giffen [5].)

One can get information about $\pi_{0}(\mathbb{S}(M))$ via another sequence in the braid

$$
\left[\Sigma^{2} M^{+}, G / 0\right] \longrightarrow \pi_{0}(\mathscr{C}(M)) \longrightarrow \pi_{0}(\mathscr{S}(M)) \longrightarrow\left[\Sigma M^{+}, G / 0\right] .
$$

Since $G / 0$ is an infinite loop space, the above sequence is not hard to handle. What one would like to do is to derive information about $\pi_{i}($ Diff $M)(i=0,1)$ via the exact sequence

$$
\begin{aligned}
\pi_{1}(\text { Diff } M) & \longrightarrow \pi_{1}(\text { Aut } M) \stackrel{\partial}{\longrightarrow} \pi_{0}(\$(M)) \\
\longrightarrow & \pi_{0}(\text { Diff } M) \longrightarrow \pi_{0}(\text { Aut } M) .
\end{aligned}
$$

Unfortunately, $\pi_{1}($ Aut $M)$ is not easy to compute and the map $\partial$ may

${ }^{2}$ One can easily extend the braid to left, but we shall concentrate on this part of the braid. 
not behave well as pointed out to us by Hatcher. We shall content ourselves with some computations of simple examples: $M=$ torus $T^{n}$, or a closed manifold which is a product of a compact space with finite fundamental group and a compact $K(\pi, 1)$. The computation of $\pi_{0}$ (Diff $\left.T^{n}\right)$ is actually complete and has also been obtained by Hatcher. We also give some simple examples to show that $\pi_{i}($ Diff $M) \otimes Q i \geqq 0$ can be of infinite rank. All these facts show that the homotopy type of Diff $M$ can be rather bad, even though $M$ is very simple.

In particular, we give negative answers to two problems posed in Manifolds-Tokyo 1973:

(A) Problem 3.4 The answer is almost invariably 'no.' (Cf. Example 1 of $\S$ II.) In other words, most compact $K(\pi, 1)$ 's have many homotopic (even pseudo-isotopic) self-homeomorphisms which are not isotopic.

(B) Problem 3.5 The identity component of the homeomorphism group of $T^{n}(n \geqq 6)$ is not even of the homotopy type of a finite complex. (Cf. Theorem 2.5 and the remarks after the statement of the theorem.)

We are indebted to A. Hatcher for many helpful discussions.

I. Algebraic preliminaries, Let $\pi$ be a group (written multiplicatively) and let $w: \pi \rightarrow\{ \pm 1\}$ be a homomorphism. Let $\Lambda=\mathbf{Z}[\pi]$ be the integral group ring of $\pi$. We define the symbol '-' by

$$
(\Sigma n(g) g)^{-}=\Sigma w(g) n(g) g^{-1}
$$

for elements in $\Lambda$, and then $\Lambda$ becomes a ring with involution. Let $E(\Lambda)$ denote the corresponding group of elementary matrices and define $K_{2}(\Lambda)$ by the exact sequence

$$
i \longrightarrow K_{2}(\Lambda) \longrightarrow \operatorname{St}(\Lambda) \longrightarrow E(\Lambda) \longrightarrow 1
$$

of the universal central extension of $E(\Lambda)$ [8]. Let $W( \pm \pi) \subset S t(\Lambda)$ denote the subgroup generated by all elements of the form $w_{i j}(u)=$ $x_{i j}(u) x_{j i}\left(-u^{-1}\right) x_{i j}(u)$ where $u \in \pm \pi$. Set $W_{0}( \pm \pi)=W( \pm \pi) \cap K_{2}(\Lambda)$. Define

$$
W h_{2}(\pi)=K_{2}(\Lambda) / W_{0}( \pm \pi) \text {. }
$$

The involution '- induces an automorphism

$$
-: W h_{2}(\pi) \longrightarrow W h_{2}(\pi) \text {. }
$$

Let us first recall some definitions and facts from [11] [12]. Let $\widetilde{S U}(\Lambda)$ be the split unitary group with $\varepsilon$ symmetry $(\varepsilon= \pm 1)$ and let $\widetilde{E U}(\Lambda)=[\widetilde{S U}(\Lambda), \widetilde{S U}(\Lambda)]$ be the commutator subgroup of 
$\widetilde{S U}(\Lambda)$. We define $K U_{2}^{\epsilon}(\Lambda)$ by the exact sequence

$$
1 \longrightarrow K U_{2}^{\epsilon}(\Lambda) \longrightarrow \widetilde{S t U}(\Lambda) \longrightarrow \widetilde{E U}(\Lambda) \longrightarrow 1
$$

of the universal central extension of $\widetilde{E U}(\Lambda)$. We have two natural homomorphisms ${ }^{3}$,

$$
\begin{aligned}
& h: S t(\Lambda) \longrightarrow \widetilde{S t U}(\Lambda), \\
& k: \overparen{S t U}(\Lambda) \longrightarrow S t(\Lambda) \text {. }
\end{aligned}
$$

Next, set

$$
\begin{aligned}
& S t(\Lambda)^{\prime}=S t(\Lambda) / W_{0}( \pm \pi), \\
& \overparen{S t U}(\Lambda)^{\prime}=\overparen{S t U}(\Lambda) / h\left(W_{0}( \pm \pi)\right), \\
& E(\pi)=( \pm \pi) E(\Lambda), \\
& \widetilde{E U}(\pi)=h( \pm \pi) \widetilde{E U}(\Lambda), \\
& \widetilde{S U}(\pi)=h( \pm \pi) \widetilde{S U}(\Lambda) .
\end{aligned}
$$

Note that $( \pm \pi)$ acts on $E(\Lambda), \widetilde{E U}(\Lambda), \widetilde{S U}(\Lambda)$ by conjugation which we denote by $\alpha$. Moreover, each of these groups contains $[\pi, \pi]$. We have

$$
\begin{aligned}
& E(\pi)=\left\{( \pm \pi) \times_{\alpha} E(\Lambda)\right\} /[\pi, \pi], \\
& \widetilde{E U}(\pi)=\left\{( \pm \pi) \times{ }_{\alpha} \widetilde{E U}(\Lambda)\right\} /[\pi, \pi], \\
& \widetilde{S U}(\pi)=\left\{( \pm \pi) \times_{\alpha} \widetilde{S U}(\Lambda)\right\} /[\pi, \pi] .
\end{aligned}
$$

where, in each case, the inclusion of $[\pi, \pi]$ into the semi-direct product is the diagonal inclusion. $\alpha$ induces a unique automorphism

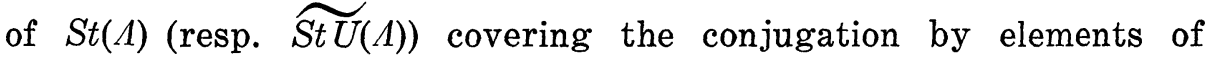
$( \pm \pi)$ in $E(\Lambda)$ (resp. $\widetilde{E U}(\Lambda))$. We define

$$
\begin{aligned}
& S t(\pi)=\left\{( \pm \pi) \times_{\alpha} \operatorname{St}(\Lambda)^{\prime}\right\} /[\pi, \pi], \\
& \widehat{S t U}(\pi)=\left\{( \pm \pi) \times_{\alpha} \overparen{\operatorname{StU}(\Lambda)^{\prime}}\right\} /[\pi, \pi] .
\end{aligned}
$$

Here, the inclusion of $[\pi, \pi]$ into the semi-direct product is the diagonal inclusion where $[\pi, \pi] \rightarrow S t(\Lambda)^{\prime}$ is induced by sending $[g, h]$ to $w_{12}(g) w_{21}(h) w_{12}(1) w_{21}(h g)$. Similarly, we have the inclusion $[\pi, \pi] \rightarrow$ $\widetilde{S t U}(\Lambda)^{\prime}$.

Let us now proceed to define abelian groups ${ }^{4} L_{n}^{s t}(\pi, w)$, which are Hermitian analogues of $W h_{2}(\pi)$. These groups are periodic of period 4 , and the definition depends on the parity of $n$.

First assume $n=2 k+1$. Let $\widetilde{S U}(\pi)$ be the group defined above with $\varepsilon=(-1)^{k}$.

Let $\mathscr{P}$ be the pullback in the diagram

\footnotetext{
${ }^{3} h$ is called the hyperbolic functor and $k$ is called the forgetting functor.

${ }^{4}$ Cf. [5].
} 


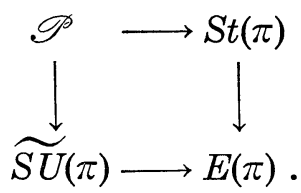

We can lift $\sigma$ to $\mathscr{P}$ and denote it by $\sigma$ again $\left(\sigma=\left(\begin{array}{ll}0 & 1 \\ \varepsilon & 0\end{array}\right)\right.$. $)$ We define $L_{n}^{s t}(\pi, w)=\mathscr{P} /([\mathscr{P}, \mathscr{P}],\{\sigma\})$ where $([\mathscr{P}, \mathscr{P}],\{\sigma\})$ denotes the subgroup of $\mathscr{P}$ generated by the commutator subgroup of $\mathscr{P}$ and $\sigma$.

Let us now assume $n=2 k$. Let $\overparen{S t U}(\pi)$ be the group defined above with $\varepsilon=(-1)^{k-1}$. We define $L_{n}^{s t}(\pi, w)=\operatorname{Ker}(\widetilde{\operatorname{St} U}(\pi) \rightarrow \widetilde{E U}(\pi))$.

THEOREM 1.1. There exists an exact sequence

$$
\begin{gathered}
L_{n+3}(\pi, w) \stackrel{\delta}{\longrightarrow} W h_{2}(\pi) /\{c+\varepsilon \bar{c}\} \stackrel{h}{\longrightarrow} L_{n}^{s t}(\pi, w) \\
\stackrel{p}{\longrightarrow} L_{n+2}(\pi, w) \longrightarrow 0
\end{gathered}
$$

where $\varepsilon=(-1)^{n}$.

Proof. Since the definition of $L_{n}^{s t}(\pi, w)$ depends on the parity of $n$, our proof is divided accordingly into two cases.

Case I. $n=2 k$. For this case, the above exact sequence is essentially the last four terms of the 'periodicity sequence' in Theorem 7.1 of [11]. We shall refer to [11] for the definitions of the homomorphisms and the proof of exactness.

Case II. $n=2 k+1$. For this case, the above exact sequence is related to, but different from the first four terms of the same 'periodicity sequence'. We shall define the homomorphisms and prove the exactness.

Definition of $\delta$. For an element $q \in L_{n+3}(\pi, w)$, it can be lifted back to an element $\hat{q} \in L_{n+1}^{s t}(\pi, w)$ by our theorem for the even case. $\hat{q}$ is representable by an element $z \in K U_{2}^{\eta}(\Lambda)$ with $\eta=(-1)^{k}$. We define $\delta(q)$ to be the element in $W h_{2}(\pi) /\{c-\bar{c}\}$ represented by $k(z)$ where $k: K U_{2}^{\eta}(\Lambda) \rightarrow K_{2}(\Lambda)$ is the natural homomorphism in (16). It is easy to see that this definition is independent of the choice of $\hat{q}$ and $z$. Alternatively, we can also define $\delta$ more directly as follows. Represent $q$ by a simple form $Q$ and view it as a matrix. (See [11] for the definition of simple form.) Lifting $Q$ to an element $\hat{Q}$ in $\operatorname{St}(\pi), \delta(q)$ is represented by $\hat{Q}\left((-1)^{k} \hat{Q}^{*}\right)^{-1}$ in $W h_{2}(\pi) /\{c-\bar{c}\}$. One may check that it is well-defined and coincides with the first definition. 
DeFinition of $h$. Represent $e \in W h_{2}(\pi) /\{c-\bar{c}\}$ by an element $z \in S t(\pi)$. Then, $h(c)$ is represented by $(z, 1) \in \mathscr{P}$.

Definition of $p$. Let $a \in L_{n}^{s t}(\pi, w)$ be represented by $(x, y) \in \mathscr{P}$. Then $p(a)$ is represented by $y \in \widetilde{S U}(\pi)$.

It is clear that $h$ and $p$ are well-defined. It follows from [11] that $\operatorname{Im}(\widetilde{S t U}(\pi) \rightarrow \mathscr{P})=[\mathscr{P}, \mathscr{P}]$. Then, the exactness at $W h_{2}(\pi) /\{c-\bar{c}\}$ and $L_{n}^{s t}(\pi, w)$ follows from that of the 'periodicity sequence.' That $p$ is an epimorphism follows from the definition.

Let $\pi_{2}$ be a $\pi$-module and let $w_{2}: \pi_{2} \rightarrow\{0,1\}=Z_{2}$ be a homomorphism of abelian groups. Let us view $Z_{2}$ as a trivial $\pi$-module. Set $\Gamma=\mathbf{Z}_{2} \oplus \pi_{2}=\mathbf{Z}_{2} \times \pi_{2}$ and $\Gamma[\pi]$ the additive group consisting of finite formal sums $\Sigma \alpha_{i} \sigma_{i}$ with $\alpha_{i} \in \Gamma$ and $\sigma_{i} \in \pi$ with component-wise addition. Following [4], we define

$$
W h_{1}(\pi ; \Gamma)=\Gamma[\pi] /\left\{\alpha \sigma-\alpha^{\tau} \tau \sigma \tau^{-1}, \beta \cdot 1\right\}
$$

where $\alpha, \beta \in \Gamma$ and $\tau, \sigma \in \pi\left(\alpha^{\tau}\right.$ means the element in $\Gamma$ gotten from $\alpha$ by the action of $\tau \in \pi)$. Again, we have an involution

$$
-: W h_{1}(\pi ; \Gamma) \longrightarrow W h_{1}(\pi ; \Gamma)
$$

defined as follows.

$$
\overline{\left(\alpha_{1}, \alpha_{2}\right) \sigma}=\left(\alpha_{1}+w_{2}\left(\alpha_{2}\right),-w_{1}(\sigma) \alpha_{2}^{\sigma^{-1}}\right) \sigma^{-1}
$$

for $\alpha_{1} \in\{0,1\}=Z_{2}, \alpha_{2} \in \pi_{2}$ and $\sigma \in \pi$.

Let us trivially modify Theorem 1.1 to

THEOREM 1.2. There is an exact sequence

$$
\begin{aligned}
L_{n+3}(\pi, w) & \longrightarrow\left\{W h_{2}(\pi) \oplus W h_{1}(\pi ; \Gamma)\right\} /\{c+\varepsilon \bar{c}\} \\
& \stackrel{h \oplus i d}{\longrightarrow} L_{n}^{s t}(\pi, w) \oplus W h_{1}(\pi ; \Gamma) /\{c+\varepsilon \bar{c}\} \stackrel{p}{\longrightarrow} L_{n+2}(\pi, w) \longrightarrow 0
\end{aligned}
$$

where $\varepsilon=(-1)^{n}$.

II. Statement of results. Let $M$ be a smooth closed $n$-dim manifold $(n \geqq 6)$. Then we can define $W h_{2}(\pi), L_{n}^{s t}(\pi, w), W h_{1}(\pi ; \Gamma)$ (where $\Gamma=Z_{2} \oplus \pi_{2}$ ) and the involution '-' of $\S I$ by taking $\pi=$ $\pi_{1} M, w: \pi \rightarrow\{ \pm 1\}$ the first Stiefel-Whitney class. $\pi_{2}=$ the $\pi$-module $\pi_{2} M$ and $w_{2}: \pi_{2} \rightarrow\{0,1\}$ the second Stiefel-Whitney class.

The main result of the paper is to identify the exact sequence (8), of the introduction, with that of Theorem 1.2. 
$H^{3}\left(\pi_{1} M ; \pi_{2} M\right)$ vanishes. Then, there is a natural isomorphism of exact sequences:

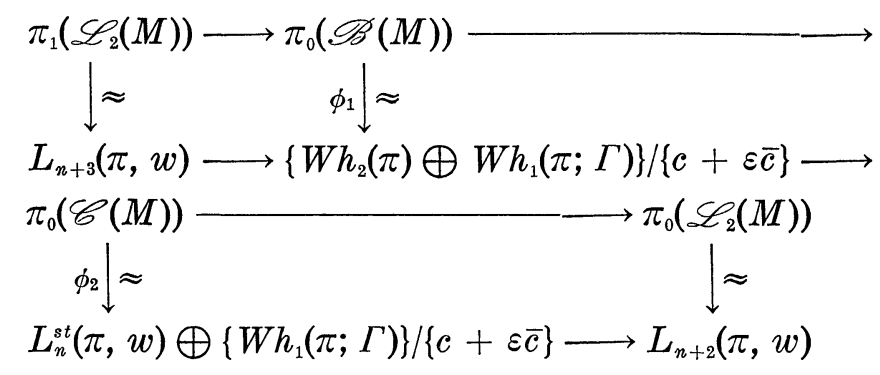

where $\varepsilon=(-1)^{n}$.

REMARK. In the argument of the well-definedness of the $\pi_{2}$-component of the invariant in $W h_{1}\left(\pi_{1} M ; Z_{2} \oplus \pi_{2} M\right)$ of [4], the author implicitly assumed that $k \in H^{3}\left(\pi_{1} M ; \pi_{2} M\right)$ vanishes. We need this assumption too. However, it has no effect on the $Z_{2}$-component.

In $\S$ III we describe the local structure of singularities of maps into a disc of $\operatorname{dim} \leqq 3$. The following sections are devoted to a proof of Theorem 2.1. Following [2] [3] [4], the maps $\phi_{1}$ and $\phi_{2}$ are defined. Once this is done, [3] applies to show that $\phi_{1}$ is an isomorphism, and the result then follows by the five-lemma.

The remainder of this section is devoted to the derivation of some simple consequences of Theorem 2.1.

Proposition 2.2. If $M$ is a smooth closed n-dim manifold $(n \geqq 6)$ satisfying the hypothesis of Theorem 2.1 and $\pi_{1} M$ contains infinitely many conjugacy classes distinct from their inverse classes, then

(A) If $\pi_{1}$ (Aut $\left.M\right) \otimes Z_{2}$ is of finite rank, then $\pi_{0}$ (Diff $M$ ) contains a subgroup isomorphic to a direct sum of infinitely many copies of $\mathrm{Z}_{2}$. (Note that we do not need to assume $k \in H^{3}\left(\pi_{1} M ; \pi_{2} M\right.$ ) equal to 0 here.)

(B) If $\pi_{1}$ (Aut $\left.M\right) \otimes Q$ is of finite rank, and $\pi_{2} M \otimes_{\pi_{1} M} Q \neq 0$, then $\pi_{0}($ Diff $M)$ contains a free abelian subgroup of infinite rank.

Proof. (A) Since $\pi$ contains infinitely many conjugacy classes distinct from their inverse classes, one can see that $W h_{1}(\pi ; \Gamma) /\{c+\varepsilon \bar{c}\}$ has $W h_{1}\left(\pi ; Z_{2}\right) /\{c+\varepsilon \bar{c}\}=\bigoplus_{1}^{\infty} Z_{2}$ as a direct summand. Since $\left[\Sigma^{i} M^{+}\right.$, $G / 0]$ are finitely generated abelian groups $(i=1,2)$ the braid (7) implies that $\pi_{0}(\mathbb{S}(M))$ contains $\bigoplus_{1}^{\infty} Z_{2}$. Then the assumption of (A) together with the sequence (10) shows that $\pi_{0}($ Diff $M) \supset \bigoplus_{1}^{\infty} Z_{2}$.

(B) Here the assumption yields a homomorphism $W h_{1}(\pi ; \Gamma) /$ 
$\{c+\varepsilon \bar{c}\} \rightarrow W h_{1}\left(\pi ; \pi_{2} \otimes_{\pi_{1}} Q\right) /\{c+\varepsilon \bar{c}\}=\bigoplus_{1}^{\infty} Q$, such that the image contains a basis. The proof then proceeds as in (A).

The following gives some control over $\pi_{1}($ Aut $M)$.

Lemma 2.3. If $M=M_{1} \times M_{2}$ is a compact manifold where $M_{1}$ is a compact $K(\pi, 1)$ and $M_{2}$ is compact space with finite fundamental group, then $\pi_{1}$ (Aut $\left.(M)\right)$ is generated by a torsion-free subgroup of finite rank (possibly infinitely generated) and a finitely generated subgroup.

Proof. By obstruction theory, we know that $\pi_{1}\left(\right.$ Aut $\left.M_{1}\right) \approx$ Centre $\pi$ and thus it is a torsion-free subgroup of finite rank, i.e., a subgroup of $\bigoplus_{1}^{l} Q$ for some $0 \leqq l<\infty$. Let $f: S^{1} \times M_{1} \times M_{2} \rightarrow M_{1} \times M_{2}$ represent an element of $\pi_{1}$ (Aut $M$ ). Since $\pi$ is torsion-free, the induced $\operatorname{map} \pi_{1}\left(M_{2}\right) \rightarrow \pi_{1}\left(M_{1}\right)$ is trivial, so $f(t, x, y)=(h(t, y), g(t, x, y))$. Composing with an element of $\pi_{1}$ (Aut $M_{1}$ ) (i.e., the inverse of $h$ ), we can assume that $f(t, x, y)=(x, g(t, x, y))$ where $g$ is a unit in the monoid $\left[\left(S^{1}, 1\right) \times M_{1},\left(M_{2}^{M_{2}}\right.\right.$, id $\left.)\right]$. Since $S^{1} \times M_{1}, M_{2}$ are finite $C W$ complexes and $\pi_{1} M_{2}$ is finite, it follows from the standard obstruction theory that the group of units is finitely generated.

EXAmPle 1. Let $M$ be a smooth closed $K(\pi, 1)$ such that $\pi$ has infinitely many conjugacy classes distinct from their inverse classes (e.g., $M=T^{n}$, a compact solvmanifold, $X / \Gamma$ where $X$ is a noncompact symmetric space and $\Gamma$ a torsion-free uniform lattice, a 3-dim manifold with sufficiently large fundamental group etc.). Let $N$ be a smooth closed manifold with finite fundamental group. If dim $(M \times N) \geqq 6$, then the hypothesis (A) is verified so that $\pi_{0}(\operatorname{Diff}(M \times N))$ contains a subgroup isomorphic to $\bigoplus_{1}^{\infty} \mathbf{Z}_{2}$.

ExAmple 2. Let $M$ and $N$ be as above. Then $M \times N \times S^{2}$ verifies the hypothesis (B) if $\operatorname{dim} M \times N \times S^{2} \geqq 6$ and the first $k$-invariant of $N$ vanishes. Thus $\pi_{0}\left(\operatorname{Diff}\left(M \times N \times S^{2}\right)\right)$ contains a subgroup of infinite rank. In particular, $\pi_{0}\left(\operatorname{Diff}\left(T^{n} \times S^{2}\right)\right)(n \geqq 4)$ has infinite rank.

Now we prepare to consider some higher homotopy groups. Let $\operatorname{Diff}(W, \partial)$ be the group of diffeomorphisms of the manifold with boundary $\partial W$ which are the identity on the boundary.

Following an idea of Siebenmann's, we have the following lemma, which was also observed by A. Hatcher, and Burgelea-LashofRothenberg.

Lemma 2.4. There is a map $i: \operatorname{Diff}\left(M \times I^{k}, \partial\right) \rightarrow \Omega^{k} \operatorname{Diff}\left(M \times T^{k}\right)$ 
which has a left inverse, up to homotopy, so that $\pi_{0}\left(\operatorname{Diff}\left(M \times I^{k}, \partial\right)\right)$ is a direct summand of $\pi_{k}\left(\operatorname{Diff}\left(M \times T^{k}\right)\right)$.

Proof. We construct the map $i: \operatorname{Diff}(M \times I, \partial) \rightarrow \Omega \operatorname{Diff}\left(M \times S^{1}\right)$, and one can check that the construction can be iterated to verify the claim. We shall regard $\theta \in[0,1]$ as the element of $\operatorname{Diff}\left(M \times S^{1}\right)$ which spins the second factor by $e^{2 \pi i \theta}$. Then $i(\phi)(\theta)=[\theta, \phi]$ (the commutator). We construct a left inverse by completing the diagram

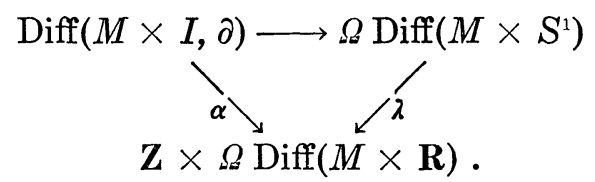

Here $\alpha(\phi)=(0,[\theta, \hat{\phi}])$ where $\hat{\phi} \in \operatorname{Diff}(M \times \mathbf{R})$ agrees with $\phi$ on $M \times I$, and is invariant under conjugation by integral translation, and $\lambda\left(\phi_{t}\right)=\left(n, \tilde{\phi}_{t}-t n\right)$ where $\tilde{\phi}_{t}: M \times \mathbf{R} \times I \rightarrow M \times \mathbf{R}$ is a lift of $\phi_{t}$ such that $\hat{\phi}_{0}=\mathrm{Id}$ and $\hat{\phi}_{1}=$ covering translation by $n$.

We have two fibrations:

$$
\begin{aligned}
& \operatorname{Diff}(M \times I, \partial) \longrightarrow \mathscr{E}(M \times I, M \times \mathbf{R} \text { rel } M \times 0) \\
& \operatorname{Diff}(M \times \mathbf{R ~ r e l ~} M \times 0) \longrightarrow \operatorname{Diff}(M \times \mathbf{R}) \stackrel{\mid M \times 1}{\stackrel{\mid M \times 0}{\longrightarrow} \mathscr{E}(M, M \times \mathbf{R})} \mathscr{E}(M, M \times \mathbf{R})
\end{aligned}
$$

where $\mathscr{E}$ denotes the space of embeddings.

Now $\mathscr{E}(M \times I, M \times \mathbf{R}$ rel $M \times 0)$ and $\operatorname{Diff}(M \times \mathbf{R}$ rel $M \times 0)$ are contractible, so $\operatorname{Diff}(M \times I, \partial) \approx \Omega \mathscr{E}(M, M \times \mathbf{R}) \approx \Omega(\operatorname{Diff} M \times \mathbf{R})$ is a homotopy equivalence. Since this composite is $\alpha$, and the diagram above commutes, we are done.

EXAMPlE 3. $\pi_{i}\left(\operatorname{Diff}\left(T^{n-2} \times S^{2}\right)\right)$ has infinite rank for $0 \leqq i \leqq n-3$, for it contains $\pi_{0}\left(\operatorname{Diff}\left(S^{2} \times T^{n-2-i} \times I^{i}, \partial\right)\right.$ as a direct summand, and since the rel $\partial$ version of our theory is the same as the closed case, this later group has infinite rank for $0 \leqq i \leqq n-3$.

\section{EXAMPLE $4 . \pi_{i}\left(\right.$ Diff $\left.T^{n}\right)$.}

The map Diff $T^{n} \rightarrow$ Aut $T^{n}$ has a left inverse, so we get the split exact sequence

$$
1 \longrightarrow \pi_{0}\left(\&\left(T^{n}\right)\right) \longrightarrow \pi_{0}\left(\operatorname{Diff} T^{n}\right) \longrightarrow G L(n, \mathbf{Z}) \longrightarrow 1
$$

To compute $\pi_{0}\left(\&\left(T^{n}\right)\right)$ we pass to the braid (7). Since $W h_{2}\left(\mathbf{Z}^{n}\right)=0$ and $L_{n+3}\left(\mathbf{Z}^{n}, w\right)$ maps to this summand of $\pi_{0}\left(\mathscr{B}\left(T^{n}\right)\right)$, we see the map is zero. Moreover, $L_{n+3}\left(\mathbf{Z}^{n}, w\right) \rightarrow \pi_{1}\left(\mathscr{S}\left(T^{n} \times\left(S^{1}, 1\right)\right)\right.$ is onto by [7], 
[13], so that $\pi_{1}\left(\mathscr{S}\left(T^{n} \times\left(S^{1}, 1\right)\right) \rightarrow \pi_{0}\left(\mathscr{B}\left(T^{n}\right)\right)\right.$ is zero. Thus, we have the short exact sequence

$$
1 \longrightarrow \pi_{0}\left(\mathscr{B}\left(T^{n}\right)\right) \longrightarrow \pi_{0}\left(\mathscr{S}\left(T^{n}\right)\right) \longrightarrow \pi_{0}\left(\mathscr{S}\left(T^{n} \times\left(S^{1}, 1\right)\right)\right) \longrightarrow 1 .
$$

We can regard $\pi_{0}\left(\mathscr{S}\left(M \times\left(S^{1}, 1\right)\right)\right)$ as the smoothings of the standard topological $M \times\left(S^{1}, 1\right)$; so it is the kernel of $\pi_{0}\left(\right.$ Diff $\left.T^{n}\right) \rightarrow \pi_{0}\left(\right.$ Homeo $\left.T^{n}\right)$, this provides a splitting for the sequence (30) and we get:

THEOREM 2.5. If $n \geqq 6$,

(A) $\pi_{0}\left(\right.$ Diff $\left.T^{n}\right) \approx\left(W h_{1}\left(\mathbf{Z}^{n} ; \mathbf{Z}_{2}\right) /\{c+\bar{c}\} \oplus \pi_{0}\left(\mathscr{S}\left(T^{n} \times\left(S^{1}, 1\right)\right)\right\} \times{ }_{\alpha} G L(n, \mathbf{Z})\right.$

(B) $\pi_{i}\left(\right.$ Diff $\left.T^{n}\right)(i<n)$ contains $\bigoplus_{1}^{\infty} \mathbf{Z}_{2}$.

REMARKS. (1) (B) follows from Lemma 2.4 since $W h_{1}\left(\mathbf{Z}^{n} ; \mathbf{Z}_{2}\right) \approx$ $\bigoplus_{1}^{\infty} \mathbf{Z}_{2}$.

( 2$) \quad \pi_{0}\left(\mathscr{S}\left(T^{n} \times\left(S^{1}, 1\right)\right)\right)=H^{2}\left(T^{n} \times\left(S^{1}, 1\right) ; \mathbf{Z}_{2}\right) \oplus\left\{\bigoplus_{i} H^{i}\left(T^{n} \times\left(S^{1}, 1\right) ; \Gamma_{i}\right)\right\}$ where $\Gamma_{i}=\pi_{i}(P D / 0)$ as computed in [7] [13].

(3) The action $\alpha$ of $G L(n, \mathbf{Z})$ is the obvious one.

(4) Note that $\pi_{0}\left(\right.$ Diff $\left.T^{n}\right)$ contains many $\mathbf{Z}_{2}$ coming from different sources.

(5) Our theory works in the PL and topological categories, in which case $\pi_{0}\left(\mathscr{S}\left(T^{n} \times\left(S^{1}, 1\right)\right)\right)$ is replaced by the corresponding groups, i.e., $H^{2}\left(T^{n} \times\left(S^{1}, 1\right) ; \mathbf{Z}_{2}\right)$ and 0 respectively.

III. Geometry of singularities. In this section, we shall discuss the singularities of maps

$$
f:\left(W^{n+s} \partial W^{n+s}\right) \longrightarrow\left(I^{s}, \partial I^{s}\right) \quad(s \leqq 3) .
$$

Much of the material is well-known [2] [3] [14], so we limit ourselves to statement of results. We follow [3] closely for the geometric description of the singularities. Throughout we assume that $f:\left(W^{n+s}, \partial W^{n+s}\right) \rightarrow\left(I^{s}, \partial I^{s}\right)$ is free of singularities near $\partial W^{n+s}$. We shall call a local coordinate system $\left(z_{0}, \cdots, z_{s-1}\right)$ for $I^{s}$ admissible if $z_{i}=\varphi_{i}\left(w_{i}, \cdots, w_{s-1}\right)$ with $\partial \varphi_{i} / \partial w_{i}>0$ where $w_{i}: I^{s} \rightarrow I$ is the standard projection to the $i$ th factor. We shall consider the $z_{0}$ direction as vertical and the idea is to think of $z_{1}, \cdots, z_{s-1}$ as parametrizing the slices $f^{-1}\left(I \times\left(z_{1}, \cdots, z_{s-1}\right)\right)$ and the functions $f \mid$ slice.

In each case $(s=1,2,3), f$ is homotopic (rel $\partial)$ by a homotopy as small as we please, to a smooth map whose singularities with respect to some properly chosen coordinate system $x_{1}, \cdots, x_{s-1}$, $y_{1}, \cdots, y_{n+1}$ on $W^{n+s}$ and an admissible coordinate system on $I^{s}$, have only a few possible forms. We shall list them and describe their geometry. 
Let $f: W^{n+s} \rightarrow I^{s}$ be a map. We define the graphic of $f$ to be $\bigcup_{z \in I^{s}}$ [critical values of $f$ ]. We define a gradient-like vector field $\xi$ of $f$ on $W^{n+s}$ (with respect to an admissible coordinate system of $\left.I^{s}\right)$ as follows:

(1) $\xi\left(z_{0} f\right) \geqq 0$ vanishing only on singularities of $f$,

(2) $\xi\left(z_{i} f\right)=0(i>0)$,

(3) Near each singular point of $f$ there exist coordinates $x_{1}, \cdots, x_{s-1}, y_{1}, \cdots, y_{n+1}$ as above, with respect to which $\xi$ is the gradient field of $z_{0} f \mid$ slice .

Gradient-like vector fields always exist and can be chosen so that, for the most part, the trajectories of their critical points are in general position. Let $p$ be a critical point in a slice and let $\varphi_{t}$ be the one parameter group of diffeomorphisms of the slice generated by the gradient-like vector field $\xi$. Define the stable and unstable set of $p$, written as $W(p)$ and $W^{*}(p)$ respectively, by the equations,

$$
\begin{gathered}
W(p)=\left\{x \mid \lim _{t \rightarrow \infty} \varphi_{t}(x)=p\right\} \\
W^{*}(p)=\left\{x \mid \lim _{t \rightarrow-\infty} \varphi_{t}(x)=p\right\} .
\end{gathered}
$$

Let us now list the generic singularities for $f: W^{n+s} \rightarrow I^{s}(n \geqq 6, s \leqq 3)$.

(A) $f: W^{n+1} \rightarrow I$

The singularities of Morse type: Given by the formula

$$
z_{0}=-y_{1}^{2}-\cdots-y_{\lambda}^{2}+y_{\lambda+1}^{2}+\cdots+y_{n+1}^{2}
$$

with origin identified as the isolated singular point $p$. The graphic is

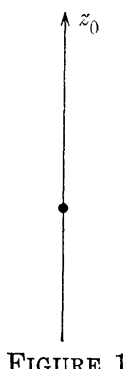

In a neighborhood of $p, W(p)=D^{\lambda}$ and $W^{*}(p)=D^{n+1-\lambda}$. This is the usual case of attaching a handle of index $\lambda$ (to the level surface $f^{-1}(c)$ for $\left.c<0\right)$. Or equivalently, we may say that we attach a dual $(n+1-\lambda)$-handle (to the level surface $f^{-1}(c)$ for $c>0$ ).

(B) $f: W^{n+2} \rightarrow I^{2}$. There are three types of singularities.

(1) A line of $\lambda$-handles with graphic 


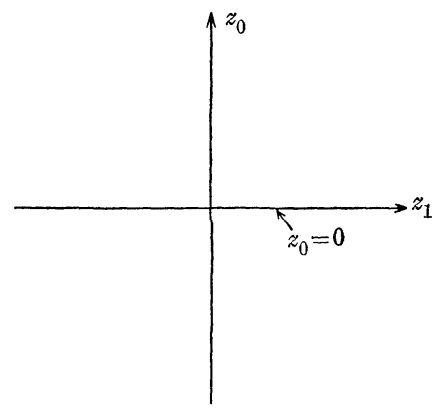

FIGURE 2

and formula

$$
\begin{gathered}
z_{0}=-y_{1}^{2}-\cdots-y_{\lambda}^{2}+y_{\lambda+1}^{2}+\cdots+y_{n+1}^{2} \\
z_{1}=x_{1} .
\end{gathered}
$$

For a vertical line $z_{1}=$ const., it is a Morse type of singularity on the corresponding slice. Therefore, in the neighborhood of the singular set, the stable and unstable sets are 1-parameter families of $\lambda$-handles and $(n+1-\lambda)$-handles respectively.

(2) A birth-death cusp giving rise to a complementary pair of lines of handles of index $\lambda$ and $\lambda+1$ with graphic

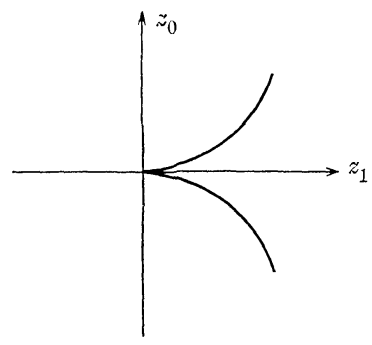

$\left(z_{0} / 2\right)^{2}=\left(z_{1} / 3\right)^{3}$

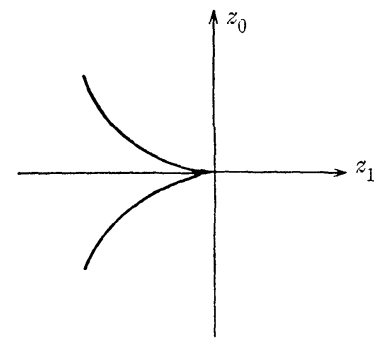

$\left(z_{0} / 2\right)^{2}=-\left(z_{1} / 3\right)^{3}$

FIGURE 3

and formula

$$
\begin{gathered}
z_{0}=x_{1} y_{1}-\left( \pm y_{1}^{3}\right)-y_{2}^{2}-\cdots-y_{\lambda+1}^{2}+y_{\lambda+2}^{2}+y_{\lambda+3}^{2}+\cdots+y_{n+1}^{2} \\
z_{1}=x_{1}
\end{gathered}
$$

Let us examine the case of $z_{0}=x_{1} y_{1}-y_{1}^{3}-\cdots-y_{\lambda+1}^{2}+\cdots+y_{n+1}^{2}$ corresponding to the graphic on the left of Figure 3 more carefully. The other one is similar. When $x_{1}<0$, there is no critical point on the corresponding slice. When $x_{1}>0$, we have two non-degenerate critical points of index $\lambda$ and $\lambda+1$ respectively. At $x_{1}=0$, it is 
Whitney's folding singularity [14] with the cusp as the graphic. Let us describe the stable and unstable sets near the folding singularity. At $x_{1}=0, W(0)$ and $W^{*}(0)$ are half discs of $\operatorname{dim}(\lambda+1)$ and $(n-\lambda+1)$ respectively. When we move slightly to the right (i.e., $0<x_{1}<\varepsilon$ ), the unstable set of the lower critical point (which is the dual $(n-\lambda+1)$-handle) meets the stable set of the upper critical point (which is a $(\lambda+1)$-handle) transversely at a single point in a level surface between the critical points, e.g., $f^{-1}\left(0, z_{1}\right)$.

(3) A surgery birth-death point with vertical tangent giving rise to a dual pair of lines of handles of index $\lambda$ and $n+1-\lambda$ with graphic

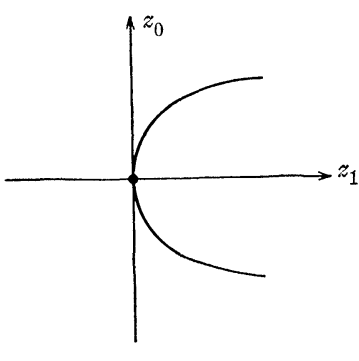

$z_{1}=z_{j}^{2}$

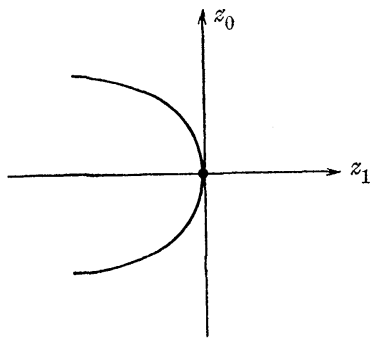

$z_{1}=-z_{0}^{2}$

FIgURE 4

and formula

$$
\begin{gathered}
z_{0}=x_{1} \\
z_{1}= \pm x_{1}^{2}-y_{1}^{2}-\cdots-y_{\lambda}^{2}+y_{\lambda+1}^{2}+\cdots+y_{n+1}^{2}
\end{gathered}
$$

This is actually isomorphic to (1) - a line of $\lambda$-handles if we turn $z_{0}, z_{1}$ around. $z_{1} f$ has one critical point of Morse type with index given in the box. We can give the explicit gradient-like vector field by

$$
\xi=\left(y_{1}^{2}+\cdots+y_{n+1}^{2}, \pm x_{1} y_{1}, \cdots, \pm x_{1} y_{\lambda}, \mp x_{1} y_{\lambda+1}, \cdots, \mp x_{1} y_{n+1}\right) \text {. }
$$

Let us describe the geometry of this case with some care. We examine the case corresponding to the graphic on the left. The other case is similar. For the slice corresponding to $z_{1}<0$, there is no critical point. For a slice corresponding to $0<z_{1}<\varepsilon$, the unstable set of the lower critical point forms a dual $(n+1-\lambda)$ handle while the stable set of the higher critical point forms an $(n+1-\lambda)$-handle. They meet on their boundaries at the level 
surface $f^{-1}\left(0, z_{1}\right)$ to form an $(n+1-\lambda)$-sphere. In other words, the ascending trajectories issueing from the lower critical point and the descending trajection issueing from the higher critical point coincide and form a sphere of $\operatorname{dim}(n+1-\lambda)$. When we move the slice to the left, the size of the sphere diminishes and the pair of critical points is eventually eliminated. The slice corresponding to $0<z_{1}<\varepsilon$ is gotten from the slice corresponding to $z_{1}<0$ by a surgery on a sphere of $\operatorname{dim} \lambda-1$.

Of course, when we come to a slice corresponding to $z_{1}>\varepsilon$, we can perturb the gradient-like vector field $\xi$ slightly such that the unstable set of the lower critical point and the stable set of the higher critical point meet in general position. But we can not make them in general position near the slice corresponding to $z_{1}=0$. This is what we call a surgery birth-death point.

(C) $f: W^{n+3} \rightarrow I^{3}$. There are seven types of generic singularities.

(1) A sheet of $\lambda$-handles with graphic

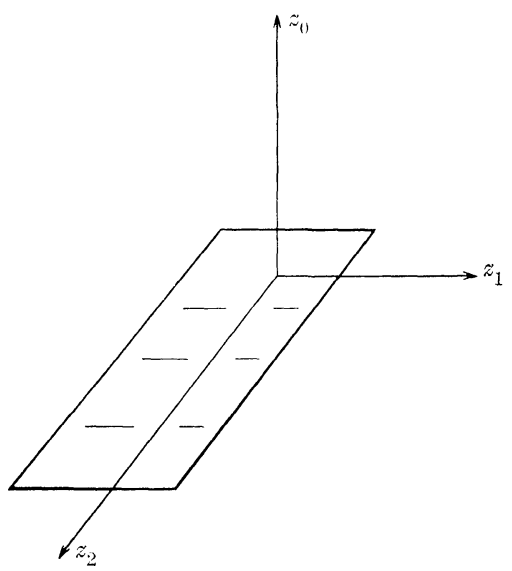

Figure 5

and formula

$$
\begin{aligned}
& z_{0}=-y_{1}^{2}-\cdots-y_{\lambda}^{2}+y_{i+1}^{2}+\cdots+y_{n+1}^{2} \\
& z_{1}=x_{1} \\
& z_{2}=x_{2}
\end{aligned}
$$

This is just the 2-dim correspondent of a line of $\lambda$-handles.

(2) A line of cusps giving rise to a complementary pair of sheets of handles of index $\lambda$ and $\lambda+1$ with graphic 

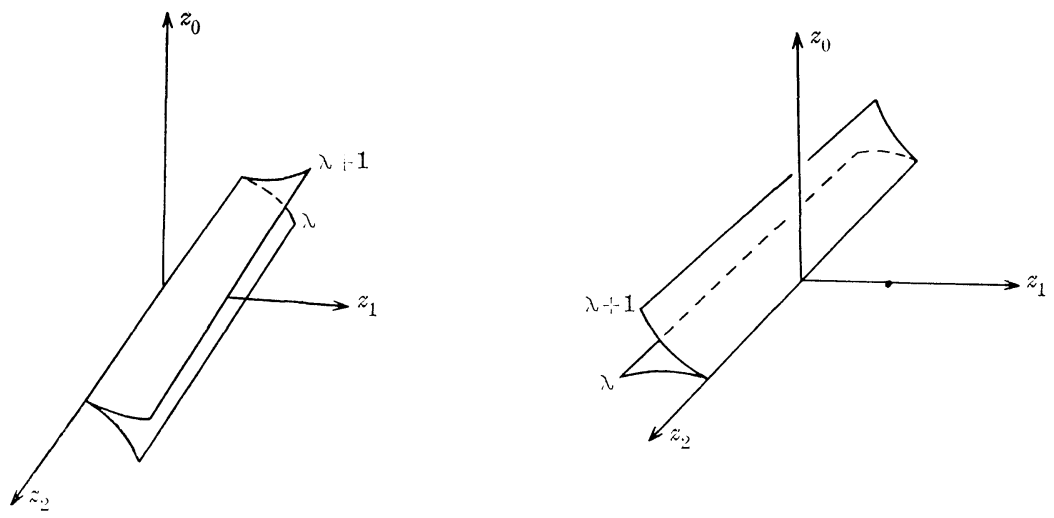

FiguRe 6

and formula

$$
\begin{aligned}
& z_{0}=x_{1} y_{1}-\left( \pm y_{1}^{3}\right)-y_{2}^{2}-\cdots-y_{\lambda+1}^{2}+y_{\lambda+2}^{2}+\cdots+y_{n+1}^{2} \\
& z_{1}=x_{1} \\
& z_{2}=x_{2}
\end{aligned}
$$

For $z_{2}=a, f \mid W(a)=f^{-1}\left(z_{0}, z_{1}, a\right)$ is previously described as birthdeath cusp. The geometry is unchanged, when we change the plane $z_{2}=$ const.

(3) A line of surgery birth-death points giving rise to a pair of sheets of handles of index $\lambda$ and $n+1-\lambda$ with graphic
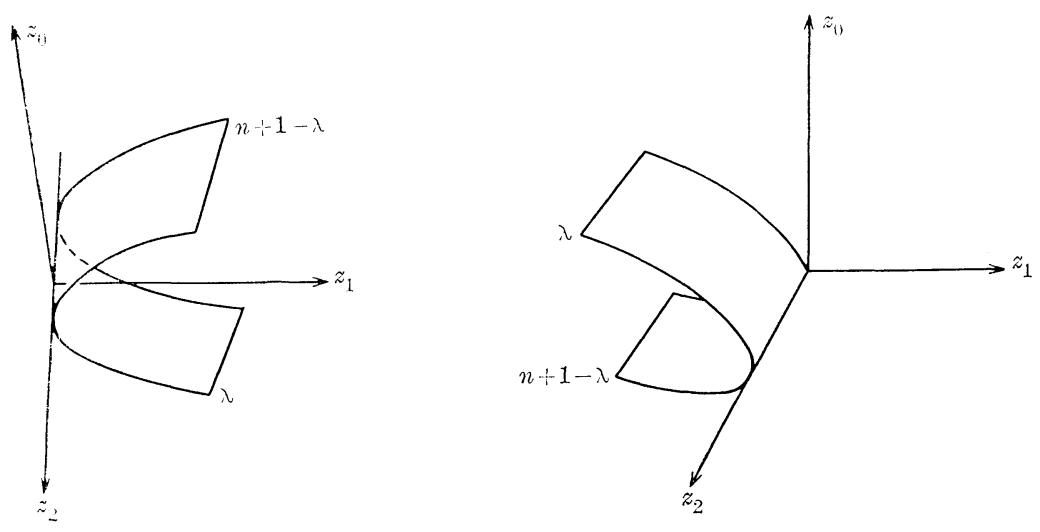

Figure 7

and formula

$$
\begin{aligned}
& z_{0}=x_{1} \\
& z_{1}= \pm x_{1}^{2}-y_{1}^{2}-\cdots-y_{\lambda}^{2}+y_{\lambda+1}^{2}+\cdots+y_{n+1}^{2} \\
& z_{2}=x_{2}
\end{aligned}
$$


This is the corresponding case for surgery birth-death points as that for cusps in (2).

(4) A curve of cusps tangent to the $\left(z_{0}, z_{1}\right)$ - plane with graphic
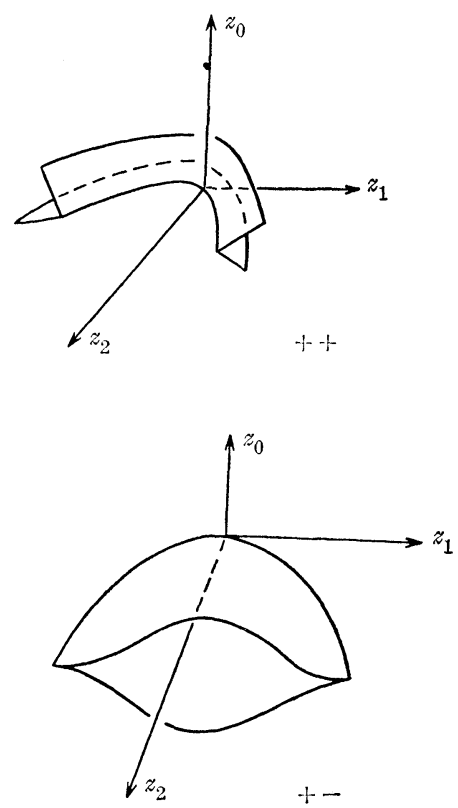
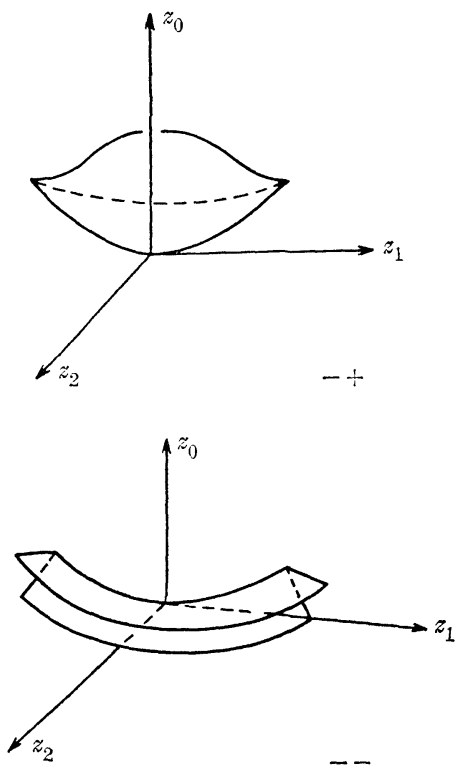

FIGURE 8

and formula

$$
\begin{aligned}
& z_{0}=x_{1} y_{1} \pm y_{1}^{3}-y_{2}^{2}-\cdots-y_{\lambda}^{2}+y_{\lambda+1}^{2}+\cdots+y_{n+1}^{2} \\
& z_{1}=x_{2} \\
& z_{2}=x_{1} \pm x_{2}^{2} .
\end{aligned}
$$

For $z_{1}=a, f \mid W(a)=f^{-1}\left(z_{0}, a, z_{2}\right)$ is previously described as the birthdeath cusp. For $z_{2}=b>0$ in the case of the left hand upper corner, $f \mid W(b)=f^{-1}\left(z_{0}, z_{1}, b\right)$ consists of one birth cusp and one death cusp of index $\lambda$ and $\lambda+1$; when $z_{2} \rightarrow 0$, these two cusps cancel each other and become one line of handles of index $\lambda$ and another line of handles of index $\lambda+1$ for $z_{2}=b<0$. For the left hand lower corner case, cusps and lines of handles all cancel out when $z_{2}$ moves from $>0$ to $<0$. The other two cases are similar.

(5) A surgery birth-death tangent to the $\left(z_{0}, z_{1}\right)$ - plane giving rise to a dual pair of sheets of handles of index $\lambda$ and $n+1-\lambda$ with graphic 

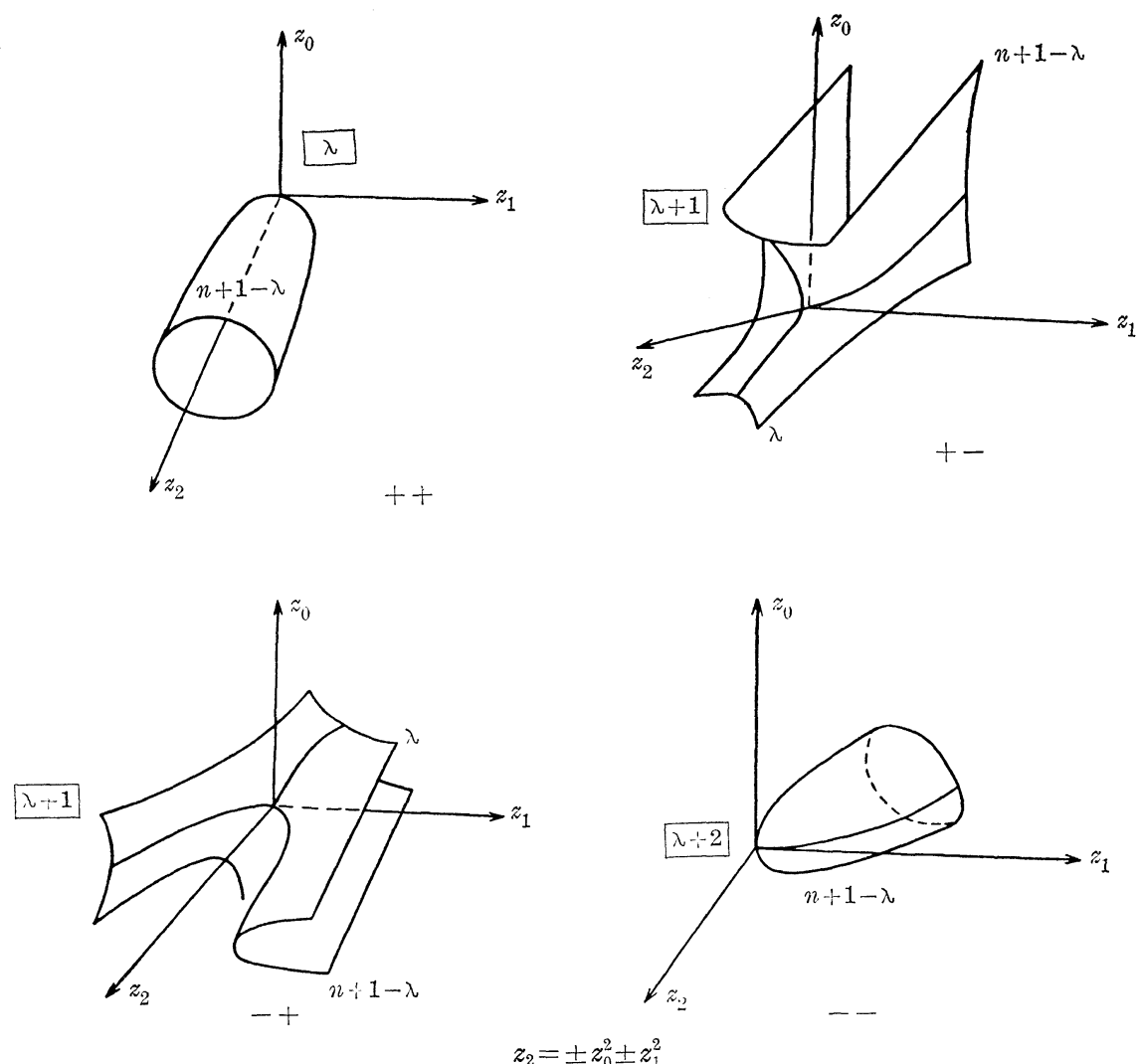

FIGURE 9

and the formula

$$
\begin{aligned}
& z_{0}=x_{1} \\
& z_{1}=x_{2} \\
& z_{2}= \pm x_{1}^{2} \pm x_{2}^{2}-y_{1}^{2}-\cdots-y_{\lambda}^{2}+y_{\lambda+1}^{2}+\cdots+y_{n+1}^{2} .
\end{aligned}
$$

Let us describe the geometry for the cases of the left column. The cases corresponding to the right column are similar but in reverse. First, we consider the case of the left upper corner. For $z_{2}=b>0$, $f \mid W(b)=f^{-1}\left(z_{0}, z_{1}, b\right)$ consists of one surgery birth and one surgery death. As we pointed out before, the trajectories come down from the critical point of index $n+1-\lambda$ and the trajectories go up from the critical point of index $\lambda$ form a sphere of $\operatorname{dim} n+1-\lambda$ if we are near the surgery birth-death point. This sphere gradually shrinks its size until it altogether vanishes when it passes the surgery birth-death point. At the present situation, we have two such spheres and they move toward each other and eventually become identified instead of moving toward some surgery birth-death 
points. This is true at least for $\varepsilon>b>0$. In fact, these spheres which correspond to different values of $z_{1}$ together with the two surgery birth-death points form a sphere of $\operatorname{dim} n+2-\lambda$. The size of the sphere diminishes as $b \rightarrow 0$ and vanishes for $b<0$. The function $z_{2} f$ is of Morse type and it has one critical point of index $\lambda$.

Next, we consider the left lower corner case. For $z_{2}=b>0$, $f \mid W(b)=f^{-1}\left(z_{0}, z_{1}, b\right)$ has one surgery death point and one surgery birth point. These two points come close to cancel themselves out. Therefore, when $b<0$, the spheres which are supposedly vanished after passing the surgery birth-death points just move on and no longer vanish since the surgery birth and death points have already cancelled themselves out. The function $z_{2} f$ has one critical point of Morse type. The index of this critical point is $\lambda+1$.

(6) A swallow's tail giving rise to two lines of cusps with
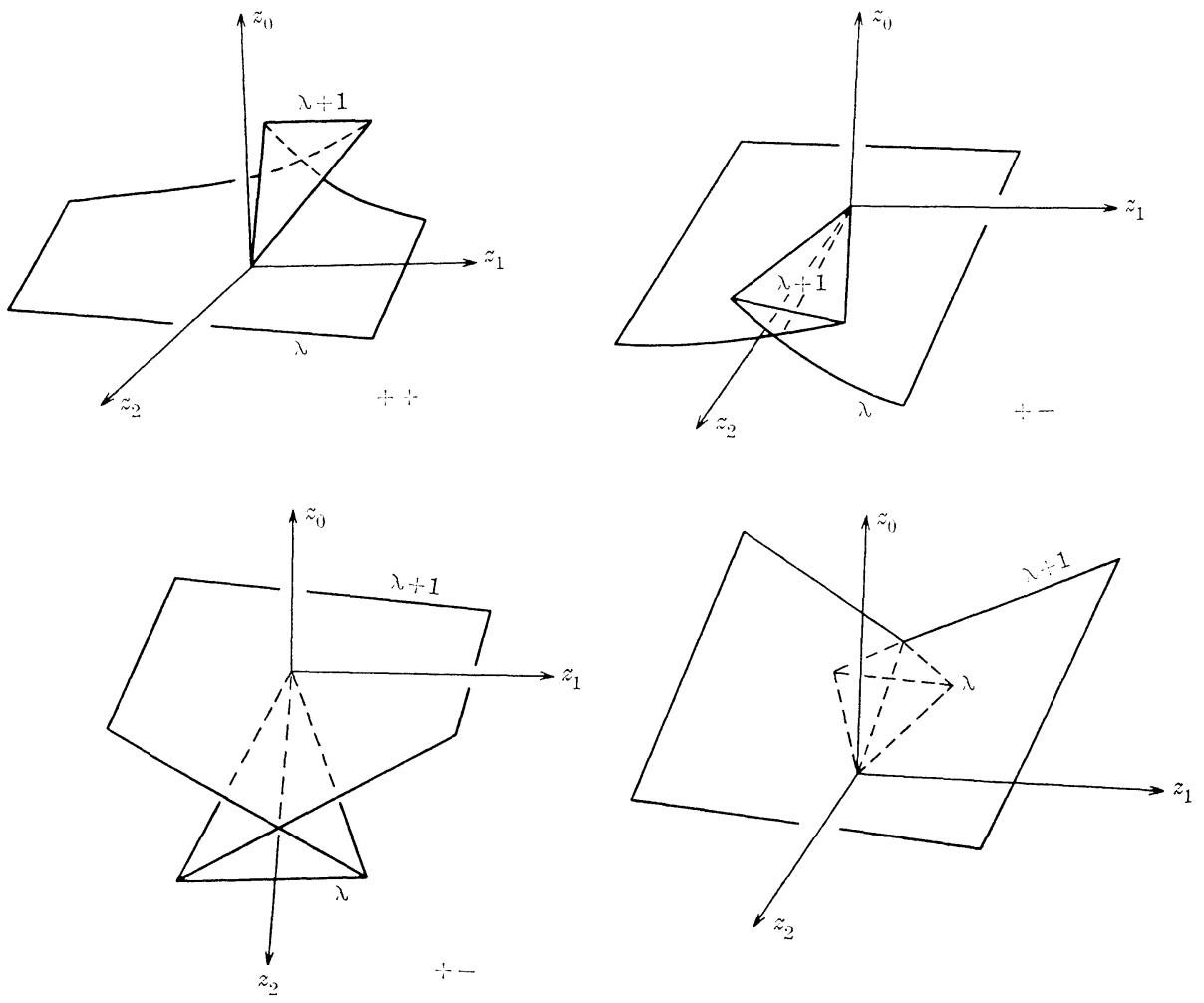

FIGURE 10

graphic and formula

$$
\begin{aligned}
& z_{0}= \pm x_{1} y_{1} \pm x_{2} y_{1}^{2}+y_{1}^{4}-y_{2}^{2}-\cdots-y_{\lambda+1}^{2}+y_{\lambda+2}^{2}+\cdots+y_{n+1}^{2} \\
& z_{1}=x_{1} \\
& z_{2}=x_{2} .
\end{aligned}
$$


Let us describe the geometry for the case of the right upper corner. The other cases are similar. For $z_{2}=b>0, f \mid W(b)=f^{-1}\left(z_{0}, z_{1}, b\right)$ consists of one birth cusp and one death cusp. We consider the slice corresponds to $z_{1}=$ const. and describe the change when $z_{1}$ moves from the left to the right. At the far left, it has only one nondegenerate critical point (of Morse type) of index $\lambda$. After we pass the birth cusp, we obtain a pair of nondegenerate critical points of index $\lambda$ and $\lambda+1$. The descending $(\lambda+1)$-handle (i.e. the stable set) of the newly created critical point of the higher index first meets the dual ascending $(n+1-\lambda)$-handle (i.e., the unstable set) of the newly created critical point of lower index at a single point transversely in a level surface between them, then it comes down to meet the dual ascending $(n+1-\lambda)$-handle of the old critical point of index $\lambda$ again at a single point transversely in a lower level surface (above the old critical point). When we move to the right, the rôle of the two critical points of lower index are interchanges. Finally, it becomes a critical point of index $\lambda$ again when we move to the far right. All of this happens in a disc in $W(b)=F^{-1}\left(z_{0}, z_{1}, b\right)$. When $b \rightarrow 0$, the size of the disc supporting this phenomenon diminishes and eventually vanishes. After we pass $b=0, W(b)$ just has a line of $\lambda$-handles for $b>0$.

(7) The resolution of cusps with graphic
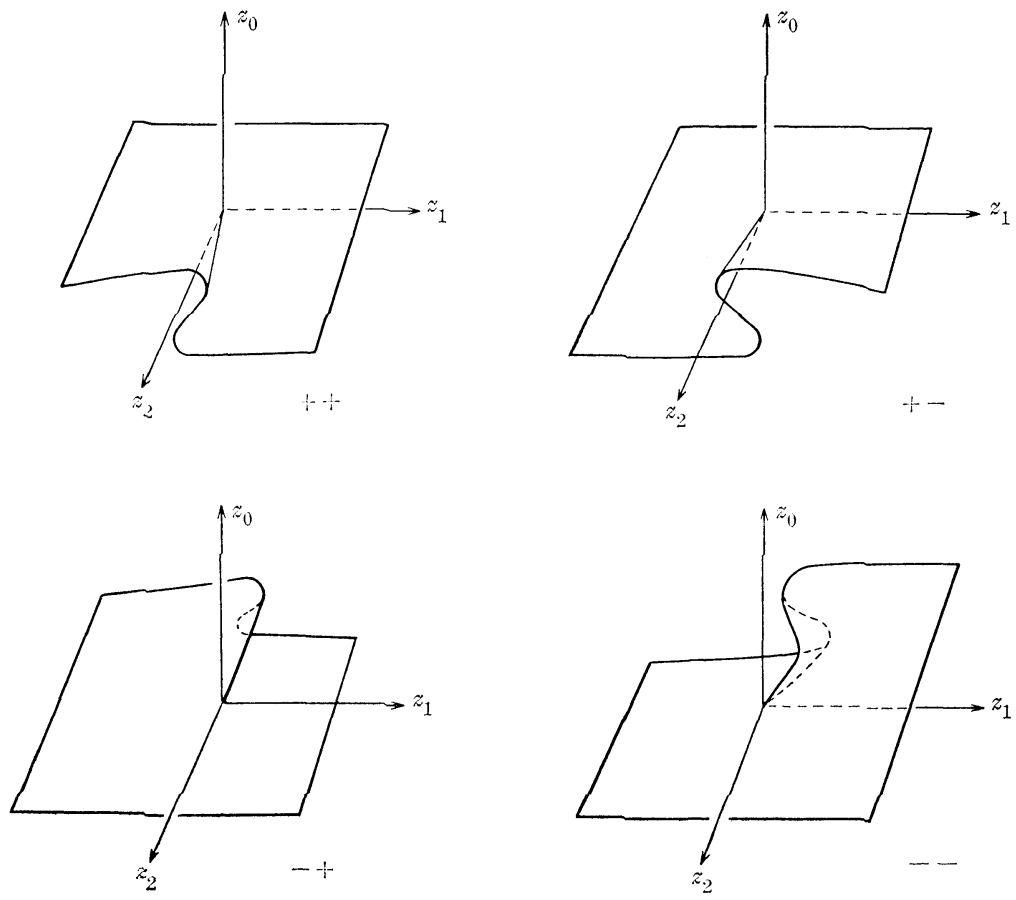

$z_{1}^{2}=-\left( \pm z_{0}^{2}\right) \pm z_{0} z_{2}$

FIGURE 11 
and formula

$$
\begin{aligned}
& z_{0}=y_{1} \\
& z_{1}=x_{1} y_{1} \pm y_{1}^{3}-y_{2}^{2}-\cdots-y_{\lambda+1}^{2}+y_{\lambda+2}^{2}+\cdots+y_{n+1}^{2} \\
& z_{2}= \pm x_{1}
\end{aligned}
$$

This is the corresponding case for surgery birth-death points as that for cusps in (6). Again, we shall describe the left upper corner case only. For $z_{2}=b>0, f \mid W(b)=f^{-1}\left(z_{0}, z_{1}, b\right)$ has one surgery birth and one surgery death. The spheres made of trajectories are of $\operatorname{dim}(n+1-\lambda)$ and $\lambda$ respectively. They meet at the middle line of critical points. The spheres of trajectories above the middle line of critical points together with the surgery death form a disc of $\operatorname{dim} \lambda+1$. Similarly, the spheres of trajectories under the middle line of critical points form a disc of $\operatorname{dim} n+2-\lambda$. These two discs have the middle line of critical points in common and live in a disc. When $b \rightarrow 0$, the size of the discs diminishes and eventually vanishes as we pass $b=0$. For $b<0$, we just have a line of $\lambda$-handles. Note that if we follow by projection on the $\left(z_{1}, z_{2}\right)$-plane, we get the map $W^{n+3} \rightarrow I^{2}$ of birth death cusp.

IV. Cobordism representation of $\pi_{i}(\mathscr{B}(M))$ and $\pi_{i}(\mathscr{C}(M))$. In this section, we shall give cobordism representations of $\pi_{i}(\mathscr{B}(M))$ and $\pi_{i}(\mathscr{C}(M))$. Let us first describe $\pi_{i}(\mathscr{C}(M))$.

Consider an object $\alpha=(W(\alpha), f)$

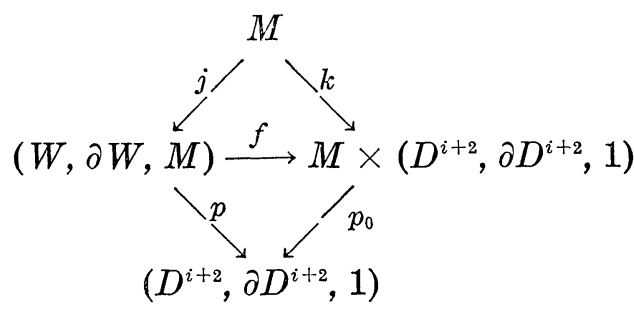

satisfying the following conditions:

(1) 1 is the base point of $\partial D^{2+2}$ and the upper triangle is commutative with $j$ a diffeomorphism and $k$ the standard identification of $M$ with $M \times 1$,

(2) $f$ is a normal map [13],

(3) the lower triangle is commutative with $p_{0}$ the projection onto the second factor, and $M \stackrel{j}{\longrightarrow} \partial W \stackrel{p}{\longrightarrow} \partial D^{i+2}$ is a smooth fibration.

Two such objects $\alpha_{1}, \alpha_{2}$ are cobordant if we have a normal cobordism 


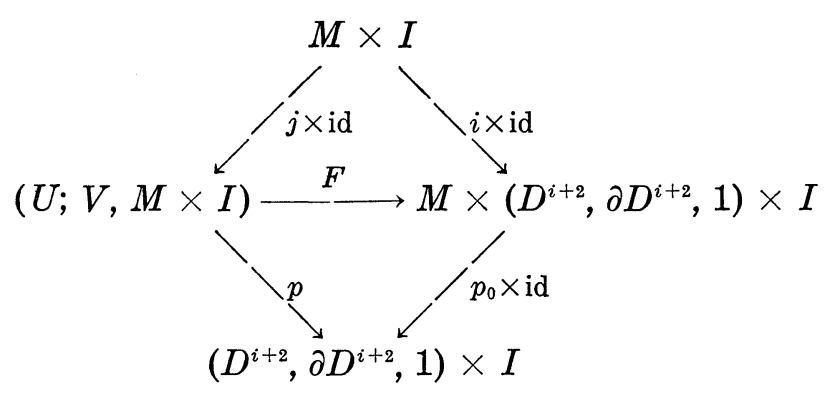

satisfying the following conditions:

(1) The upper triangle is commutative with $U$ a normal cobordism between $W\left(\alpha_{1}\right)$ and $W\left(\alpha_{2}\right)$ and $V$ an $s$-cobordism between $\partial W\left(\alpha_{1}\right)$ and $\partial W\left(\alpha_{2}\right)$,

(2) the lower triangle is also commutative such that $M \rightarrow V \rightarrow$ $\partial D^{i+2} \times I$ is a smooth fibration,

(3) $F \mid W\left(\alpha_{i}\right)(i=1,2)$ induces the object $\alpha_{i}(i=1,2)$.

We can introduce an operation $\alpha_{1} \# \alpha_{2}$ between two objects by taking a suitable connected sum along the boundaries. It is tedious but not difficult to see that the resulting cobordism group is nothing but $\pi_{i}(\mathscr{C}(M))$. If we require the map $f$ of the object $\alpha$ to be a simple homotopy equivalence and the map of the cobordism $F$ also to be a simple homotopy equivalence, we have a new cobordism group which is nothing but $\pi_{i}(\mathscr{B}(M))$. From here on, we shall always interpret $\pi_{i}(\mathscr{C}(M)), \pi_{i}(\mathscr{B}(M))$ to be the cobordism groups as just described. It is easy to derive a long exact sequence

$$
\begin{aligned}
& \longrightarrow L_{n+i+3}\left(\pi_{1} M, w\right) \longrightarrow \pi_{i}(\mathscr{B}(M)) \longrightarrow \pi_{i}(\mathscr{C}(M)) \\
& \longrightarrow L_{n+i+2}\left(\pi_{1} M, w\right)
\end{aligned}
$$

from these cobordism groups. (48) is just another description of (8).

THEOREM 4.1. Let $\alpha: W^{n+2} \rightarrow M^{n} \times\left(D^{2}, \partial D^{2}, 1\right)$ be an object representing an element of $\pi_{0}(\mathscr{C}(M))$ with $n+1=2 k$ or $2 k+1 \geqq 6$. Then, $\alpha$ is cobordant to an object (which is again denoted by $\alpha$ ) such that

(1) $p: W \rightarrow I^{2}$ has only the singularities of (B) of $\S$ III where $I^{2}$ is a product structure of $D^{2}$,

(2) the lines of handles are of index $k$ and $(k+1)$ only,

(3) the vertical tangents (with respect to the product structure $D^{2}=I^{2}$ ) corresponding to surgery birth-death points have lines of $k$-handles coming out of the top.

THEOREM 4.2. Let $F: U \rightarrow M \times\left(I^{2}, \partial I^{2}, 1\right) \times I$ be a cobordism of 
two objects satisfying the conclusion of Theorem 4.1 with $n+1=2 k$ or $2 k+1 \geqq 15$. Then, we may make $F$ satisfying the following conditions:

(1) $P: U \rightarrow I^{3}$ has only the singularities of (C) of $\S$ III,

(2) all the sheets of handles are of index $k$ and $(k+1)$ only,

(3) all the vertical tangents with respect to the product structure $\left(I^{2}, \partial I^{2}, 1\right) \times I$ have sheets of $k$ handles coming out of the top.

We need a lemma for the proofs of the above theorems.

LEMMA 4.3. If $f: W^{n+1} \rightarrow M \times I$ (with $n \geqq 6$ ) is a normal cobordism relative to the boundaries, then $f$ is cobordant to a map (also denoted by $f$ ) such that $p_{0} \cdot f: W \rightarrow I$ is a Morse function with singularities in the middle dimension only, where $p_{0}: M \times I \rightarrow I$ is the projection onto the second factor.

Proof. Perform surgery to make $f$ highly connected. It follows from Morse theory that $p_{0} \cdot f$ is homotopic to a Morse function with singularities of index $k$ and $k+1$ only for $n+1=2 k$ or $2 k+1$. Since $p_{0}$ is a fibration, this homotopy lifts to one of $f$. If $n+1=$ $2 k+1$, we are done. If $n+1=2 k$, it follows from the standard trick to eliminate the $(k+1)$-handles.

Proof of Theorem 4.1. Write $D^{2}$ as $I^{2}$ and perform surgery on $f: W^{n+2} \rightarrow M \times I^{2}$ to make it highly connected. By Lemma 4.3 , we can perform a homotopy of $z_{1} f$ to give a Morse function with only middle dimension handles. Using the fact that $p_{0}: M \times I^{2} \rightarrow I^{2}$ is a fibration, we can lift the homotopy to one of $f$ and assume that $z_{1} f$ is a Morse function with only middle dimensional handles. By a small homotopy of $f$ (not affecting our condition on $z_{1} f$ ), we may assume that $p: W \rightarrow I^{2}$ has only the singularities of (B) of $\S$ III. If $n+1=2 k$, using the fact that $z_{1} f$ is a Morse function with only middle dimensional handles, we get the following kinds of vertical tangents:

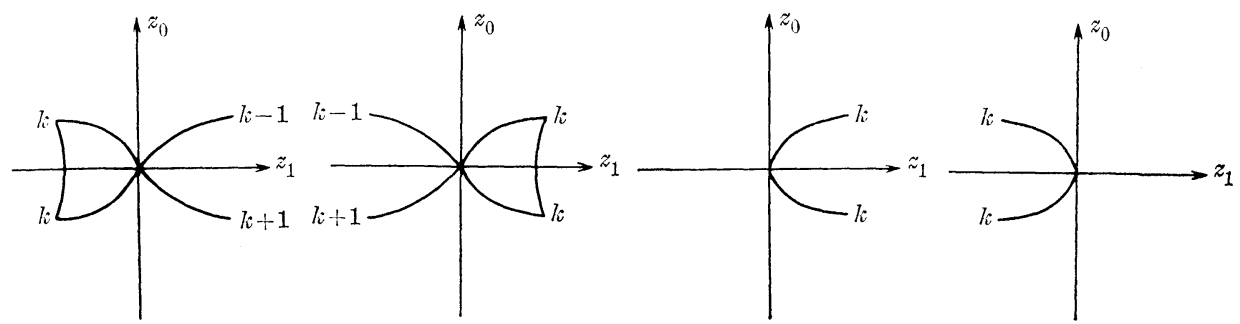

Figure 12

If $n+1=2 k+1$, we get the following kinds of vertical tangents: 

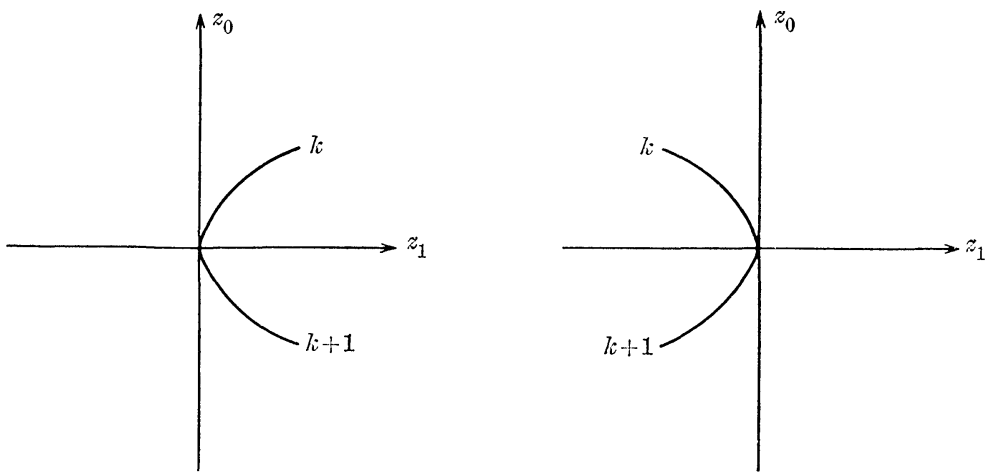

FIgURE 13

Thus, we obtain the condition regarding vertical tangents in the theorem. Following the argument of [3], we eliminate all lines of handles of $\operatorname{dim} \neq k, k+1$. This completes the proof of Theorem 4.1.

We need two more lemmas for the proof of Theorem 4.2.

Lemma 4.4. If $f: W \rightarrow M \times\left(I^{2}, \partial I^{2}\right)$ is as described in Theorem 4.1, then the number of cusps is even.

Proof. The number of the cusps together with the number of vertical tangents is even. We need only to show that the number of vertical tangents is even. If $(n+2)$ is even, the number of vertical tangents is equal to the rank of $K_{l}(W)(l=(n+2) / 2)$ where $K_{i}(\quad)$ denotes the kernel of $f_{*}$ [13]. So, we have proved the case $(n+2)$ being even. If $(n+2)$ is odd, then each slice is even dimensional and the rank of $K_{l}$ (slice) $(l=(n+1) / 2)$ changes by two as we pass a vertical tangent. Since this rank begins and ends with zero, there are even number of vertical tangents.

LEMmA 4.5. We may assume that there is no cusp in Theorem 4.1.

Proof. First assume $n+1=2 k+1$. We may introduce circles of singularities

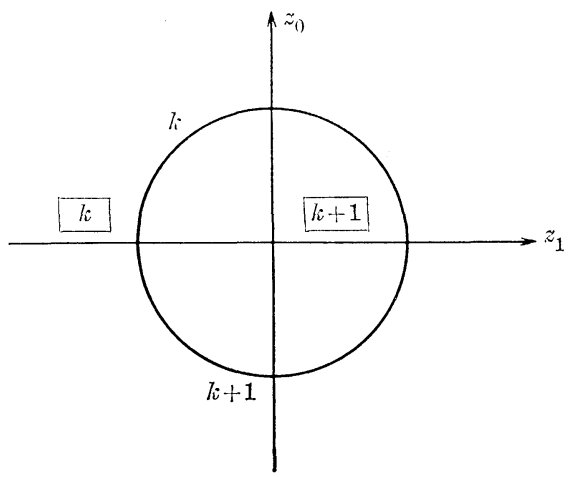

Figure 14 
at will, and then use swallow's tails to introduce a pair of cusps in the form

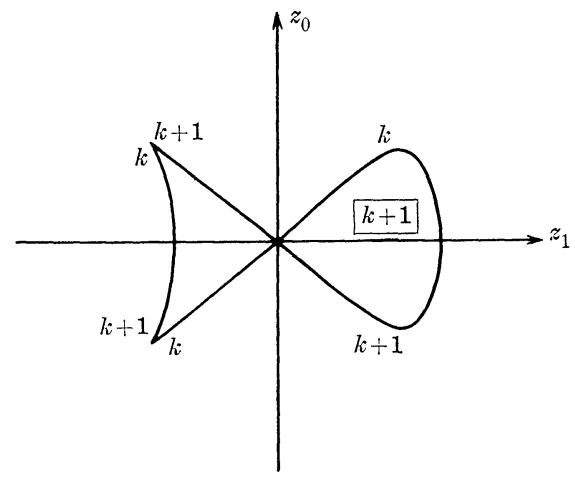

FIgURE 15

where $k, k+1$ in the box are the indices of the critical points of $z_{1} p$. Using these cusps, we can eliminate all the cusps by independence of birth' [2] [3]. None of these affects the other conditions of Theorem 4.1. Now assume $n+1=2 k$. In [3], Hatcher-Wagoner give a method for trading a line of $(k+1)$ handles for a line of $(k-1)$ handles by introducing a graphic of the form

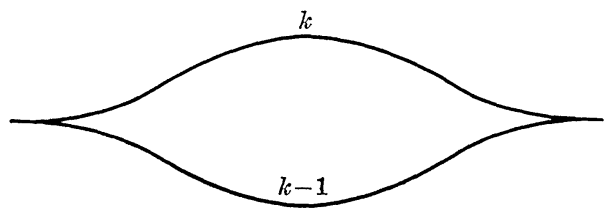

FIGURE 16

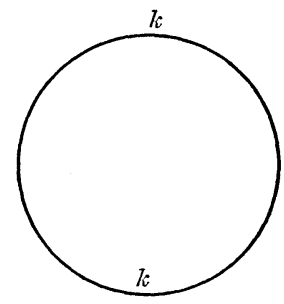

FIGURE 17

and then cancelling the $(k+1)$ handles with the newly created $k$ handles. We can do the same thing by introducing a circle of singularities we can eliminate all the $(k+1)$ handles without introducing $(k-1)$ handles. So, it eliminates all cusps. 
Proof of Theorem 4.2. Perform surgery on $F$ to make it highly connected. Make a homotopy of $F$ so that $\left(z_{1}, z_{2}\right) F$ satisfies the hypothesis of Lemma 4.5 and perform another small homotopy if necessary such that $P \cdot F$ has the singularities of (C) of $\S$ III and $\left(z_{1}, z_{2}\right) F$ has no cusp.

Now, assume $n+1=2 k$. The vertical tangents are of the form
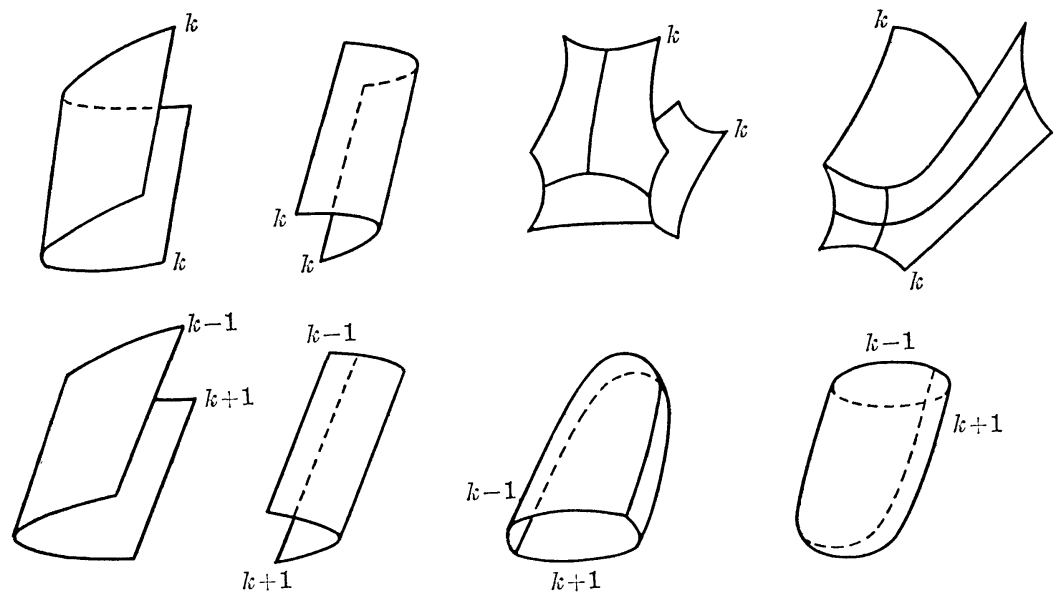

FIGURE 18

The vertical tangents for the last four form circles and we can change them to the first four by swallow's tails singularities. For example, we have the following change
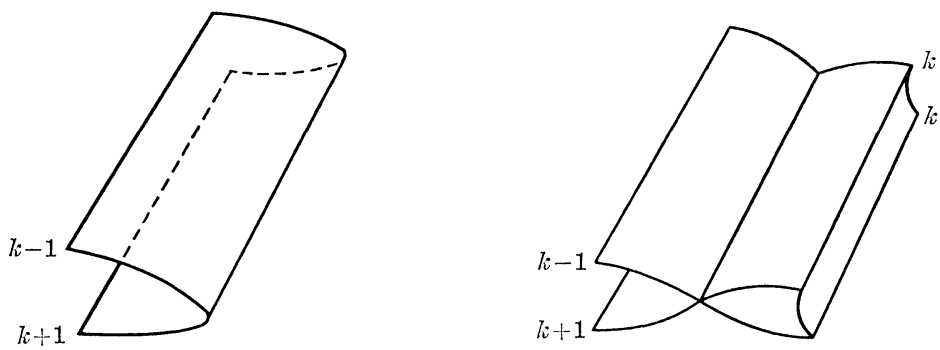

FIGURE 19

Thus, we have a sheet of index $k$ critical points coming out of the top for the vertical tangents.

Next, we consider the case $n+1=2 k+1$. The vertical tangents are of the form 

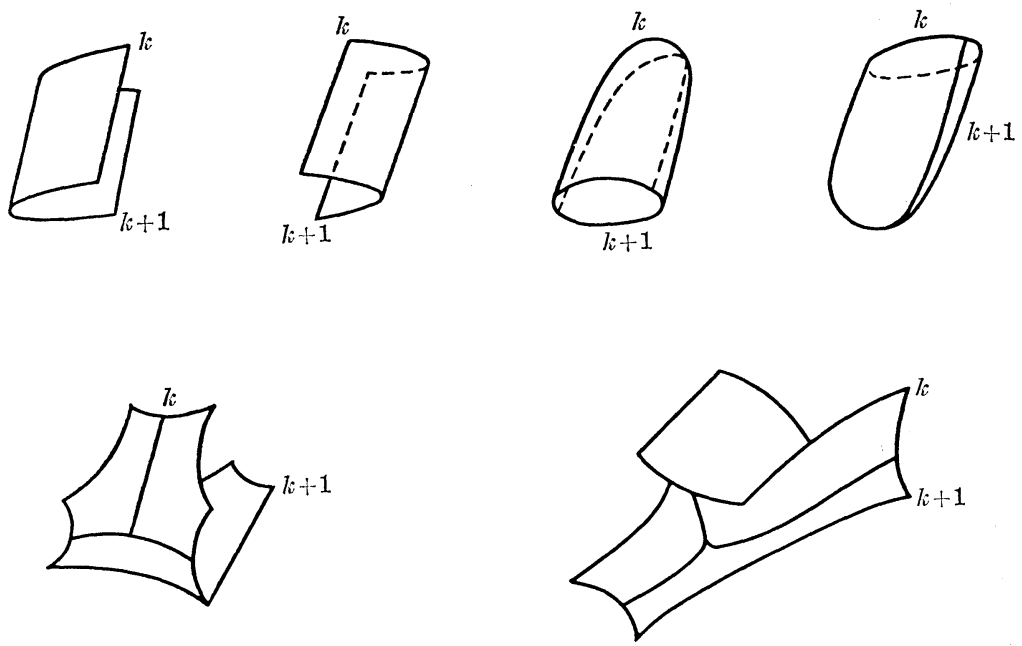

FIgURE 20.

So, again we have a sheet of index $k$ critical points coming out of the top for the vertical tangents. Applying the argument of [3], we can eliminate all sheets of the handles of index $\neq k, k+1$. This completes the proof of Theorem 4.2.

Let us now recall the cobordism representations of $\pi_{i}(\mathscr{B}(M))$. We consider an object $\beta=(W(\beta), f)$

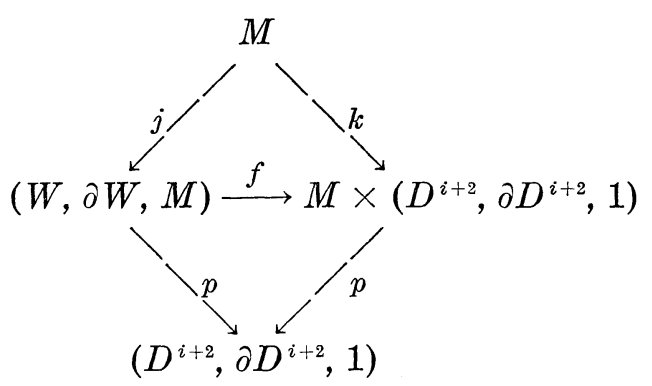

similar to that for $\pi_{i}(\mathscr{C}(M))$, but we require further that $f$ is a simple homotopy equivalence. Similarly, we require the cobordism between two objects

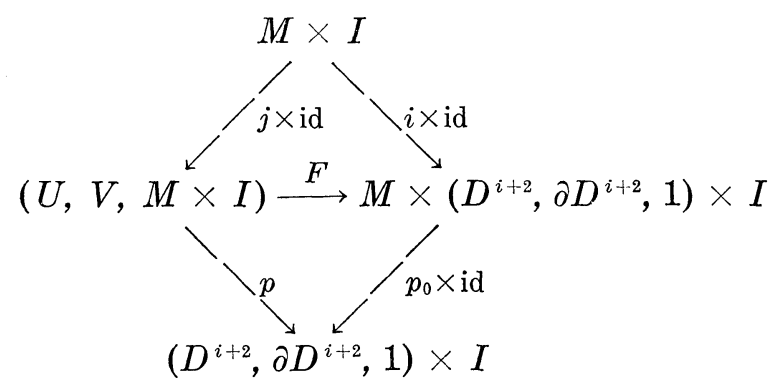


to satisfy the extra condition that $F$ is again a simple homotopy equivalence. It is easy to see that the cobordism group is nothing but $\pi_{i}(\mathscr{B}(M))$. Consider an object $\beta$ representing an element of $\pi_{0}(\mathscr{B}(M))$. Using the fact that $f$ is a simple homotopy equivalence and $f \cdot j=k$ is the standard inclusion, we can give a product structure $M \times I^{2}$ to $W$ such that $j: M \rightarrow \partial W$ is identified with $M \times(0,0)$ and $\beta$ is represented by a pseudo-isotopy problem of [3]. In fact, $p$ becomes the projection of the 1-parameter family of Morse function of [3] and the graphic has no vertical tangent. Using this particular pseudoisotopy representation, we have an element

$$
a \in W h_{2}\left(\pi_{1} M\right) \oplus W h_{1}\left(\pi_{1} M ; \Gamma\right)
$$

as the obstruction to isotopy to the identity. Following [3], if we give another pseudo-isotopy representation for the object $\beta$, we change $a$ to $a+g+(-1)^{n} \bar{g}$ for $g \in W h_{2}\left(\pi_{1} M\right) \oplus W h_{1}\left(\pi_{1} M ; \Gamma\right)$. In fact, if $\beta$ is changed to a cobordant object $\beta^{\prime}$, the obstructions run through the coset of $a$ in

$$
\left\{W h_{2}\left(\pi_{1} M\right) \oplus W h_{1}\left(\pi_{1} M ; \Gamma\right)\right\} /\left\{c+(-1)^{n} \bar{c}\right\} .
$$

So, we have the following theorem.

THEOREM 4.6. There is a natural isomorphism

$$
\dot{\rho}_{1}: \pi_{0}(\mathscr{B}(M)) \longrightarrow\left\{W h_{2}\left(\pi_{1} M\right) \oplus W h_{1}\left(\pi_{1} M ; \Gamma\right)\right\} /\left\{c+(-1)^{n} \bar{c}\right\} .
$$

V. Proof of Theorem 2.1 for $n$ even. Let us fix a cobordism representation $\alpha$

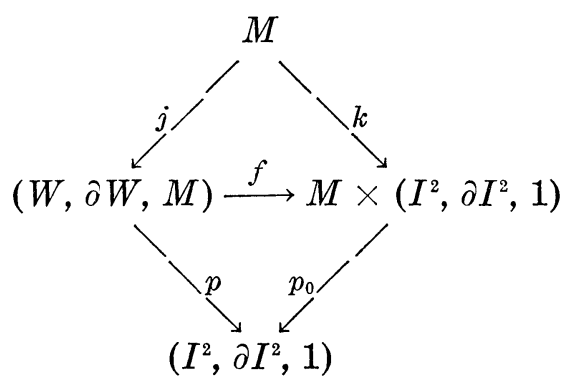

of an element $[\alpha] \in \pi_{0}(\mathscr{C}(M))$ where $I^{2}$ is a specific identification of $I^{2}$ with $D^{2}$. Assume that $\alpha$ satisfies the conclusion of Theorem 4.1. We set

$$
W_{t}=p^{-1}(\{t\} \times I)=f^{-1}(M \times\{t\} \times I)
$$

and call it the $t$-slice of $W$. If $\{t\} \times I$ contains no cusp or vertical 
tangent, then it is an $(n+1)$-dim manifold and the induced map

$$
f_{t}: W_{t} \longrightarrow M \times\{t\} \times I
$$

is a highly connected normal map. There exist $0<t_{1}<t_{2}<\cdots<$ $t_{r}<1$ such that cusps, vertical tangents, handle additions and regular homotopies only occur at these moments and once for each time. A $t$-slice for $t \neq t_{1}, \cdots, t_{r}$ is called a regular slice. Choose a gradient-like vector field $\xi$ (§III) on $W$ such that for $t$ slightly away from $t_{i}(i=1, \cdots, r)$ the descending manifolds of the critical points are in general position in

$$
\partial_{-} W_{t}=p^{-1}(\{t\} \times 0)=f^{-1}(M \times\{t\} \times 0) .
$$

Let us examine how the descending manifolds in $\partial_{-} W_{t}$ look. At a $t$-slice slightly to the right of a surgery birth point, the descending manifold of index $k$ from this surgery birth point is a framed $(k-1)$-sphere embedded in $\partial_{-} W_{t}$. We call it the $k$-bottom of this surgery birth. The descending manifold of index $(k+1)$ slightly to the right of a surgery birth comes down via the descending trajectories and is punctured where it meets the ascending trajectories of critical points of index $k$. So we have an embedded framed punctured $k$-sphere in $\partial_{-} W_{t}$. The $k$-caps of the critical points which puncture our $k$-sphere plug the holes such that it represents an immersed framed $k$-sphere in $\partial_{-} W_{t}$. Again, it bounds an immersed framed $(k+1)$-disc, unique up to regular homotopy. We fix one and call it the $(k+1)$-bottom of the surgery birth.

Similarly, we can define the $k$-bottom, $k$-cap, $(k+1)$-bottom, and $(k+1)$-cap of a usual birth point, i.e., cusp pointing to the left. The $k$-cap can simply be gotten from the $(k+1)$-bottom by pushing it out slightly, and the $(k+1)$-cap is just the trace of pushing out. From the independence of birth [3] we may assume the $k$-cap and $(k+1)$-cap are honestly embedded $k$-disc and $(k+1)$ disc, respectively.

When $t$ moves to the right the various $k$-caps and $(k+1)$-caps move toward their death points in a definite fashion which we shall describe shortly. We shall record the geometric movements of $k$ caps and $(k+1)$-caps algebraically, and eventually we shall have a complete invariant for $[\alpha] \in \pi_{0}(\mathscr{C}(M))$.

Let us fix an orientation for $W$ at the base point. Order all the critical points of index $k$ right after their birth, and order the correspoding critical points of index $(k+1)$ accordingly. Choose paths from the critical points to the base point compatibly over their life time: Fix a path from a critical point of index $k$ right after its birth and fix the path from the corresponding critical point 
of index $(k+1)$ by going around the (surgery or usual) birth point. Then, the path from a critical point in a $t$-slice is gotten by following the critical line back to the initial critical point followed by the path from this critical point. Fix the orientations of the descending manifolds compatibly by means of the paths. We also orient the tangent space of each critical point in a slice such that the rightward normal to the slice $\oplus$ the orientation of the tangent space of the slice is the orientation at the base point transported along the above path. We also orient the ascending manifold of the critical point such that its orientation $\oplus$ the orientation of the descending manifold is the orientation of the tangent space of the critical point. Finally, we observe that each critical submanifold meets each level surface generically in a $(k-1)$ or $k$ dimensional submanifold, which we orient according to: upward normal $\oplus$ the orientation of the intersection is the orientation of the critical submanifold.

Now, we embark on a program to describe how the critical submanifolds fit together in a $t$-slice $W_{t}$ by $k$-caps, $(k+1)$-caps and their changes. We consider in turn the case of a regular slice and seven varieties of singular slices.

(A) A regular slice. Let $W_{t}$ be a regular slice. Inductively, we assume that $k$-caps and $(k+1)$-caps are given for this slice. Let us describe how the critical submanifolds fit together in terms of them. The phenomena persist until we meet the next slice $W_{t_{i}}(i=1, \cdots, r)$. In the next few paragraphs, we shall describe the changes when we pass these singular slices. The critical submanifolds fit together in two fundamental ways on a regular slice. We refer to these as a $(k+1)$-handle being attached along a $k$-handle or across a $k$-handle.

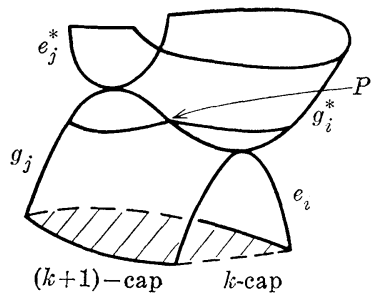

$k$-cap plugs the $(k+1)$ bottom of a $(k+1)$-cap - a $(k+1)$-handle being attached a $k$-handle.

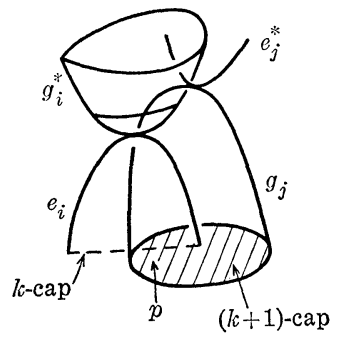

$k$-cap cuts across the $(k+1)$-bottom of a $(k+1)$ cap — a $(k+1)$-handle being attached across a $k$-handle.

FIGURE 21 
The critical submanifolds fit together transversely along a common part $P$. In the first case, $P$ is a union of an interval and two $k$ discs. We shall mostly be concerned with this intersection in a level surface between the critical levels involved, where $P$ now is equal to a single point intersection between two framed $k$-spheres in a $2 k$-dim manifold. In the second case, $P$ is equal to a single point intersection between a framed $k$-disc and a (possibly punctured) $k$-sphere in a $2 k$-dim manifold. Call such intersections persistent since they persist to nearby slices. Each handle may be involved in both kinds of intersection many times and we count each time separately. Let $e_{i}$ be the framed descending manifold of the $i$ th critical point of index $k$ together with the corresponding framed immersed $k$-disc $D_{i}^{k}=$ the $i$ th $k$-cap in $\partial_{-} W_{t}$. Moving along the ascending trajectories slightly, we may separate the intersections of the $k$-caps and we have an embedded framed $k$-sphere for $e_{i}$. Let $g_{i}$ be the framed descending manifold of the $i$ th critical point of index $(k+1)$ together with the corresponding framed $(k+1)$-disc $D_{i}^{k+1}=$ the $i$ th $(k+1)$-cap in $\partial_{-} W_{t} . \quad\left(e_{j}^{*}, g_{j}^{*}\right.$ in Figure 21 denote the ascending manifolds.)

Let $U$ be the boundary connected sum of the normal disc bundles of the $e_{i}$ 's, connected along the paths to the base point. The framings and the orientations determine classes $\left[e_{i}\right],\left[f_{i}\right] \in K_{k}(\partial U)$ with $\left[e_{i}\right]\left[f_{j}\right]=\delta_{i j}$ and $\left[g_{j}\right] \in K_{k+1}\left(W_{t}, U\right)$ [13]. Following [12], we obtain an element $A \in \widetilde{S U}\left(\pi_{1} W\right)$, where

$$
A=\left(\left[\begin{array}{ll}
\alpha * \\
\gamma *
\end{array}\right]\left[\begin{array}{l}
{[\alpha]} \\
{[*]}
\end{array}\right)\right.
$$

represents the Wall surgery invariant for the normal map obtained by the restriction to this slice; we shall call it the slice invariant. Note that for $e_{i}, g_{i}$ not in this slice, we fill out $\gamma$ by 0 at the $i$ th column and $\alpha$ by $\delta_{i j}$ at the $i$ th column. (Of course, $A$ represents the 0 obstruction since the surgery problem is visibly trivial.)

The slice invariant can be obtained by measuring the intersections as follows. If $P$ is a persistent intersection, let $e_{P}= \pm 1$ be the sign of the intersection at $P$ in a level surface, and let $g_{P} \in \pi_{1} M$ be the class of the path from $e_{i}$ to $g_{j}$ via $P$.

$$
\begin{array}{ll}
\alpha_{i j}=\sum_{P} \varepsilon_{P} g_{P} & \text { summing over } P \text { where } \\
\gamma_{i j}=\sum_{P} \varepsilon_{P} g_{P} \text { attached along } c_{i}, & \text { summing over } P \text { where } \\
& g_{j} \text { attached across } e_{i} .
\end{array}
$$

Moreover, there are certain intersections between $D_{j}^{k+1}$ (the $j$ th 
$(k+1)$-cap) and $\partial D_{i}^{k+1}$ (the $i$ th $(k+1)$-bottom) consisting of circles and intervals which will be counted. We only use the nontrivially covered circles and the intervals joining $e_{p} \subset \partial D_{j}^{k+1}$ to $e_{q} \subset \partial D_{i}^{k+1}$ (for any $p, q$ ). Such a component $Q$ determines $\varepsilon_{Q}= \pm 1$ by comparing the orientations of

$$
\tau\left(\partial D_{i}^{k+1}\right) \oplus\left(\tau\left(D_{j}^{k+1}\right) / \tau(Q)\right)
$$

and $\tau\left(\partial_{-} W_{t}\right)$ at $Q$ using the path from $g_{i}$ to the base point. Also $g_{Q} \in \pi_{1} W$ is determined by the path from $\partial D_{i}^{k+1}$ to $D_{j}^{k+1}$ via $Q$. Then,

$$
a_{i j}=\sum_{Q} \varepsilon_{Q} g_{Q} \quad \text { summing over } Q \text { of the above. }
$$

For simplicity, we supress the reference to the form $[a]=\left[a_{i j}\right]$ for the discussion. See [12] for more details. If $t \in\left(t_{q}, t_{q+1}\right)$, then the slice invariant is independent of $t$, and we denote it by $A_{q}$. Altering the order, orientation, and base point path of the critical points we replace $e_{j}$ by $e_{i}\left(\varepsilon_{j i}\left(s_{(j i)}\right)\right)$ for $\varepsilon_{j i}= \pm 1, \quad s_{(j i)} \in \pi_{1} W$ or $g_{j}$ by $g_{i}\left(\varepsilon_{j i}\left(s_{(j i)}\right)\right)$. Thus, we replace $A$ by $H(\tau)^{*} A$ or $A H(\tau)$ where $\tau$ is a generalized permutation matrix $\tau=\left(\varepsilon_{i j} s_{(i j)}\right)$ and $H$ is the homomorphism $^{5}$ defined in [13].

In addition to the slice invariant $A_{q}$ which is independent of $t \in\left(t_{q}, t_{q+1}\right)$, there is another invariant, the slab invariant $Z^{q}$ which is gotten by looking at the whole slab $\left[t_{q}, t_{q+1}\right]$ at once. $Z^{q}$ will be written as

$$
\left[\begin{array}{l}
X^{q} \\
Y^{q}
\end{array}\right]=\left[\begin{array}{l}
\left(x_{i j}^{q}\right) \\
\left(y_{i j}^{q}\right)
\end{array}\right]
$$

with the entries $x_{i j}^{q}, y_{i j}^{q} \in\left(Z_{2} \times \pi_{2} M\right)\left[\pi_{1} W\right]$ of [4]. Its definition depends heavily on certain choices to be made in the singular slices $t_{q}$ and $t_{q+1}$. At each singular slice, there are various persistent intersections $P$ which come from the left and go to the right. Each persistent intersection is equipped with a loop (from $g_{i}^{*}$ to $g_{j}$ or from $e_{\imath}$ to $g_{j}$ via $P$ ). At a level surface, each component of the persistent intersection over the slab $\left[t_{q}, t_{q_{+1}}\right]$ has two framings either gotten from the normal bundle $\nu\left(g_{j}\right)$ of $g_{j}$ and the tangent bundle $\tau\left(g_{i}^{*}\right)$ of $g_{i}^{*}$, or gotten from the normal bundle $\nu\left(g_{j}\right)$ of $g_{j}$ and the tangent bundle $\tau\left(e_{i}\right)$ of $e_{i}$. At a singular slice, we choose: (1) deformations of the loops to canonical loops, (2) deformations of the framings to equal framings (up to the sign of the last vector). Following [4], we can associate to each component of a persistent intersection $P$ over $\left[t_{q}, t_{q+1}\right]$ an element

$$
\left(u_{P}, \varepsilon_{P} v_{P}\right) g_{P} \in\left(\boldsymbol{Z}_{2} \times \pi_{2} W\right)\left[\pi_{1} W\right]
$$

${ }^{5} h(\quad)$ is the lifting of $H(\quad)$ to the Steinberg group. Some times we identify them if no confusion aries. 
where $\varepsilon_{P}, g_{P}$ are as before, $u_{P} \in Z_{2}$ comes from the comparison of the framings $\left(Z_{2}=\pi_{1}(G L(R))\right)$, and $v_{p} \in \pi_{2}(W)$ comes from the comparison of the two ways of deforming the loop $P$ to the canonical loop (one via $g_{i}^{*}$ and the other one via $g_{j}$, or one via $e_{i}$ and the other one via $g_{j}$ ). Then,

$$
\begin{cases}x_{i j}^{q}=\sum_{P}\left(u_{P}, \varepsilon_{P} v_{P}\right) g_{P} & \text { summing over } P \text { where } g_{j} \\ y_{i j}^{q}=\sum_{P}\left(u_{P}, \varepsilon_{P} v_{P}\right) q_{P} & \text { is attached along } e_{i}, \\ & \text { summing over } P \text { where } g_{j} \\ \text { cuts across } e_{i} .\end{cases}
$$

Of course, the slab invariant depends on the choices made at the singular slices. We shall discuss the change in the next few paragraphs where we describe the nature of each singular slice.

(B) Geometric cancellations I.

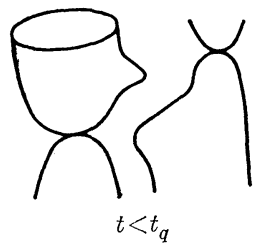

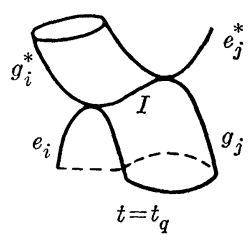

FIGURE 22

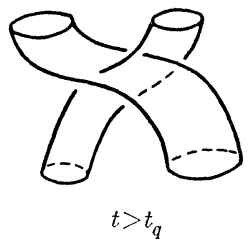

On the singular slice $t_{q}$ of the above deformation, there is an instantaneous intersection $I$ between $g_{j}$ and $g_{i}^{*}$ (gotten by the deformation of the gradientlike vector field $\xi$ ) between the ascending and descending $k$-spheres in a level surface between the critical points. This results in the appearance of two persistent intersections $P_{ \pm}$ for $t>t_{q}$ where $g_{j}$ is attached along $e_{i}$. Let $g_{I} \in \pi_{1} W$ be the path from $e_{i}$ to $g_{j}$ via $I$. Then, $P_{ \pm}$receive invariants

$$
\left\{\begin{array}{l}
g_{P_{ \pm}}=g_{I} \\
\varepsilon_{P_{ \pm}}= \pm 1
\end{array}\right.
$$

so that $\varepsilon_{P_{+}} g_{P_{+}}+\varepsilon_{P_{-}} g_{P_{-}}=0$. Thus, the slice invariant is unchanged by this kind of singularity. Both $P_{ \pm}$begin life as $I$, so the choice of the deformation of the loops and the framings of $P_{ \pm}$are equal. The only slab invariant which can be altered by varying the choices is $x_{i j}^{q}$ in $Z^{q}$. In fact, varying our choices by $(u, v) \in \boldsymbol{Z}_{2} \times \pi_{2} W$ changes $x_{i j}^{q}$ by

$$
\left(u, \varepsilon_{P_{+}} v\right) g_{P_{+}}+\left(u, \varepsilon_{P_{-}} v\right) g_{P_{-}}=(2 u, v-v) g_{I}=0 .
$$

Hence, $Z^{q-1}+Z^{q}$ is independent of the choices made at $t_{q}$. 
(C) Geometric cancellation II.

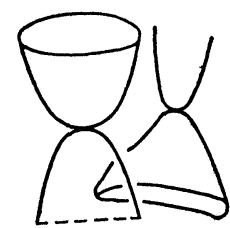

$t<t_{q}$

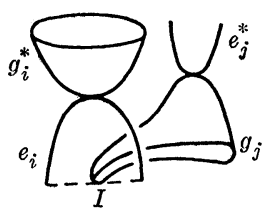

$t=t_{q}$

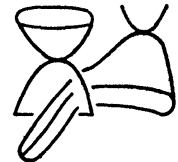

$t>t_{q}$

FIGURE 23

On the singular slice $t_{q}$ of the above deformation, there is an instantaneous intersection $I$ between $e_{i}$ and $g_{j}$ corresponding to a regular homotopy of the $i$ th $k$-cap $D_{i}^{k}$ and the $j$ th $(k+1)$-bottom in $\partial_{-} W_{t}$. This results in the appearance of two persistent intersections $P_{+}, P_{-}$. Let $g_{I} \in \pi_{1} W$ be the path from $e_{i}$ to $g_{j}$ via $I$. Then, $P_{ \pm}$receive invariants

$$
\left\{\begin{array}{l}
g_{P_{ \pm}}=g_{I} \\
\varepsilon_{P_{ \pm}}= \pm 1
\end{array}\right.
$$

so that the slice invariant does not change. Again, both $P_{ \pm}$begin life as $I$ so we choose the deformation of the loop and the framings to be equal. Exactly as before, the sum $Z^{q-1}+Z^{q}$ is independent of any choice made at $t_{q}$.

(D) Handle addition I $(k / k)$.

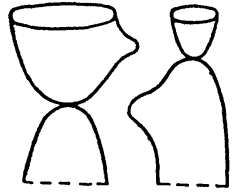

$t<t_{q}$

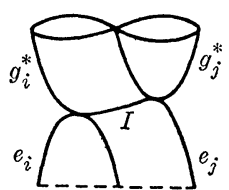

$t=t_{q}$

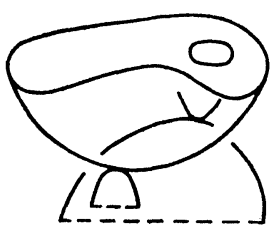

$t>t_{q}$

FIGURE 24

On the singular slice $t_{q}$, there is an instantaneous intersection $I$ between $g_{i}^{*}$ and $e_{j}$ (in fact, $g_{i}^{*} \supset g_{j}^{*}$ and $e_{i} \supset e_{j}$ ). If $P$ is a persistent intersection, corresponding to a $(k+1)$-handle $g_{s}$ attached along $e_{j}$ (respectively across $e_{i}$ ), then it splits into two after passing the singular slice, with one copy for the original intersection, and the other for the new one where $g_{s}$ is attached along $e_{i}$ (respectively across $e_{j}$ ). Let $g_{I} \in \pi_{1} W$ come from the path from $e_{j}$ to $g_{i}^{*}$ via $I$, $\varepsilon_{I}= \pm 1$ compare the orientations of $g_{i}^{*}$ and $g_{j}^{*} \subset g_{i}^{*}$. Thus, we have new terms describing how $g_{s}$ is attached along $e_{i}$ and across $e_{j}$, which are $\varepsilon_{I} g_{I} \alpha_{j s}$ and $-\varepsilon_{I} \bar{g}_{I} \gamma_{i s}$. Thus, the effect on the slice invariant is multiplying from the left by $H(E)$ where 


$$
E=I+E_{i j}\left(\varepsilon_{I} g_{I}\right)
$$

is the elementary matrix with $\varepsilon_{I} g_{I}$ in the $(i, j)$ th spot.

Following [4], we make the loops at the new intersections homotopic to the product of the loop at $I$ with the loops at the old intersections, and we can give framings of the new intersections such that the slab invariants $Z^{q-1}$ and $Z^{q}$ over $\left[t_{q-1}, t_{q}\right]$ and $\left[t_{q}, t_{q_{+1}}\right]$ are defined. Let us now consider the matrix

$$
A_{q-1}^{-1} Z^{q-1}+A_{q}^{-1} Z^{q}
$$

where $A_{q-1}, A_{q}$ are the slice invariants of $\left(t_{q_{-1}}, t_{q}\right),\left(t_{q}, t_{q+1}\right)$ respectively. (69) is a matrix with entries in $\left(Z_{2} \times \pi_{2} W\right)\left[\pi_{1} W\right]$ and its trace

$$
\operatorname{Tr}\left(A_{q-1}^{-1} Z^{q-1}+A_{q}^{-1} Z^{q}\right)
$$

is defined and can be viewed as an element of $W h_{1}\left(\pi_{1} W ; \Gamma\right)$ [4]. Let us check what is the effect on (70) if we renormalize at $I$, it follows from [4] that we may assume that $Z_{q-1}$ is unchanged but $Z^{q}$ is replaced by

$$
Z^{q}+H\left(E_{i j}(u, v) \varepsilon_{I} g_{I}\right)\left(\begin{array}{l}
\alpha \\
\gamma
\end{array}\right)
$$

where $A_{q}=\left(\begin{array}{ll}\alpha & \beta \\ \gamma & \delta\end{array}\right),(u, v) \in \boldsymbol{Z}_{2} \times \pi_{2} W$. Thus (70) is changed to

$$
\operatorname{Tr}\left(A_{q-1}^{-1} Z^{q-1}+\left(A_{q}^{-1} Z^{q}+A_{q}^{-1} H\left(E_{i j}(u, v) \varepsilon_{I} g_{I}\right)\left(\begin{array}{c}
\alpha \\
\gamma
\end{array}\right)\right) .\right.
$$

It is not difficult to check that (70) is equal to $(72)$ in $W h_{1}\left(\pi_{1} W ; \Gamma\right)$.

(E) Handle addition II $((k+1) /(k+1))$.

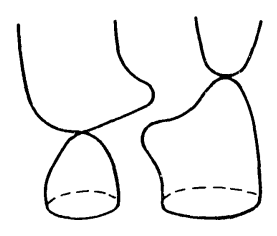

$t<t_{q}$

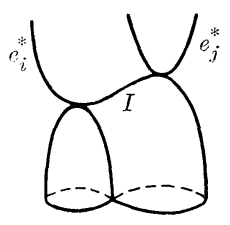

$t=t_{q}$

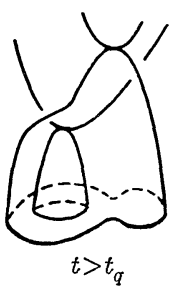

FIGURE 25

On the singular slice, there is an instantaneous intersection $I$ between $e_{i}^{*}$ and $g_{j}$. Let $g_{I} \in \pi_{1} W$ come from the path from $e_{i}^{*}$ to $g_{j}$ via $I$ and $\varepsilon_{I}= \pm 1$ compare the orientations of $e_{i}^{*}$ and $e_{j}^{*} \subset e_{i}^{*}$. The effect on the slice invariant is multiplying from the right by 
$H\left(I+E_{i j}\left(\varepsilon_{I} g_{I}\right)\right)$. Similar to the previous case, we may assume that the change of $A_{q-1} Z^{q-1}+A_{q}^{-1} Z^{q}$ by renormalization at $I$ is in $A_{q}^{-1} Z^{q}$ only. It replaces $Z^{q}$ by

$$
Z^{q}+\left(\begin{array}{l}
\alpha(u, v) E_{0} \\
\gamma(u, v) E_{0}
\end{array}\right)
$$

where $E_{0}=E_{i j}\left(\varepsilon_{I} g_{I}\right)$ and $(u, v) \in Z_{2} \times \pi_{2} W$. It is easy to check that $\operatorname{Tr}\left(A_{q-1}^{-1} Z^{q-1}+A_{q}^{-1} Z^{q}\right) \in W h_{1}\left(\pi_{1} W ; \Gamma\right)$ is again invariant.

(F) Regular homotopy. In $\partial_{-} W_{t}$, we have the following situation during a regular homotopy:
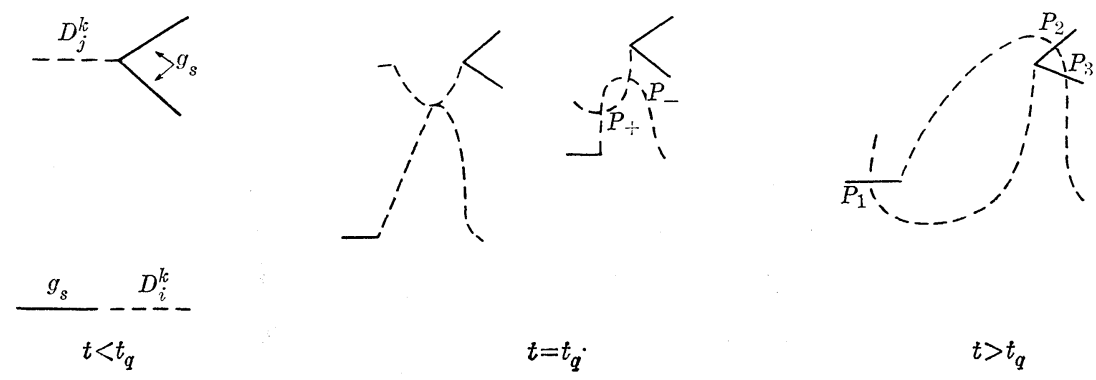

$$
t=t_{q}
$$$$
t>t_{q}
$$

FIGURE 26

On the singular slice $t_{q}$, there is an instantaneous intersection $I$, which evolves two new intersection $P_{ \pm}$. Move them across the edges of $D^{k}$ 's to the $g_{s}$ 's. This yields a collection of new intersections $P_{1}, \cdots$ corresponding to " $g_{s}$ is attached across $e_{i}$ or $e_{j}$ ". Let $g_{I} \in \pi_{1} W$ come from the path from $e_{i}$ to $e_{j}$ via $I \varepsilon_{I}= \pm 1$ according to $P_{+}$stays on $e_{i}$ or $e_{j}$. The invariants for $P_{ \pm}$are $g_{P_{ \pm}}=g_{I}$ and $\varepsilon_{P_{ \pm}}= \pm \varepsilon_{I}$. We end up with new terms describing how $g_{s}$ is attached across $e_{i}$ and $e_{j}$ which are $\varepsilon_{I} g_{I} \alpha_{s j}$ and $(-1)^{k+1} \varepsilon_{I} \bar{g}_{I} \alpha_{s i}$ respectively, and the effect on the slice invariant is multiplying from the left by

$$
\left(\left[\begin{array}{cc}
I & 0 \\
D+(-1)^{k+1} D^{*} & I
\end{array}\right]\left[\begin{array}{c}
{[D]} \\
{[*]}
\end{array}\right)\right.
$$

where $D$ is the matrix with $\varepsilon_{I} g_{I}$ in the $(i, j)$ th spot, and zero elsewhere.

The loop at $P_{1}$ is homotopic to the composite of the loop at $P_{-}$ and the loop between $D_{i}^{k}$ and $g_{s}$. We have an obvious framing at $P_{1}$ gotten from $P_{-}$(and hence from $I$ ). Similarly, we have the loops and framings for the points $P_{2}, \cdots$. Renormalizing at $I$, we only change the component $Y^{q}$ of $Z^{q}$ to $Y^{q}+((u, v) D+$ $(-1)^{k+1}[(u, v) D]^{*} \quad$ where $(u, v) \in \boldsymbol{Z}_{2} \times \pi_{2} W$. One can check that $\operatorname{Tr}\left(A_{q-1}^{-1} Z^{q-1}+A_{q}^{-1} Z^{q}\right)$ in $W h_{1}\left(\pi_{1}(W) ; \Gamma\right) /\{c+\bar{c}\}$ remains invariant. 
(G) Birth-death. The following pictures are sketches for a usual birth point. (Death point is similar.)
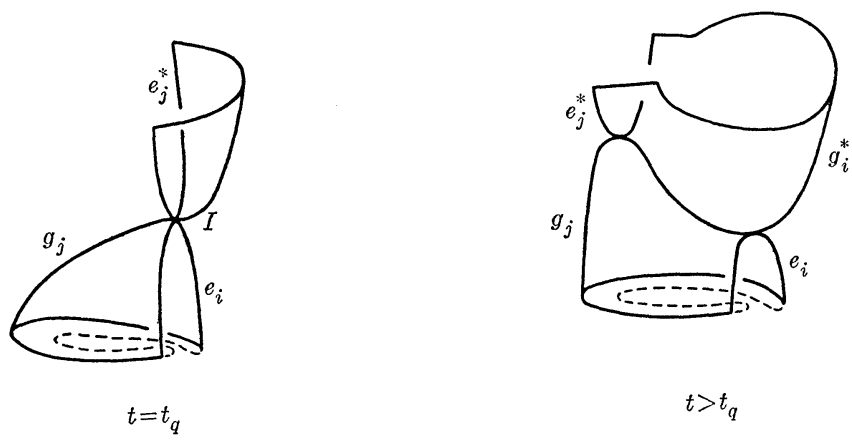

$t>t_{q}$

FIGURE 27

On the singular slice, there is an instantaneous intersection $I$ between $e_{i}$ and $e_{j}^{*}$. Moments later, we have a new intersection with $g_{j}$ being attached along $e_{i}$. It follows from [3] that the effect on the slice invariant is multiplying from the left by $H(E)$ where $E$ is a generalized permutation matrix. Moreover, $\operatorname{Tr}\left(A_{q-1}^{-1} Z^{q-1}+A_{q}^{-1} Z^{q}\right)$ is unchanged under renormalization of $I$. Death point is similar.

(H) Surgery on a k-sphere. The following pictures are sketches of a surgery birth. (Surgery death is similar.)

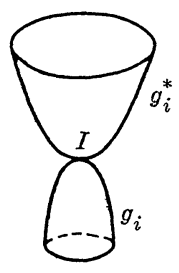

$t=t_{q}$
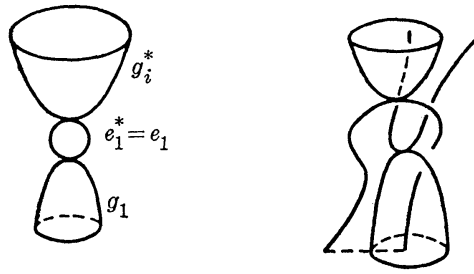

$t>t_{q}$

Figure 28

For convenience of notation, we assume that indices involved are all 1 so that the invariant before surgery is

$$
\left[\begin{array}{ll|ll}
1 & 0 & 0 & 0 \\
0 & \alpha & 0 & \beta \\
\hline 0 & 0 & 1 & 0 \\
0 & \gamma & 0 & \delta
\end{array}\right]
$$

One can easily reduce the general case to this case. On the singular slice, there is an instantaneous intersection $I$ between $g_{1}^{*}$ and $g_{1}$. 
Let $g_{I} \in \pi_{1} W$ come from the path from $g_{1}^{*}$ to $g_{1}$ via $I$, and let $\varepsilon_{I}= \pm 1$ come from the comparison of the orientations of $e_{1}$ and $e_{1}^{*}$. In $\partial_{-} W_{t}$, we have the following corresponding picture of Figure 28:
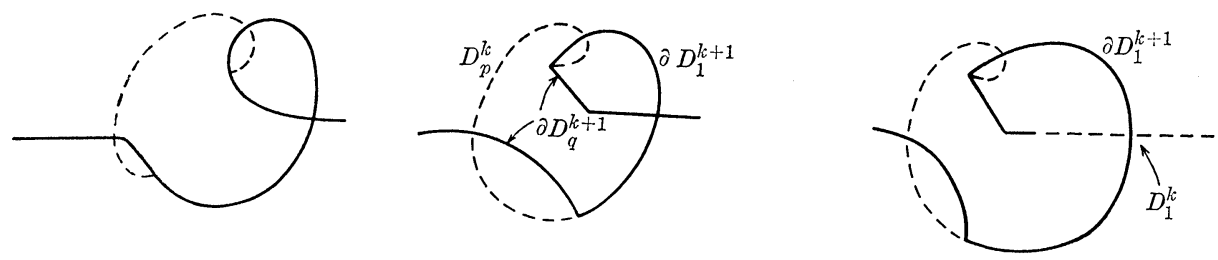

FIgURE 29

Let $b_{q}$ (respectively $c_{q}$ ) measure how $g_{1}$ is attached along (respectively across) $e_{q}(q>1)$ and let $b$ and $c$ be the corresponding column vectors. Then $g_{q}(q>1)$ is attached along (respectively across) with intersection number $-\varepsilon_{I} g_{I}\left(b^{*} \gamma+(-1)^{k} c^{*} \alpha\right)$ (respectively 0 ). Here we are using the fact that the algebraic intersection number of $\partial D_{q}^{k+1}$ and $\partial D_{1}^{k+1}$ is zero. Then, the effect on the slice invariant is to left multiply by

(76)

$$
\sigma_{11}(b, c)=\left[\begin{array}{cc|cc}
0 & 0 & \varepsilon_{I} g & 0 \\
0 & 1 & 0 & 0 \\
\hline(-1)^{k} \varepsilon_{I} \bar{g}_{I} & 0 & 0 & 0 \\
0 & 0 & 0 & 1
\end{array}\right]\left(\left[\begin{array}{cc|cc}
1 & 0 \\
b & 1 & 0 & 0 \\
0 & 0 \\
\hdashline 0(-1)^{k+1} c^{*} & \begin{array}{cc}
-b^{*} \\
c \\
c
\end{array}
\end{array}\right]\left[\begin{array}{ll}
\xi & 0 \\
c & 0
\end{array}\right]\right)
$$

where $\xi$ is the intersection number between $D_{1}^{k+1}$ and $\partial D_{1}^{k+1}$. Changes for new intersection points replace $Z^{q}$ by its sum with a matrix of the form

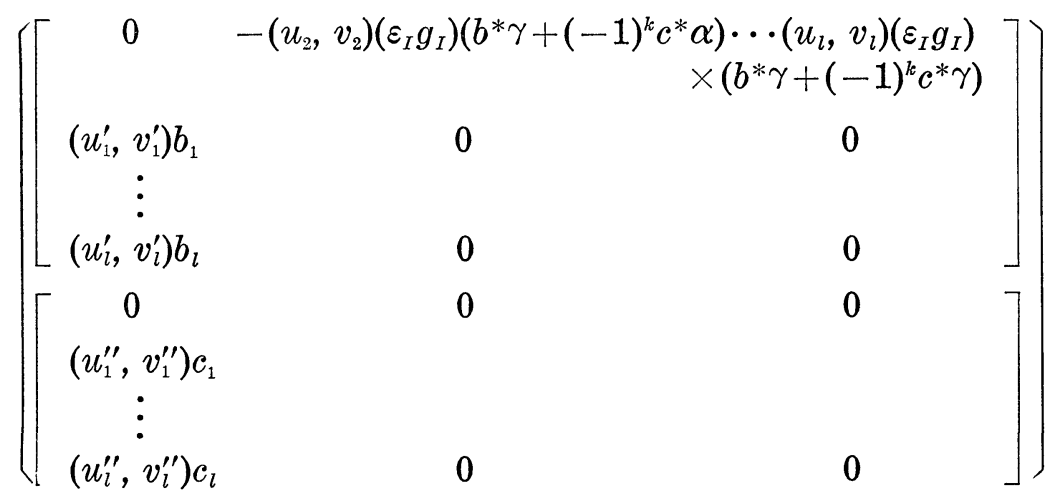

where $\left(u_{i}, v_{i}\right),\left(u_{i}^{\prime}, v_{i}^{\prime}\right),\left(u_{i}^{\prime \prime}, v_{i}^{\prime \prime}\right) \in Z_{2} \times \pi_{2} W$. It is clear that $\operatorname{Tr}\left(A_{q-1}^{-1} Z^{q-1}+\right.$ $\left.A_{q}^{-1} Z^{q}\right)$ is invariant under renormalization of $I$. 
(I) The invariants $\left.\theta \in L_{n}^{s t}\left(\pi_{1} W\right), W\right)$ and $\zeta \in W h\left(\pi_{1}(W) ; \Gamma\right)$. In the preceding paragraphs, we have defined the slice invariant $A_{q_{-1}}$ (in $\left(t_{q-1}, t_{q}\right)$ ) which changes to $A_{q}$ as we pass the singular slice $t_{q}$. In each case, $A_{q}=E_{q} A_{q-1} F_{q}$ where $E_{q}, F_{q}$ are 'elementary unitary matrices' which can be lifted to $\hat{E}_{q}, \hat{F}_{q} \in \widetilde{S t U}\left(\pi_{1} W\right)$. Let $A_{0}=i d$. $A_{r}$ is a generalized permutation matrix which is lifted to $\hat{A}_{r} \in$ $\widetilde{S t U}\left(\pi_{1} W\right)$. But

$$
\hat{\hat{A}}_{r}=\hat{E}_{r} \cdots \hat{E}_{1} \hat{A}_{0} \hat{F}_{1} \cdots \hat{F}_{r}
$$

is another lifting of $A_{r}$. So, we have a unique element $\theta \in$ $L_{n}^{s t}\left(\pi_{1}(W) ; W\right)$ by comparing $\hat{A}_{r}$ and $\hat{\hat{A}}_{r}$. Note that changing the order, orientation of the $k$-handles or altering the path from the base point to a $k$-handle are merely multiplying from the left by $h(E)$ where $E$ is a generalized permutation matrix, conjugates $\hat{E}_{i}$ by $h(E)$, but $\hat{F}_{i}$ is unchanged. Similarly for $(k+1)$-handles. Thus, $\theta$ is unaltered and is an invariant for our representation $\alpha$ at the beginning of this section.

The slab invariants $Z^{q}$ can be combined to form

$$
Z=\sum_{q=1}^{r} A_{q}^{-1} Z^{q}=\left[\begin{array}{c}
X \\
Y
\end{array}\right]
$$

Let us set

$$
\zeta=\operatorname{Tr} Z \in W h_{1}\left(\pi_{1} W ; \Gamma\right) /\{c+\bar{c}\} .
$$

The analysis of preceding sections implies that $\zeta$ is independent of all choices used to define $Z^{q}$. Altering order, orientation and paths to the base points of $(k+1)$-handles are multiplying from the right of $A_{q}$ by $h(E)$ where $E$ is a generalized permutation matrix. Similarly for $k$-handles. So, $\zeta$ is another invariant of our representation $\alpha$.

(J) The invariance of $\theta$ and $\zeta$. The construction of $\theta$ and $\zeta$ depends on

(1) The choice of a cobordism representation of $\alpha \in \mathscr{C}(M)$, which determines the graphic together with the singular phenomena of vertical tangent and cusps:
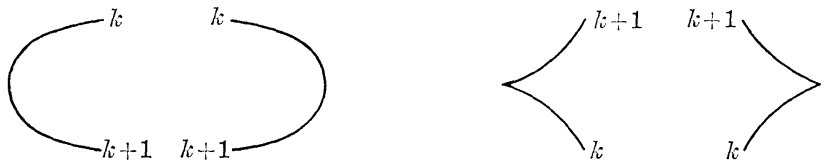

FIGURE 30 
(2) The choice of a gradient-like vector field $\xi$.

(3) The choices of $k$-, $(k+1)$-caps.

The above choices can be accomplished through a sequence of changes of the following sorts:

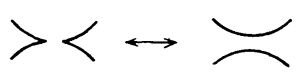

(2a) Introduction or cancellation of handle additions in a regular range.

(2b) Handle exchange.

(3a) Introduction or cancellation of regular homotopies in a regular range.

(4a) Permutations of order of any of the above phenomena.

Let us show that $\theta$ and $\zeta$ are independent of the above changes in reverse order, provided that $\operatorname{dim} \geqq 15$.

Permutation of order. Since the geometry of different cases of permutation of order is similar, let us consider only two cases. Let us first consider permutation of a regular homotopy and a $k$-handle addition. Let $E=1+E_{i j}(p)\left(p \in \pi_{1} W\right)$ be the elementary matrix corresponding to adding $p e_{i}$ to $e_{j}$. Let $[D]$ (with $D=E_{k h}(q)$, $q \in \pi_{1} W$ ) be the form of a regular homotopy between $e_{k}$ and $e_{h}$. Let us assume that the regular homotopy begins on the left. If $i, j \neq k, h$, then these phenomena are quite independent so that $E$ and $[D]$ remain unchanged by the permutation, so do $\theta$ and $\zeta$. The interesting case happens when $h=i$. Although $E$ remains unchanged by the passing, $[D]$ becomes $\left[E^{*-1} D E\right]$. This corresponds to the identity

$$
h(\widehat{E}) l([D])=l\left(\left[E^{*} D^{-1} E\right]\right) h(\widehat{E})
$$

in $\widetilde{S t U}\left(\pi_{1} W\right)$ [11] [12], and so $\theta$ is unchanged. Now consider $\zeta$. Fix all deformations at $t_{p} p \neq q+1$. Since none of the persistent intersections in $\left(t_{q}, t_{q+1}\right)$ are restricted by compatibility in the choice of deformation at $t_{q+1}$, we choose the deformation so that $\operatorname{Tr} A_{q}^{-1} X^{q}=0$. By 'translating' these choice of deformations to the situation after permutation, we have $\operatorname{Tr} A_{q}^{-1} X^{q}=0$ after permutation too, and $\zeta$ invariant. 
Permutation of a regular homotopy and a surgery. Let $(b, c ; \xi)$ be the invariants of a surgery involving $g_{1}$ and $e_{1} \cdot(\mathrm{Cf} .(\mathrm{H})$ of this section.) Let $[D]$ be the form of a regular homotopy involving $e_{k}$ and $e_{h}$ as above, which passes the surgery from right to left. Since $e_{k}$ and $e_{h}$ exist in a slice before $e_{1}$ has appeared after the permutation, $k, h>1$. We have the following picture to illustrate the situation in $\partial_{-} W$ :

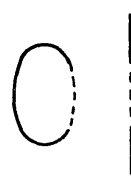

surgery

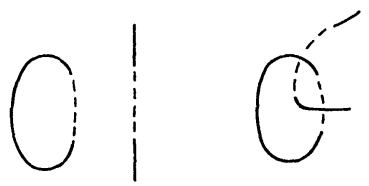

regular homotopy

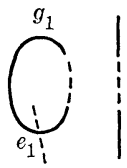

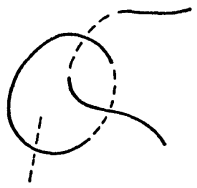

regular homotopy

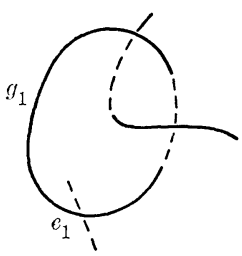

surgery

FIgURe 31

Delaying the surgery which induces $g_{1}$, until after regular homotopy, will not change $[D]$ of the regular homotopy, but will alter the data of surgery to $(b, c+P b ; \xi)$ where $P=D-(-1)^{k} D^{*}$. This corresponds to the identity

$$
l([D]) \hat{\sigma}_{11}(b, c ; \xi)=\hat{\sigma}_{11}(b, c+P b ; \xi) l([D])
$$

in $\widetilde{S t U}\left(\pi_{1} W\right)$ [11]. So, $\theta$ is invariant under this permutation. The analysis of the invariance of $\zeta$ is analogous to that of the previous case.

Introduction or cancellation of phenomena in a regular range. Consider the following list of phenomena

TABLE 1

\begin{tabular}{l|c|c}
\hline \multicolumn{1}{c|}{ Type } & Effect & Identity in $\widetilde{S t U}\left(\pi_{1} W\right)$ \\
\hline$k$-handle addition & $\begin{array}{l}p \text { times } e_{i} \text { added to } e_{j} \text { then } \\
\text { removed } \\
p \text { times } g_{j} \text { added to } g_{i} \text { then } \\
\text { removed } \\
\text { regular homotopy }\end{array} \quad \begin{array}{l}\text { regular homotopy of the } \\
\text { form }[D] \text { between } e_{i} \text { and } \\
e_{j}, \text { done and undone }\end{array}$ & $1=h\left(\hat{E}^{-1}\right) h(\hat{E})$ \\
\hline
\end{tabular}


Let us indicate the proof for the first case. Here are the pictures in $\partial_{-} W$.

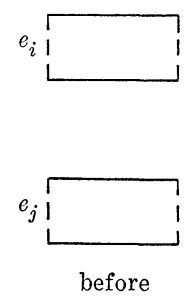

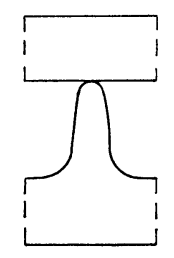

the moment of introduction

FIGURE 32

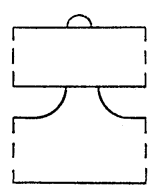

after

Geometrically, the effect is to introduce two $k$-handle additions, with the same invariant, but opposite sign, corresponding to the identity of $\widetilde{S t U}\left(\pi_{1} W\right)$ in Table 1 . As for $\zeta$, we may make the deformations symmetric so that $Z^{q}=0$. So, $\zeta$ is unchanged.

Handle exchange. Let us assume the handle exchange (cf. [3]) takes place between $e_{1}$ and $g_{1}$ with invariant $\lambda \in \pi_{1} W$. The slice invariant in $\left[t_{q}, t_{q+1}\right]$ just before the handle exchange is then of the form

$$
A_{q}=\left[\begin{array}{ll|l}
0 & a \\
b & c & * \\
\hline d & e & * \\
f & g &
\end{array}\right] .
$$

In $\partial_{-} W$ the pictures, going once around the handle exchange in a two parameter family, look like:

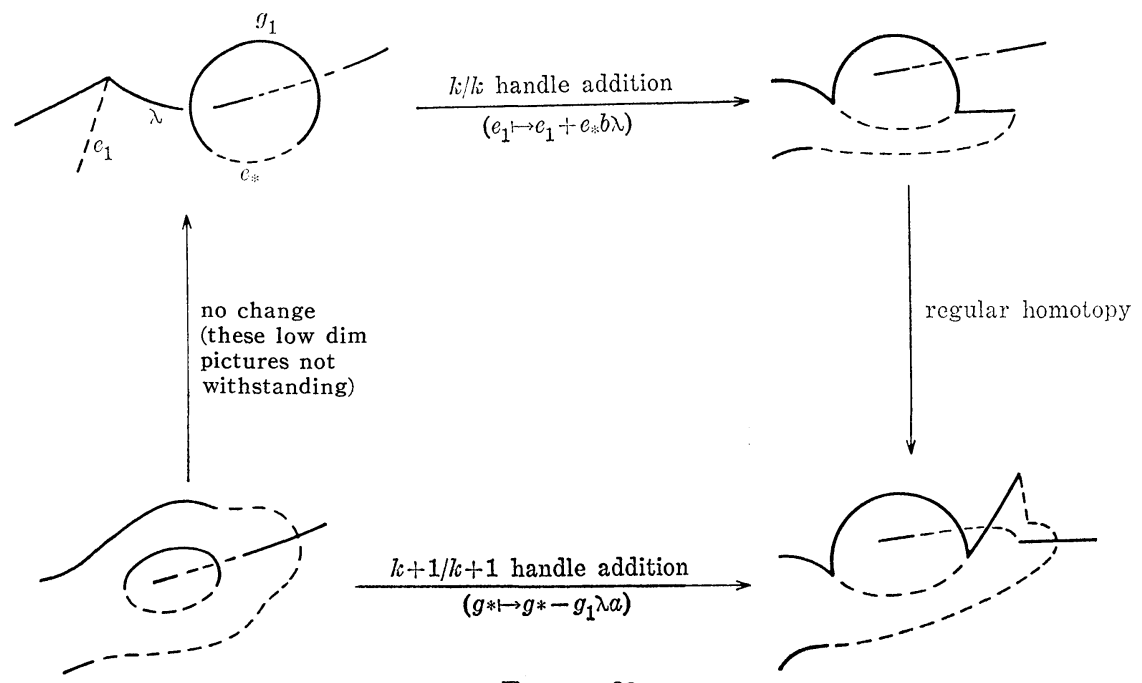

FIGURE 33 
The corresponding identity in $\widetilde{S U}\left(\pi_{1} W\right) / \widetilde{T U}\left(\pi_{1} W\right)$ is

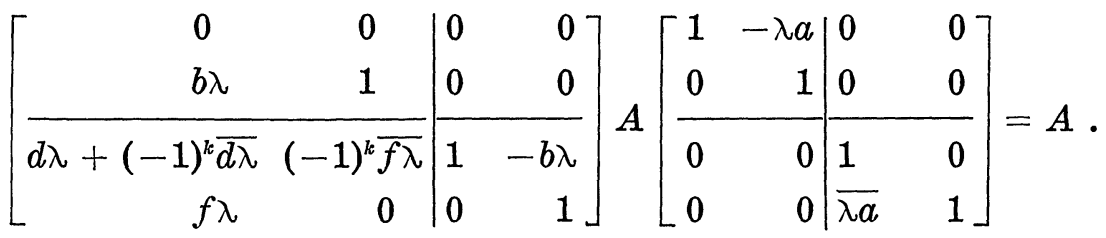

(See [11] for the definition of $\widetilde{T U}\left(\pi_{1} W\right)$ and the identity.) Since this identity lifts to $\widetilde{S t U}\left(\pi_{1} W\right)$, a handle exchange does not alter the invariant $\theta$. A similar analysis shows that $\zeta$ is not altered either.

Birth, death and surgery. Consider the following list of phenomena:

TABLE 2

Iype
$\longrightarrow$

(Note: $\tau$ is a transposition, $\sigma=\sigma(b, c ; \xi)$ Cf. (H) of this section.)

We shall only treat the second last case. (The first two cases are essentially treated in [3].)

Let us now consider the effect of
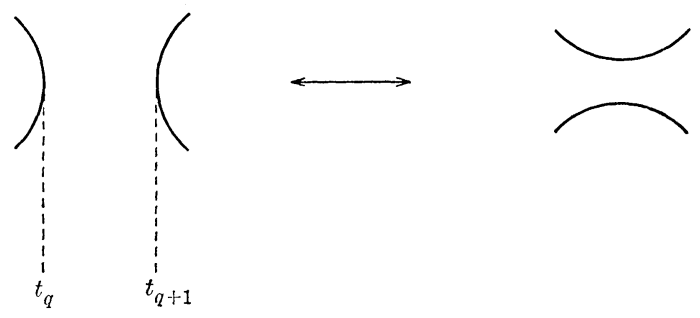

FIGURE 34

Suppose that the dying handles are first in the ordering and the rest second. Let $\tau$ be the transposition interchanging 1 and 2 , and 
let $\sigma=\sigma_{11}(b, c ; \xi)$ corresponding to the surgery death. (Cf. (H) of the present section.) Then, $\sigma^{\tau}\left(=\sigma^{H(\tau)}\right)$ corresponds to the birth, $A_{q}=\sigma^{-1} A_{q-1}, A_{q+1}=\sigma^{\tau} A_{q}$ and

$$
\theta=\hat{E} \hat{\sigma}^{\tau} \hat{\sigma}^{-1} \hat{A}_{q-1} \hat{F} \hat{A}_{s}^{-1}
$$

before the change. Note that $A_{q}$ is like the identity in the places affected by $\tau$ so that $\hat{A}_{q}^{\tau}=\hat{A}_{q}$. After the change, we have

$$
\begin{aligned}
\theta^{\prime} & =\hat{E}^{\tau} \hat{A}_{q-1} \hat{F}^{\tau} \hat{A}_{s}^{\tau-1} \\
& =\hat{E}^{\tau} \hat{\sigma} \hat{\sigma}^{-1} \hat{A}_{q-1} \hat{F}^{\tau} \hat{A}_{s}^{\tau-1} \\
& =\left(\hat{E} \hat{\sigma}^{\tau} \hat{A}_{q}^{\tau} \hat{F} \hat{A}_{s}^{-1}\right)^{\tau} \\
& =\left(\hat{E} \hat{\sigma}^{\tau} \hat{\sigma}^{-1} \hat{A}_{q-1} \hat{F} \hat{A}_{s}^{-1}\right)^{\tau} \\
& =\theta^{\tau}=\theta .
\end{aligned}
$$

Thus, $\theta$ is unchanged.

As for $\zeta$, the symmetry of this situation and $\operatorname{Tr}\left(\hat{A}_{q}^{\tau}\right)^{-1}\left(Z^{q}\right)^{\tau}=$ $\operatorname{Tr} A_{q}^{-1} Z^{q}$ make $\zeta$ unchanged.

So, we see that $\theta$ and $\zeta$ are well-defined for $\operatorname{dim} \geqq 15$.

(K) For $6 \leqq \operatorname{dim} M \leqq 14$, we prove the well-definedness as follows. Fix a simply-connected closed manifold $V^{12}$ of index and Euler characteristic 1 . If we have a cobordism representation $\alpha$

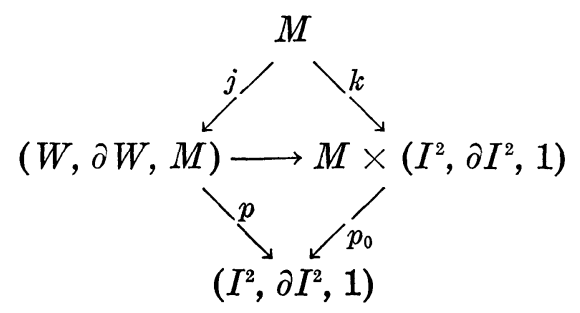

of an element $[\alpha] \in \pi_{0}(\mathscr{C}(M))$ with $M$ as the fibre, we can form the product of the cobordism with $V^{12}$ to define a representation of an element $\psi[\alpha])$ of $\pi_{0}\left(\mathscr{C}\left(M \times V^{12}\right)\right)$.

$$
\text { i: } \pi_{0}(\mathscr{C}(M)) \longrightarrow \pi_{0}\left(\mathscr{C}\left(M \times V^{12}\right)\right)
$$

is a homomorphism for $\operatorname{dim} M \geqq 6$. Let us define $\theta([\alpha])=\theta(\psi([\alpha]))$ and $\zeta([\alpha])=\zeta(\psi([\alpha])$ if $6 \leqq \operatorname{dim} M \leqq 15$. Using the product formula of [3] and [13], $\theta$ and $\zeta$ are well-defined and agree with the one parameter family definitions for $\operatorname{dim} M \geqq 6$.

LEMMA 5.1. The diagram (26) commutes for $n$ even.

Proof. This is clear except perhaps for the first square. The homomorphism $\delta: \pi_{1} \mathscr{L}_{2}(M) \rightarrow \pi_{0} \mathscr{B}(M)$ can be described as follows. 
Let $F: W^{n+3} \rightarrow M \times D^{2} \times I$ be a normal map which is a diffeomorphism over $M \times\left(D^{2} \times 0 \cup S^{1} \times I\right)$. Every class in $\pi_{1}\left(\mathscr{L}_{2}(M)\right)$ is represented by such a map, and the image under $\delta$ is represented by the restriction over $M \times D^{2} \times 1$. We shall construct a map $F$ in such a way as to realize the class of $A \in S U\left(\pi_{1} M\right)\left(\pi_{1} M=\pi_{1} W\right)$ so that the image under $\delta$ is $\delta[A] \in W h_{2}\left(\pi_{1} M\right) /\{c+\bar{c}\}$. Recall Wall's construction of [A]: Begin with $I d: M^{n} \times D^{2} \times I \rightarrow M^{n} \times D^{2} \times I$. Then add $l(k+1)$-handles $M \times D^{2} \times 1$ to trivially embedded $S^{k} \times D^{k+2}$ 's. The new end of the cobordism is an even dimensional surgery problem with a canonical subkernel. Use $A$ to get a new subkernel, and add $l$ more $(k+1)$-handlef to kill it. The resulting cobordism realizes $[A] \in L_{n+3}\left(\pi_{1} M, w\right)$.

We shall do exactly the same thing, taking care to keep track of the singularities at each stage. Each of the $l$ surgeries introduces a circle of singularities $\bigcirc_{k+1}^{k}$. If we do not put these in general position, we can see the canonical basis of the new cycles consists of: the descending manifolds of index $k+1$ critical points, and the ascending manifolds of the index $k$ critical points. However, we can place these cycles more symmetrically by the use of three swallow's tails to get:
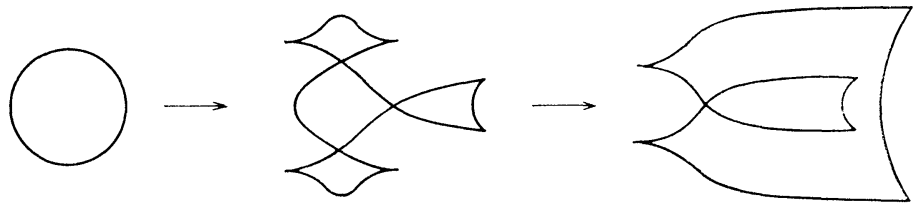

FIGURE 35

At this point, the odd dimensional invariant is

$$
h\left(\begin{array}{lr}
0 & -I \\
I & D
\end{array}\right) l\left(\begin{array}{ll}
0 & I \\
\pm I & \Sigma
\end{array}\right) h\left(\begin{array}{ll}
I & \Sigma \\
0 & I
\end{array}\right) \sigma_{22}(I, 0) h(\Sigma) l\left(\Sigma^{*}\right)
$$

where the factors are positioned as in the following picture [11] [12]:

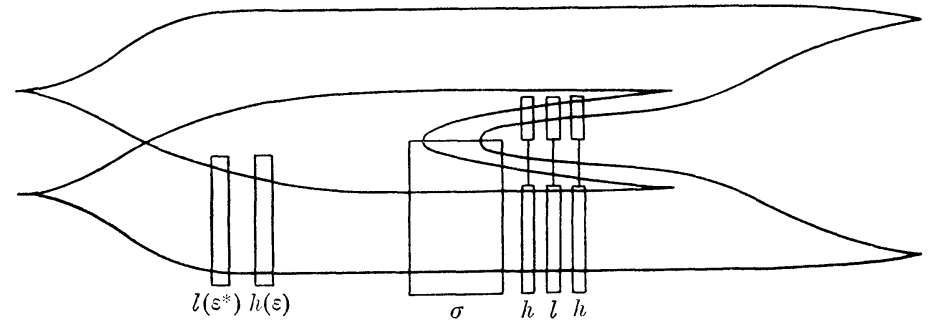

FIGURE 36

The canonical cycles now appear as descending index $(k+1)$ manifolds together with the trace of the $k$-handles over which they open 
up, as in the following picture:

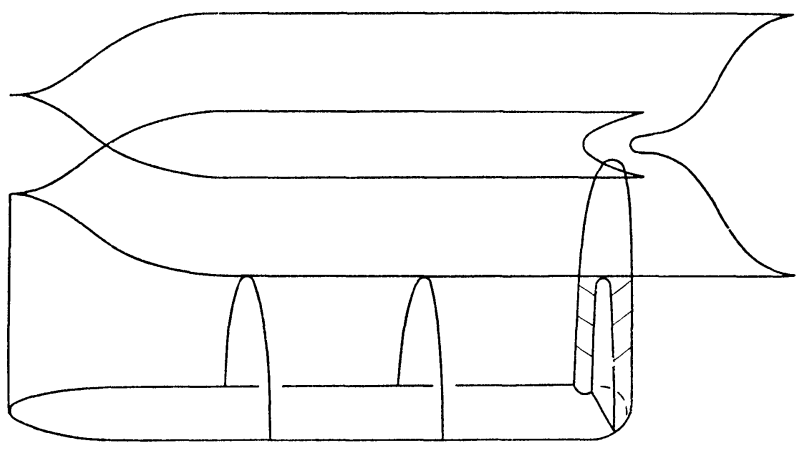

FigURE 37

Henceforth we draw the picture as

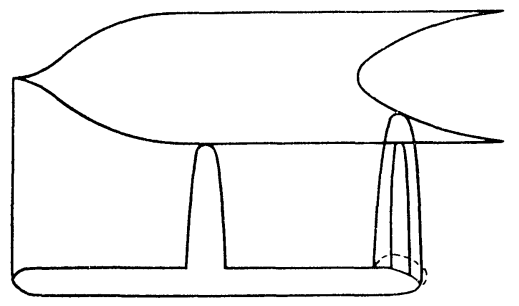

Many symmetrized circles, all superimposed on one another, and all cycles appearing symmetrically.

FigURe 38

Now, we introduce the identity

$$
\left(\begin{array}{cc}
A & \\
& A^{*-1}
\end{array}\right)\left(\begin{array}{cc}
A^{-1} & \\
& A^{*}
\end{array}\right)=I
$$

into the picture as follows

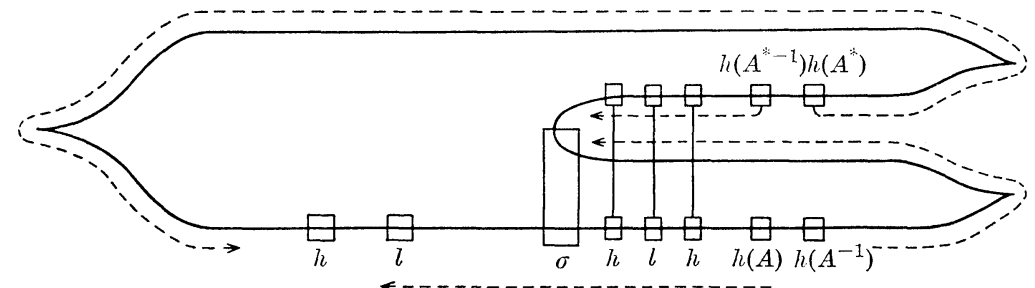

FIgURE 39

Then, the new ingredients are moved by the commutation relations [11], according to the arrows in the diagram. Because $A \in \widetilde{S U}\left(\pi_{1} M\right)$, 
and the particular form of the odd dimensional invariant, there is no change in any of the pieces of the invariant at the end of the commutation. On the left, we get

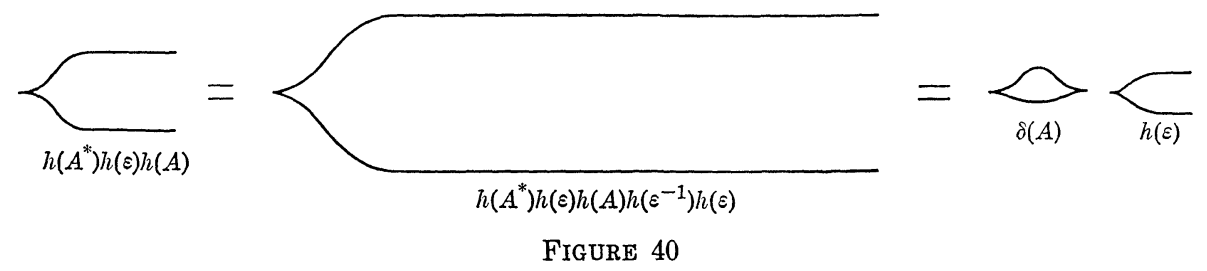

Near the surgery, we get

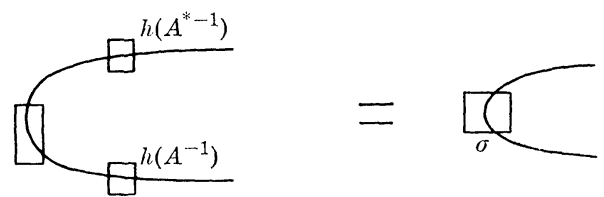

Figure 41

by repeated applications of the commutation identities. The graphic has returned to its original state, except for an addition of $\delta[A]$. Finally, we note that the effect on all of this on the canonical basis is to replace it by $A e_{1}, A e_{2} \cdots$ etc. Thus in killing the new graphic in the standard way, we realize a surgery obstruction $[A]$.

For the commutativity of (26) it remains only to observe that the $W h_{1}(\pi ; \Gamma) /\{c+\varepsilon \bar{c}\}$ invariant of elements of $\operatorname{Im}(\delta)$ is zero. This is because the invariant factors through $\pi_{0}(\mathscr{C}(M))$.

Theorem 2.1 now follows for $n$ even by the 5-lemma.

VI. Proof of Theorem 2.1 for $n$ odd. Similar to the $n$ even case, we have a cobordism representation $\alpha$ of an element $[\alpha] \in \pi_{0}(\mathscr{C}(M))$, and normal maps

$$
f_{t}: W_{t} \longrightarrow M \times\{t\} \times I
$$

if $\{t\} \times I$ contains no cusp or vertical tangent. We assume that the surgery birth and death always have the following graphics:
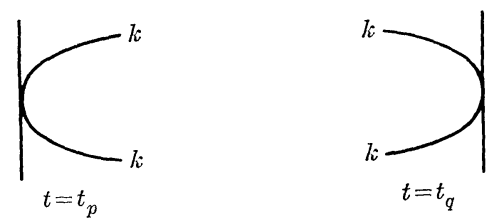

FIGURE 42 
On the other hand, the usual birth and death have the following graphics:
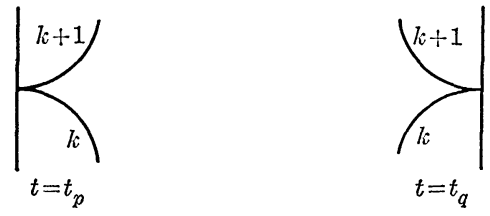

FigURE 43

At a $t$-slice slightly to the right (respectively left) of a surgery birth (respectively death), the bottom of the descending critical manifolds of two critical points of index $k$ meet $\partial W_{t}$ in two framed embedded $(k-1)$-spheres. We call them the $k$-bottoms of the surgery birth (respectively death). They bound immersed framed $k$-dises in $\partial_{-} W_{t}$ which will be called the $k$-caps of the surgery birth (respectively death). The intersections of various $k$-caps measure the linkings of $k$-bottoms, and hence the intersections of the cycles in the homology kernel of the normal map $f_{t}: W_{t} \rightarrow M \times\{t\} \times I$. In fact, we may assume that one of the pair of $k$-caps begins (respectively ends) its life as an embedded framed disc which intersects the other $k$-cap only and the intersection is an interval.

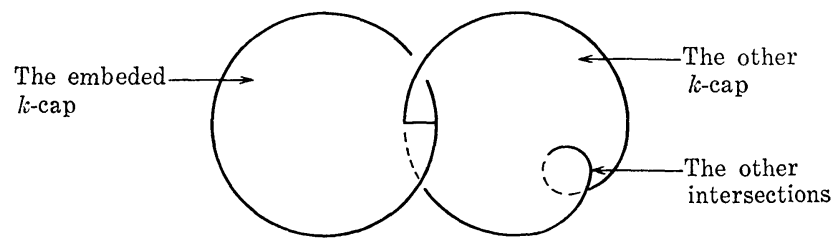

FIGURE 44

Similarly, at a $t$-slice slightly to the right (respectively left) of a usual birth (respectively death) point, the critical manifold of the critical point of index $(k+1)$ meets $\partial_{-} W_{t}$ in a $k$-disc whose boundary is the intersection of the descending manifold of index $k$ with $\partial_{-} W_{t}$, which is a framed $(k-1)$-sphere. They are $(k+1)$-bottom and $k$-bottom of the critical points respectively. Pushing the $(k+1)$ bottom off itself slightly, we can fill the $k$-bottom by an embedded framed $k$-disc as the $k$-cap of the critical point. At the beginning (respectively ending) of their lives, this cap is disjoint from the other caps. 


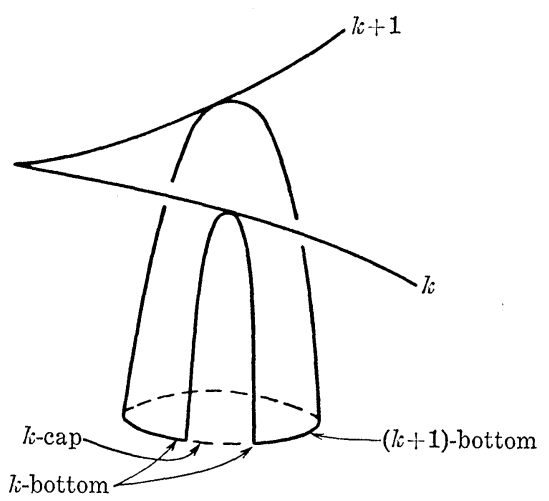

Figure 45

When $t$ moves on, we meet handle additions, surgery birth-death, the usual birth-death and regular homotopies of the caps at isolated moments. There exist $0<t_{1}<\cdots<t_{r}<1$ such that cusps, vertical tangents, handle additions and regular homotopies only occur at these times, once for each time. We have regular $t$-slices for $t \neq t_{1}, \cdots, t_{r}$. Let us now fix a base point $* \in W$ together with an orientation of $W$ at $*$, a path $\gamma(\alpha)$ from $*$ to each arc $\alpha$ of $(k+1)$ handles together with an orientation of the core disc. Insisting that the intersection number at a level surface in a slice slightly to the right of a birth point to be $+1, \gamma(a)$ induces a path to the corresponding arc of $k$-handles by going around the birth points. Order these pairs of handles at the birth point. Breaking up the arcs of $k$-handles at the surgery birth-death points, we have arcs of $k$-handles. Similarly, we join a path $\gamma(a)$ from * to each surgery birth point, together with an orientation of the core of the upper arc of $k$-handles. Insisting the linking number of the $k$-bottom of the upper handle (whose $k$-cap is an embedded framed disc) with that of the lower handle at a slice slightly to the right of the surgery point to be +1 , we have an analogous path to the corresponding lower arc of $k$-handles. Order these pairs of $k$-handles at the surgery birth points. Let $D_{1}^{k+1}, D_{1}^{k} ; \cdots ; D_{s}^{k+1}, D_{s}^{k}$ be the pairs of $(k+1)$-handles and $k$-handles created by usual birth and let $D_{s+1}^{k}, D_{s+2}^{k}, \cdots ; D_{s+2 l-1}^{k}, D_{s+2 l}^{k}$ be the pairs of $k$-handles created by surgery birth with the above ordering such that $D_{s+2 i-1}^{k}$ and $D_{s+2 i}^{k}(i=1, \cdots, l)$ are upper and lower handles respectively. Let us now divide the coordinate $t$ into subintervals $0<t_{1}<\cdots<t_{r}<1$. For $t \neq t_{i}(i=1, \cdots r), D_{j}^{k+1}$ and $D_{\imath}^{k}$ form geometric bases $g_{1}, \cdots, g_{s} ; e_{1}, \cdots, e_{s}, e_{s+1}, \cdots, e_{s+2 l}$ of the kernel chain complex of the normal map $f_{t}: W_{t} \rightarrow M \times\{t\} \times I$. We shall compute the slice invariant of the parametrized surgery problem $W_{t}$ and its change (when we pass $t=t_{i}$ ) in terms of these bases. The analysis is partly similar to that of [3] and partly similar to $\S \mathrm{V}$. We shall 
not always give so much detail as there.

(A) A regular slice. Let $W_{t}\left(t_{i}<t<t_{i}+1, i=1, \cdots, r\right)$ be a regular slice. We shall describe how the critical manifolds and caps fit together. Let $g_{j}$ be the descending manifolds of the $j$ th $(k+1)$ handle. It may meet the critical points of index $k$ several times and we have a $(k+1)$-handle $D_{j}^{k+1}$ attached along $k$-handles $e_{i},{ }_{1}, e_{i_{2}}$ etc. Note that we have no $(k+1)$-handle attached across another $k$-handle.

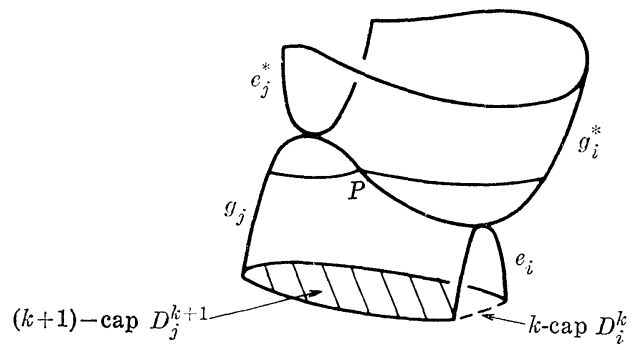

A $(k+1)$-handle is attached along a $k$-handle

FIGURE 46

$e_{j}^{*}$ is the dual handle corresponding to $g_{j}$, and $g_{i}^{*}$ is the dual handle of $e_{i}$. The critical manifolds fit together transversely along a common part $P$ which is a union of an interval, a $k$-disc and another $(k-1)$ disc. We shall mostly be concerned with this intersection at a level surface (between the two critical points involved), where $P$ is a single point intersection between a framed $k$-sphere and a framed $(k-1)$-sphere in a $(2 k-1)$-dim manifold. Next, we observe that we have intersections and self-intersections of the $k$-caps of the critical points of index $k$. They are intervals or circles (generally nontrivially covered).

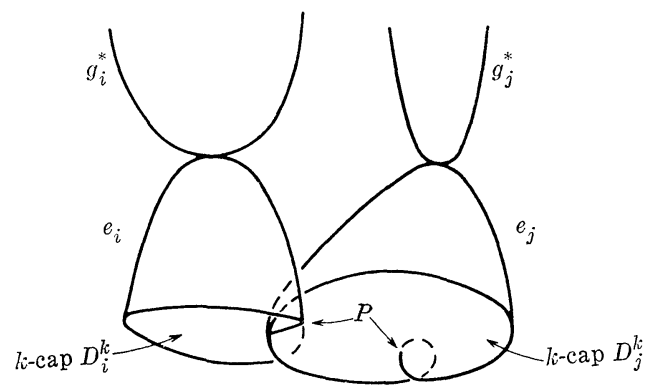

A $k$-handle is attached across another $k$-handle. $P$ denotes the intersections.

FIgURe 47

Note that $k$-handles are always attached across each other. When we follow the trajectories issueing from the $k$-caps upward and them in general position until we come to a level surface slightly above the bottom and then we follow the trajectories issueing from 
the $k$-bottoms only and go up to the critical points, we have $k$-cycles of the kernel of the normal map $f_{t}$ represented by immersed framed spheres $e_{1}, \cdots, e_{s+2 l}$. The intersections of the $k$-caps become the intersections and the self-intersections of the $e$ 's. On the other hand, the $(k+1)$-caps together with the descending manifolds of the critical points of index $(k+1)$ form punctured $(k+1)$-spheres $g_{1}, \cdots, g_{s}$. Clearly, we have $\left\{g_{1}, \cdots, g_{s}\right\}$ as a basis of the $(k+1)$-chains of the homology kernel of $f_{t}$. Now, we are ready to define the slice invariant for a regular slice.

It is a based chain complex

$$
\mathscr{C}_{i}: 0 \longrightarrow C_{k+1} \stackrel{\partial}{\longrightarrow} C_{k}
$$

with $\left\{g_{1}, \cdots, g_{s}\right\},\left\{e_{1}, \cdots, e_{s+2 l}\right\}$ as bases for $C_{k+1}$ and $C_{k}$ respectively such that

(i) $C_{k}$ has the intersection form $\lambda$ and the self-intersection form $\mu$ given by

$$
\begin{gathered}
\lambda\left(e_{i}, e_{j}\right)=\sum_{P} \varepsilon_{P} g_{P} \\
\mu\left(e_{i}\right)=\sum_{P} \varepsilon_{P} g_{P} \\
\text { (ii) } \quad \partial g_{j}=\sum_{P} \varepsilon_{P} g_{P} e_{i}
\end{gathered}
$$

summing over $P$ where $P$ are the intersections of $e_{i}$ and $e_{j}$. summing over $P$ where $P$ are self-intersections of $e_{i}$. summing over $P$ where $P$ are intersections corresponding to $g_{j}$ being attached along $e_{i}$.

(iii) $C_{k} / \partial\left(C_{k+1}\right)$ is based and the induced form $(G, \lambda, \mu)$ is a special Hermitian form representing the surgery problem

$$
f_{t}: W_{t} \longrightarrow M \times\{t\} \times I .
$$

Such a based chain complex is called a radical special Hermitian form. (Actually, $(G, \lambda, \mu)$ is visibly representing the 0 obstruction for surgery.) We take the above radical special Hermitian form as our slice invariant. For $t \in\left(t_{i}, t_{i+1}\right)$, the slice invariant $\mathscr{C}_{i}$ is independent of $t$. Altering the order, orientation and the path from the base point to the arc of critical points, the radical special Hermitian form changes like the usual special Hermitian form of [13].

For each component $C$ of $(k+1) / k$ intersection or each component of intersection of $k$-caps in $\partial_{-} W$, we have a loop $\lambda(x)$ for $x \in C$ proceding from $*$ to the $(k+1)$-handle or the first $k$-cap via the path as defined in $\S \mathrm{V}$, passing through the intersection to the $k$-handle or the second $k$-cap and then coming back to $*$ via the path as defined in $\S \mathrm{V} . \lambda(x)$ determines an element $\sigma \in \pi_{1} W$. Following [3], we define 
representatives $g(\sigma)$ for $\sigma \in \pi_{1} W$ and $\tilde{\lambda}(x)$ for $x \in C$. As we remarked before, the intersection of $k$-caps becomes points for a fixed slice, if we follow the trajectories up slightly and put them in general position. Therefore, we may view the component $C$ in $W_{t}\left(t_{i}<t<t_{i+1}\right)$ as an interval or a circle. If it is a circle, it follows from [4] that we have a map $\tilde{\lambda}(C): S^{1} \rightarrow(\Omega W)_{0}$.

Following the analysis of $\S \mathrm{V}$ and [3], we define a loop $\widetilde{\lambda}(x)$ and its contraction for $x$ being an $(k+1) /(k+1)$ intersection, $k / k$ intersection, birth-death, surgery birth-death or regular homotopy point. They occur at the singular slice for $t=t_{i}(i=1, \cdots, r)$. Using the contractions of the loops at the singular slices, we have $\tilde{\lambda}(C): S^{1} \rightarrow(\Omega W)_{0}$ for $C$ an arc of intersections. Giving the standard framing to the intersections near a singular slice, we make $\tilde{\lambda}(C)$ a map to framed cobordism. Define $A_{i}=\left(a_{h m}^{i}\right)$ with $a_{h m}^{i}=\Sigma \alpha(C) \sigma(C)$ where $C$ runs through all the components of $(k+1) / k$ intersections of the $m$ th $(k+1)$-handle with the $h$ th $k$-handle in the interval $\left(t_{i}, t_{i+1}\right)$ and $\alpha(C)$ is the element of $\Omega_{1}^{f r}\left((\Omega W)_{0}\right)$ constructed as above. $A_{i}$ is an $(s+2 l) \times s$ matrix. Next, we define $B_{i}=\left(b_{h m}^{i}\right)$ with $b_{h m}^{i}=\sum_{c} \beta(C) \sigma(C)$ where $C$ runs through all the components of intersections of the $m$ th $k$-cap with the $h$ th $k$-cap in the interval $\left(t_{i}, t_{i+1}\right)$ and $\beta(C)$ is defined as $\alpha(C) . B_{i}$ is an $(s+2 l) \times(s+2 l)$ matrix. $B_{i}$ may be viewed as the matrix of the bilinear form with coefficients in framed cobordism recording the mutual intersection data. In the next few paragraphs, we shall define elementary matrices $F_{a}, G_{a}(\alpha=1, \cdots, r-1)$ in $E(\Lambda, s)$ and $E(\Lambda, s+2 r)$ respectively. They record the change of the slice invariant as we pass the singular slices. $\left(G_{i} G_{i-1} \cdots G_{1}\right)^{-1}$ and $F_{i} F_{i-1} \cdots F_{1}$ act on $A_{i}$ from the left and the right as linear transformations. Similarly $\left(G_{i} \cdots G_{1}\right)$ acts on $B_{i}$ as a Hermitian form. We have

$$
\begin{aligned}
\alpha_{i} & =\operatorname{Tr}\left[\left(G_{i} \cdots G_{1}\right)^{-1} A_{i}\left(F_{i} \cdots F_{1}\right)\right] \\
\beta_{i} & =\sum_{j=1}^{l} b_{s+2 j-1}^{i}, s_{s+2 j}\left[\left(G_{i} \cdots G_{1}\right)^{*} B_{i}\left(G_{\imath} \cdots G_{1}\right)\right] .
\end{aligned}
$$

We define the slab invariant for $\left[t_{i}, t_{i+1}\right]$ to be the class

$$
\alpha_{i}+\beta_{i} \in W h_{1}(\pi ; \Gamma) /\{c-\bar{c}\} .
$$

(B) Geometric cancellations. Similar to $\S \mathrm{V}$, we have the following geometric cancellations: homotopy of the gradient like vector field to introduce a pair of $(k+1) / k$ intersections, regular homotopy of the $k$-caps to introduce a pair of mutual intersections or a pair of self intersections. Using the same argument of $\S \mathrm{V}$, the slice invariant and the slab invariant remain unchanged for geometric cancellations. 
(C) Handle addition I $(k / k)$.
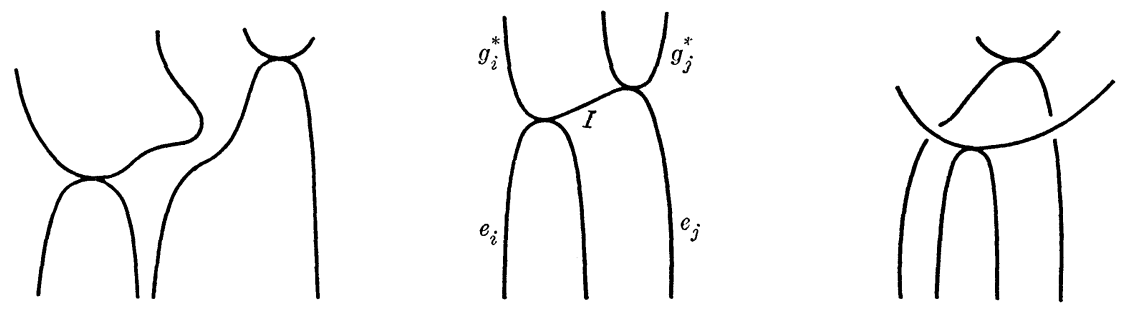

FIGURE 48

On the singular slice $t_{q}$, there is an instantaneous intersection $I$ between $g_{i}^{*}$ and $e_{j}$. If $P$ is a persistent intersection corresponding to a $(k+1)$-handle $g_{s}$ attached along $e_{j}$, or corresponding to a $k$-handle $e_{s}$ attached across $e_{i}$, then it splits into two after passing the slice, with one copy for the original intersection, and the other for the new intersection. Let $g_{I} \in \pi_{1} W$ be defined by a path from $*$ to $e_{j}$, via $I$ and $g_{i}^{*}$ and then back to *. Let $\varepsilon_{I}= \pm 1$ be defined as in $\S \mathrm{V}$ by comparing the orientation of $g_{i}^{*}$ and $g_{j}^{*}$. The effect is of multiplying the basis of $C_{k}$ from the left by the matrix.

$$
E=I+E_{i j}\left(\varepsilon_{I} g_{I}\right)
$$

with $\varepsilon_{I} g_{I}$ at the $(i, j)$ th spot, and the boundary map matrix is left multiplied by

$$
E^{-1}=I-E_{i j}\left(\varepsilon_{I} g_{I}\right) .
$$

Let $F_{q}=I, G_{q}=E^{-1}=I-E_{i j}\left(\varepsilon_{I} g_{I}\right)$ in $E(\Lambda, s+2 l)$, and let $\hat{F}_{q}, \hat{G}_{q}$ be the canonical liftings of $F_{q}$ and $G_{q}$ in $S t(\Lambda, s)$, St $(\Lambda, s+2 l)$ respectively.

The loops at the new intersections are homotopic to the product of the loop at $I$ with the loops at the old intersections, and the framings at the new intersections are gotten from the old ones. Thus, the deformations of the old intersections together with that at $I$ give us a complete set of deformations. Inductively, we have $F_{1}, \cdots, F_{q} ; G_{1}, \cdots, G_{q}$ and the slab invariant defined for $\left[t_{q}, t_{q+1}\right]$ using the above normalization of loops and the framing. The effect on $\alpha_{q-1}+\alpha_{q}$ of renormalizing at $I$ was essentially discussed in [4]. View $M_{q-1}=G_{q-1} \cdots G_{1}$ and $M_{q}=G_{q} M_{q-1}$ as elements of $G L(\Lambda, s+2 l)$ where $G_{q}=I+E_{i j}\left(\varepsilon_{I} g_{I}\right)$. Changing by the amount $\alpha \in \Gamma$ leads to a discrepancy of $\alpha_{q-1}+\alpha_{q}$ equal to

$$
\operatorname{Tr}\left(M_{q-1}^{-1} E_{i j}\left(\alpha \varepsilon_{I} g_{I}\right) M_{q-1}\left(\frac{I}{0}\right)\right)=\operatorname{Tr}\left(((I) 0) M_{q-1}^{-1} E_{i j}\left(\alpha \varepsilon_{I} g_{I}\right) M_{q-1}\right)
$$

where $I$ is the $(s \times s)$-matrix with 1 along the diagonal. Let us now consider the effect on $\beta_{q-1}+\beta_{q}$ of the above renormalization. Write 


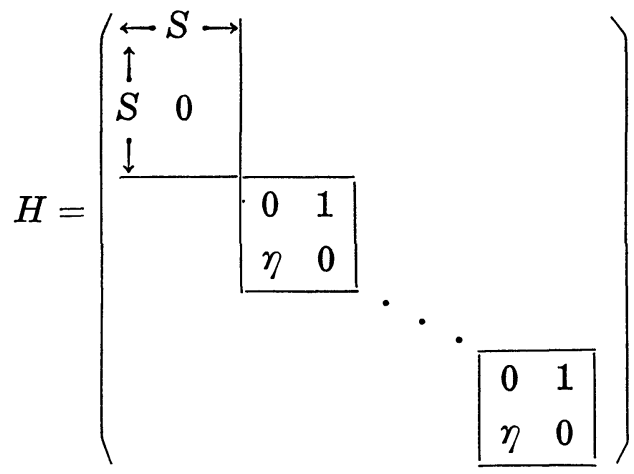

with $s$ zeros on the diagonal and $l$ blocks of $\left(\begin{array}{ll}0 & 1 \\ \eta & 0\end{array}\right)\left(\eta=(-1)^{k}\right)$. It represents the standard radical special Hermitian form. $B_{q-1}+B_{q}$ is changed by

$$
H M_{q-1}^{-1} E_{i j}\left(\alpha \varepsilon_{I} g_{I}\right) M_{q-1}+\eta\left(H M_{q-1}^{-1} E_{i j}\left(\alpha \varepsilon_{I} g_{I}\right) M_{q-1}\right)^{*} .
$$

So, $\beta_{q-1}+\beta_{q}$ is changed by an amount

$$
\sum_{j=1}^{l} b_{s+2 j-1}, s+2 j\left[H M_{q-1}^{-1} E_{i j}\left(\alpha \varepsilon_{I} g_{I}\right) M_{q-1}+\eta\left(H M_{q-1}^{-1} E_{i j}\left(\alpha \varepsilon_{I} g_{I}\right) M_{q-1}\right)^{*}\right]
$$

The total change of $\alpha_{q-1}+\alpha_{q}+\beta_{q-1}+\beta_{q}$ in $W h_{1}\left(\pi_{1} ; \Gamma\right) /\{c-\bar{c}\}$ is equal to

$$
\operatorname{Tr}\left(M_{q-1}^{-1} E_{i j}\left(\alpha \varepsilon_{I} g_{I}\right) M_{q-1}\right) \quad \bmod \{c-\bar{c}\}
$$

which is zero. So, the slab invariant is unchanged under a renormalization at $I$.

(D) Handle addition II $((k+1) /(k+1))$. This is essentially discussed in [3]. Similar to (C), we have an instantaneous intersection $I$ at the singular slice for $t=t_{q}$ and $g_{I} \in \pi_{1} W, \varepsilon_{I}= \pm 1$. The effect is multiplying the basis of $C_{k+1}$ from the left by the inverse of the elementary matrix

$$
E=I-E_{i j}\left(\varepsilon_{I} g_{I}\right)
$$

with $\varepsilon_{I} g_{I}$ at the $(i, j)$ th spot. This right multiplies the boundary matrix by $E^{-1}$. We put $F_{q}=E$ and $G_{q}=I$. Let $\hat{F}_{q}, \hat{G}_{q}$ be the canonical liftings of $F_{q}, G_{q}$ in $\operatorname{St}(\Lambda, s), \operatorname{St}(\Lambda, s+2 l)$ respectively.

The effect on the slab invariant is as follows. The changes of $A_{q-1}, A_{q}$ are given in [4]. So, $\alpha_{i}(i \neq q-1, q), \alpha_{q-1}+\alpha_{q}$ are unchanged under renormalization. $\beta_{i}(i=1, \cdots, q)$ are unchanged under a renormalization at the $(k+1) /(k+1)$ intersection.

(E) Regular homotopy. These introduce new intersection points 
in pairs among the $k$-caps. The algebraic sum of these intersection is zero, so there is no effect on the radical special Hermitian form and we set $\hat{F}_{q}=I, \widehat{G}_{q}=I$. Also, changing normalizations has no effect on $\alpha, \beta$.

(F) Birth-death. This is treated in [3]. Let us consider the birth case only. At the singular slice $t=t_{q}$, we add a pair of basis elements, one for $C_{k+1}$ and another for $C_{k}$ with $\lambda, \mu$ stablized in the obvious way. So, it is a stablization for the radical special Hermitian form. We again set $\hat{F}_{q}=I, \widehat{G}_{q}=I$. It follows from [4] that the slab invariant is unchanged under renormalization.

(G) Surgery birth-death. This is actually similar to the usual birth-death. At the surgery birth slice $t=t_{q}$, we create a pair of basis elements for $C_{k}$ but $C_{k+1}$ is unchanged. The first basis element intersects the second one geometrically exactly once but has no other intersections. However, the second one may have other mutual intersections and self-intersections. The effect is first stablizing $C_{k}$ by adjoining a dual pair of basis element and then replacing $e_{s+2(l+1)}$ by its sum with a linear combination of $e_{i}(i \leqq s+2 l)$. This has the effect of multiplying the stabilized basis on the left by an elementary matrix $G_{q}$, which has a canonical lifting $\hat{G}_{q} \in \operatorname{St}(\Lambda, s+2(l+1))$.

By a similar argument of [4], the slab invariant is unchanged when we renormalize a surgery birth-death slice.

(H) The invariants $\theta$ and $\zeta$. In the preceding paragraphs, we have defined the slice invariant of a representative $\alpha$ of an element $[\alpha] \in \pi_{0}(\mathscr{C}(M))$ as the radical special Hermitian form $\mathscr{C}_{q-1}$ in the $\left(t_{q-1}, t_{q}\right)$. It changes to $\mathscr{C}_{q}$ by the formula

$$
\mathscr{C}_{q}=G_{q} \mathscr{C}_{q-1} F_{q}^{-1}
$$

where the action of $G_{q}$ and $F_{q}^{-1}$ are interpreted as changes of basis in dimensions $k$ and $k+1$ as we pass through the singular slice $t=t_{q}$. Furthermore, $F_{q}, G_{q}$ are elementary matrices with specific liftings $\hat{F}_{q}, \hat{G}_{q}$ in $\operatorname{St}(\pi)$. Let us examine the image $A$ of the product

$$
\hat{G}_{r} \cdots \hat{G}_{1} \hat{F}_{1}^{-1} \cdots \hat{F}_{2}^{-1}
$$

in $E(\pi)$. Recall that $\mathscr{C}_{0}$ is identified with the standard radical special Hermitian form

$$
0 \longrightarrow C_{k+1} \stackrel{\partial}{\longrightarrow} C_{k} \longrightarrow 0
$$

with $\left\{g_{1}, \cdots, g_{s}\right\},\left\{e_{1}, \cdots, e_{s}, e_{s+1}, \cdots, e_{s+2 l-1}, e_{s+2 l}\right\}$ as the bases for $C_{k+1}$ and $C_{k}$ respectively such that $\partial g_{i}=e_{i}(i=1, \cdots, s)$, and $\left\{e_{s+1}, \cdots, e_{s+2 l}\right\}$ form the standard basis for the special hyperbolic form (kernel [13]) 
of $C_{k} / \partial C_{k+1}$. There exists a generalized permutation matrix $W$ such that $B=W A$ is of the form

$$
\left(\begin{array}{cc|c}
\uparrow & \\
s & I & * \\
\downarrow & * \\
\hline 0 & U
\end{array}\right)
$$

such that $U \in \widetilde{S U}\left(\pi_{1} M\right)$. Thus $\hat{W} \hat{G}_{r} \cdots \hat{G}_{1} \hat{F}_{1}^{-1} \cdots \hat{F}_{r}^{-1} \in \mathscr{P}$ and so determines a class $\theta \in L_{2 k+1}^{s t}(\pi, w)$ of the element $[\alpha] \in \pi_{0}(\mathscr{C}(M))$. Next we define the invariant $\zeta$ of $[\alpha] \in \pi_{0}(\mathscr{C}(M))$ to be

$$
\sum_{i=1}^{r-1}\left(\alpha_{i}+\beta_{i}\right) \in W h_{1}(\pi ; \Gamma) /\{c-\bar{c}\}
$$

where $\alpha_{i}, \beta_{i}$ are the slab invariants defined for $\left[t_{i}, t_{i+1}\right](i=1, \cdots, r-1)$.

We claim that $\theta$ and $\zeta$ are well-defined. First, we observe that it follows from [3] that changing of the vector field, the regular homotopies of $k$-caps, and permutations of basis elements, etc. essentially change the element in $\mathscr{P}$ by a specific lifting of an element of $\widetilde{E U}(\pi) \rightarrow E(\pi)$. These changes do not affect the graphic and the invariants $\theta$ and $\zeta$.

We also have to check the changes listed in $\S \mathrm{V}(\mathrm{J})$. Following the arguments of [3] and $\S V$, they do not affect $\theta$ and $\zeta$. Let us only remark about the change of the graphic
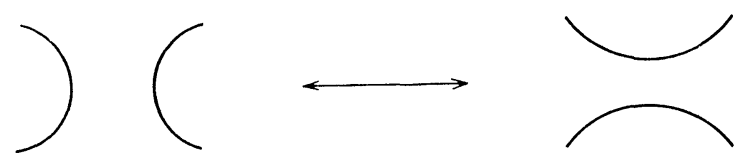

Figure 49

as a sample. In this case, the argument is similar to the change of the graphic
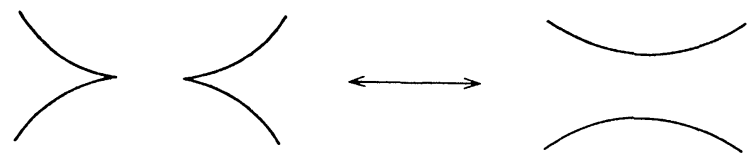

FIGURE 50

as given in [3]. Let us consider the change 


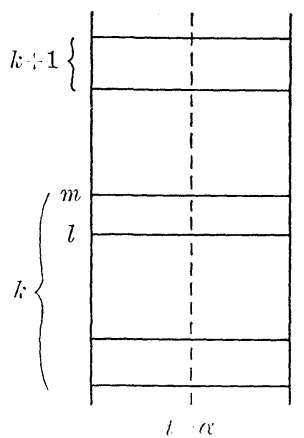

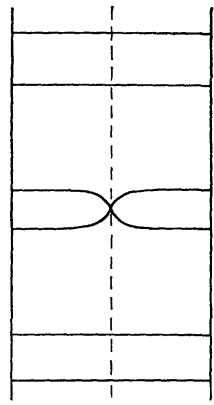

$t=-\alpha$

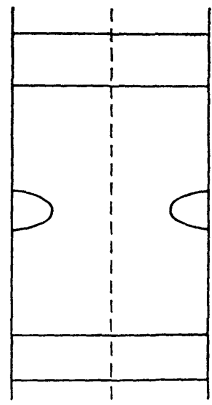

$t \cdots \kappa$

Figure 51

Let $\hat{F}_{1}, \cdots, \hat{F}_{q-1}, \hat{G}_{1}, \cdots, \widehat{G}_{q-1}$ be the elements in the Steinberg group constructed from the left most graphic up to $t=\alpha$. Let

$$
\mathscr{C}_{q-1}: 0 \longrightarrow C_{k+1}^{(q-1)} \stackrel{\partial}{\longrightarrow} C_{k}^{(q-1)} \longrightarrow 0
$$

be the radical special Hermitian form for $t=\alpha-\delta(\delta$ small $)$. We may assume that they are the same both the left most and the right most graphics. We see that $\lambda\left(e_{m}, e_{l}\right)=\varepsilon g\left(g \in \pi_{1}(W), \varepsilon= \pm 1\right)$, $\mu\left(e_{m}\right)=0$ and $e_{m}$ has no intersections with other $e$ 's. After a suitable permutation of the basis elements and left multiplying by

$$
\left(\begin{array}{cc|c}
\stackrel{\uparrow}{\uparrow} & \\
s & I & 0 \\
\downarrow & \\
\hline 0 & A
\end{array}\right)
$$

where $A \in \widetilde{T U}(\Lambda, l)$ (cf. [11], [12]), we may assume that $\mu\left(e_{l}\right)=0$ and $e_{l}$ has no intersections with other $e$ 's. Therefore, we may view the changes of the radical special Hermitian forms as follows. When it passes $t=\alpha-(1 / 2) \delta, \mathscr{C}_{q-1}$ is changed to $\mathscr{C}_{q}$ by left multiplying a generalized permutation matrix together with an element of the form (110). Denote its lifting in $S t(\pi, s+2 l)$ by $\hat{G}_{q}$ and set $\hat{F}_{q}=I$. Then, we move across $t=\alpha+(1 / 2) \delta$ and $\mathscr{C}_{q}$ is changed to $\mathscr{C}_{q+1}$ with $\hat{G}_{q+1}=\hat{G}_{q}^{-1}$ and $\hat{F}_{q+1}=I$.

Let us now describe the radical special Hermitian forms for the right most graphic. We may set $\mathscr{C}_{i}(i \leqq q)$ equal to those for the left most graphic. We can get $\mathscr{C}_{q+1}$ as follows. (a) Relabel $e_{m}$ as $e_{s+u}(u$ odd) where $s+u, s+u+1$ have not occurred as indices yet. Relabel $e_{l}$ as $e_{s+u+1}$. (b) Let the base path for $e_{s+u}$ be the old base path for $e_{m}$. It induces a new base path for $e_{s+u+1}$. Note that the difference between the old base path for " $e_{l}$ and the new 
one for $e_{s+u+1}$ is $g^{-1}$. (c) Orient $e_{s+u}$ and $e_{s+u+1}$ properly such that $\lambda\left(e_{s+u}, e_{s+u+1}\right)=+1$. These normalizations define $\hat{F}_{q+1}^{\prime}, \widehat{G}_{q+1}^{\prime}$ for the right most graphic. Set $\hat{F}_{i}^{\prime}=\hat{F}_{i}, \hat{G}_{i}^{\prime}=\widehat{G}_{i}$ for $i \leqq q$. So, we have $\hat{F}_{1}^{\prime}, \cdots, \hat{F}_{q+1}^{\prime}, \hat{G}_{i}^{\prime}, \cdots, \hat{G}_{q+1}^{\prime}$ and we can follow a similar argument as the birth-death relation of [3] to show that $\theta$ is unchanged under this change of the graphic. It is clear that $\zeta$ is also unchanged.

So, we have a homomorphism

$$
\phi=\theta \oplus \zeta: \pi_{0}(\mathscr{C}(M)) \longrightarrow L_{2 k+1}^{s t}(\pi, w) \oplus W h_{1}(\pi ; \Gamma) /\{c-\bar{c}\} .
$$

We remark that all of the changes we have considered explicitly have no effect on the classes $\alpha, \beta$ in $W h_{1}\left(\pi_{1} ; \mathbf{Z}_{2} \oplus \pi_{2}\right) /\{c-\bar{c}\}$. We wish to indicate here how a 'bad eye' can be converted to a circle, by the following sequence of changes:

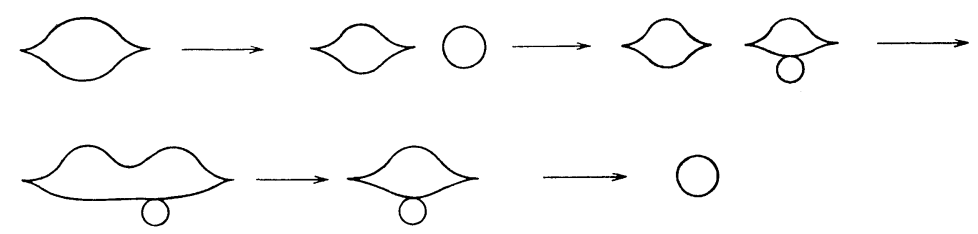

Figure 52

(I) The homomorphism $\delta: \pi_{1}\left(\mathscr{L}_{2}(M)\right) \rightarrow \pi_{0}(\mathscr{C}(M))(n+1=2 k+2)$. Let us consider an element $[\alpha] \in \pi_{1}\left(\mathscr{L}_{2}(M)\right)$ represented by a normal map $F: W^{n+3} \rightarrow M \times D^{2} \times I$ which is a diffeomorphism over $M \times\left(D^{2} \times 0 \cup S^{1} \times I\right)$ and a simple homotopy equivalence on the boundary. We shall construct the image of $[\alpha]$ under $\delta$ by the restriction over $M \times D^{2} \times 1$. Recall the construction of a representative $\alpha$ of $[\alpha] \in \pi_{1}\left(\mathscr{L}_{2}(M)\right)=L_{2 k+4}(\pi, w)$ [13]. Begin with Id: $M \times D^{2} \times I \rightarrow M \times D^{2} \times I$. Suppose that $\alpha$ is represented by a special Hermitian form with $\left\{e_{1}, \cdots, e_{l}\right\}$ as the basis. Choose $l$ disjoint discs $D_{i}^{2 k+3} \subset \operatorname{Int}\left(M \times D^{2} \times 1\right)$ and let $f^{0}: S^{k+1} \times D^{k+2} \subset D^{2 k+3}$ be the standard embedding, so by composition we have $l$ disjoint embeddings $f_{i}^{0}: S^{k+1} \times D^{k+2} \subset \operatorname{Int}\left(M \times D^{2} \times 1\right)$. We now deform the $f_{i}^{0}$ by regular homotopies $\eta_{i}$ to new disjoint embeddings $f_{i}^{\prime}$ such that the intersections of the traces of the regular homotopies realizing the given form. Attaching $l(k+2)$-handles on $f_{\imath}^{\prime}$, we have the realization of the given element of $L_{2 k+4}(\pi, w)$. Using the results of $\S \mathrm{V},[11]$ and [12], we can lift $\alpha$ to $\overparen{S t U}(\pi)$ representing an element of $L_{n+1}^{s t}(\pi, w)$ such that it projects back to $[\alpha] \in L_{n+3}(\pi, w)$. The geometric meaning is as follows. We can choose a product structure $D^{2}=I \times I$, and a cobordism representation 


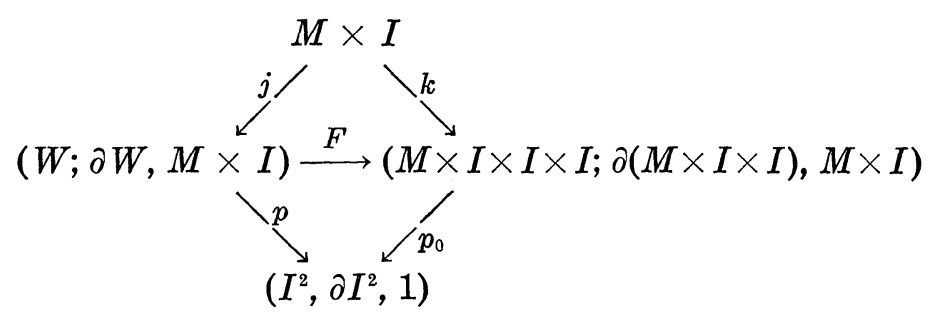

of $\pi_{0}(\mathscr{C}(M \times I$ rel $M \times \partial I))$ where $p_{0}$ is the projection of the last two factors of $I$. The projection $p$ is factored through

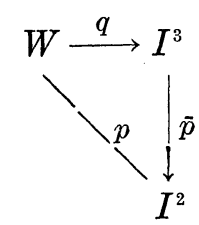

where $I^{3}$ is the original $D^{2} \times I$ and $\bar{p}$ is the standard projection. We may identify $p^{-1}(I \times 0), p^{-1}(0 \times I)$ as $M \times I \times I \times 0, M \times I \times 0 \times I$ and $p$ as the standard projections on them. Since $p$ has no critical points over $\partial I^{2}$, we may assume that $q$ has no critical points over $I \times I \times 0 \cup I \times 0 \times I \cup I \times I \times 1$ and its graphic has no vertical tangent over $I \times 1 \times I$.

Let us now consider a regular slice over $I \times t \subset I^{2}$. We have a commutative diagram

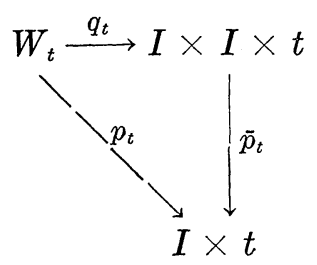

Following $\S \mathrm{V}$, we have an odd dimensional surgery problem

$$
W_{t} \stackrel{F_{t}}{\longrightarrow} M \times I \times I \times t
$$

represented by an element $A_{t} \in \widetilde{S U}(\pi)$. Actually, it follows from [13] and $\S \mathrm{V}$ that $A_{t}$ tells how to attach handles on $M \times I \times 0 \times t=$ $p_{t}^{-1}(0 \times t)$ to get $W_{t}$. Following the construction, we have an $s$-cobordism $W_{1 t}=p_{t}^{-1}(1 \times t)$ over $M \times 0 \times 1 \times t$. It is practically visible that $W_{1 t}$ has only $k-,(k+1)$-handles and $(k+2)$-handles on $M \times 0 \times 1 \times t$ and the $s$-cobordism is represented by the same matrix $A_{t} \in E(\pi)$ when we use these handles as basis. As we pass a singular slice over $I \times t_{i}, A_{t} \in \widetilde{S U}(\pi)$ is changed by an element in $\widetilde{S t U}(\pi)$ and the $s$-cobordism is changed by its image in $S t(\pi)$. This 
change can be achieved by handle additions on the $s$-cobordism. Now, we may make $q$ generic and $\bar{p} \cdot q$ representing the same $\widetilde{S t U}(\pi)$ data. Moreover, we may require that the graphic of

$$
W_{1} \stackrel{q_{1}}{\longrightarrow} I \times 1 \times I
$$

has no vertical tangent and represents a pseudo-isotopy with its data given by the image of $A_{t}$ in $S t(\pi)$. This pseudo-isotopy representation clearly represents $\delta([\alpha]) \in \pi_{0}(\mathscr{B}(M))$. It is not difficult to see that $\delta([\alpha])$ is well-defined and its image is always in $W h_{2}\left(\pi_{1}(M)\right) /$ $\{c-\bar{c}\}$. It follows from $\S I$ and the above construction that the geometric and the algebraic definition of $\delta$ coincide. Since we have shown that the diagram (26) is commutative, by the 5-lemma $\phi_{2}$ is an isomorphism and (26) is an isomorphism of exact sequences. This completes the proof of Theorem 2.1 for the case $n$ odd.

\section{REFERENCES}

1. W. Browder, Diffeomorphisms of 1-connected manifolds, Trans. Amer. Math. Soc., 128 (1967), 155-163.

2. J. Cerf, La stratification naturelle des espaces de fonctions differentiables rèelles et le théorème de la pseudo-isotopie, Publ. Math. I.H.E.S., 39 (1970).

3. A. Hatcher and J. Wagoner, Pseudo-isotopies of compact manifolds, Part I, Astérisque 6, Soc. Math. de France.

4. A. Hatcher, Pseudo-isotopies of compact manifolds, Part II, Astérisque 6, Soc. Math. de France.

5. C. Giffen, Hasse-Witt for $(\alpha, u)$-reflexive Forms and Automorphisms I, (To appear).

6. W. C. Hsiang, On the Codim 1 Isotopy Problem, Proc. of International Conference on Manifolds and Related Topics in Topology, Manifolds-Tokyo, (1973), 117-122.

7. W. C. Hsiang, J. L. Shaneson and Fake Tori, Topology of Manifolds, Proc. of the Univ. of Georgia Topology of Manifold Institute, pp. 18-51, Markham Publishing Co. 1969. 8. J. W. Milnor, Introduction to Algebraic K-Theory, Annals of Math. Studies 72, Princeton University Press.

9. S. P. Novikov, Algebraic constructions and properties of hermitian analogues of K-theory over rings with involution, Izvestija, 4 (1970), No. 2.

10. F. Quinn, A Geometric Formulation of Surgery, Princeton University Thesis, 1969.

11. R. W. Sharpe, On structure of the unitary Steinberg group, Ann. of Math., 96 (1972), 444-479.

12. - Surgery on compact manifolds: The bounded even dimensional case, Ann. of Math., 97 (1973), 187-209.

13. C. T. C. Wall, Surgery on Compact Manifolds, Academic Press, 1970.

14. H. Whitney, Singularities of mappings of Euclidean spaces, Sym. Inter. de Top. Alg. Mexico City (1958), 285-301.

Received October 31, 1975. The first author is partially supported by NSF Grant GP-34324X1, and a Guggenheim Fellowship. The second author is partially supported by NSF Grant GP-33019X.

Princeton University

Columbia UnIVERSity

AND

UNIVERSITY OF TORONTO 



\section{PACIFIC JOURNAL OF MATHEMATICS}

\section{EDITORS}

RICHARD ARENS (Managing Editor)

University of California

Los Angeles, California 90024

\section{J. DugunduI}

Department of Mathematics University of Southern California Los Angeles, California 90007

D. Gilbarg and J. Milgram

Stanford University

Stanford, California 94305

\author{
R. A. Beaumont \\ University of Washington \\ Seattle, Washington 98105
}

\section{ASSOCIATE EDITORS}
B. H. NeUmanN
F. WOLF
K. YosHIDA

\section{SUPPORTING INSTITUTIONS}

\author{
UNIVERSITY OF BRITISH COLUMBIA \\ CALIFORNIA INSTITUTE OF TECHNOLOGY \\ UNIVERSITY OF CALIFORNIA \\ MONTANA STATE UNIVERSITY \\ UNIVERSITY OF NEVADA \\ NEW MEXICO STATE UNIVERSITY \\ OREGON STATE UNIVERSITY \\ UNIVERSITY OF OREGON \\ OSAKA UNIVERSITY
}

\author{
UNIVERSITY OF SOUTHERN CALIFORNIA \\ STANFORD UNIVERSITY \\ UNIVERSITY OF HAWAII \\ UNIVERSITY OF TOKYO \\ UNIVERSITY OF UTAH \\ WASHINGTON STATE UNIVERSITY \\ UNIVERSITY OF WASHINGTON \\ AMERICAN MATHEMATICAL SOCIETY
}

The Supporting Institutions listed above contribute to the cost of publication of this Journal, but they are not owners or publishers and have no responsibility for its content or policies.

Mathematical papers intended for publication in the Pacific Journal of Mathematics should be in typed form or offset-reproduced, (not dittoed), double spaced with large margins. Please do not use built up fractions in the text of your manuscript. You may however, use them in the displayed equations. Underline Greek letters in red, German in green, and script in blue. The first paragraph or two must be capable of being used separately as a synopsis of the entire paper. Items of the bibliography should not be cited there unless absolutely necessary, in which case they must be identified by author and Journal, rather than by item number. Manuscripts, in triplicate, may be sent to any one of the editors. Please classify according to the scheme of Math. Reviews, Index to Vol. 39. All other communications should be addressed to the managing editor, or Elaine Barth, University of California, Los Angeles, California, 90024.

The Pacific Journal of Mathematics expects the author's institution to pay page charges, and reserves the right to delay publication for nonpayment of charges in case of financial emergency.

100 reprints are provided free for each article, only if page charges have been substantially paid. Additional copies may be obtained at cost in multiples of 50 .

The Pacific Journal of Mathematics is issued monthly as of January 1966. Regular subscription rate: $\$ 72.00$ a year (6 Vols., 12 issues). Special rate: $\$ 36.00$ a year to individual members of supporting institutions.

Subscriptions, orders for back numbers, and changes of address should be sent to Pacific Journal of Mathematics, 103 Highland Boulevard, Berkeley, California, 94708.

PUBLISHED BY PACIFIC JOURNAL OF MATHEMATICS, A NON-PROFIT CORPORATION

Printed at Kokusai Bunken Insatsusha (International Academic Printing Co., Ltd.), 8-8, 3-chome, Takadanobaba, Shinjuku-ku, Tokyo 160, Japan. 


\section{Pacific Journal of Mathematics}

Patricia Andresen and Marvin David Marcus, Weyl's inequality and

quadratic forms on the Grassmannian .......................

George Bachman and Alan Sultan, Regular lattice measures: mappings and

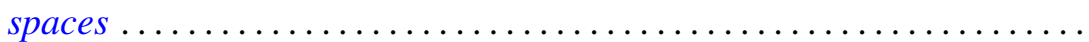

David Geoffrey Cantor, On certain algebraic integers and approximation by rational functions with integral coefficients ...................

James Richard Choike, On the value distribution of functions meromorphic in the unit disk with a spiral asymptotic value ..................

David Earl Dobbs, Divided rings and going-down................ 353

Mark Finkelstein and Robert James Whitley, Integrals of continuous

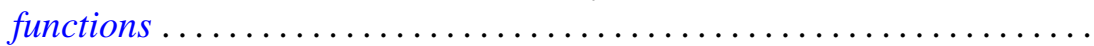

Ronald Owen Fulp and Joe Alton Marlin, Integrals of foliations on manifolds with a generalized symplectic structure ...............

Cheong Seng Hoo, Principal and induced fibrations .................

Wu-Chung Hsiang and Richard W. Sharpe, Parametrized surgery and

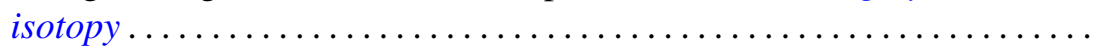

Surender Kumar Jain, Surjeet Singh and Robin Gregory Symonds, Rings whose proper cyclic modules are quasi-injective .................

Pushpa Juneja, On extreme points of the joint numerical range of commuting normal operators...

Athanassios G. Kartsatos, Nth order oscillations with middle terms of order $N-2$.

John Keith Luedeman, The generalized translational hull of a

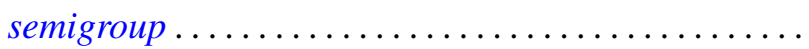

Louis Jackson Ratliff, Jr., The altitude formula and DVR's ...

Ralph Gordon Stanton, C. Sudler and Hugh C. Williams, An upper bound for the period of the simple continued fraction for $\sqrt{D}$...

David Westreich, Global analysis and periodic solutions of second order systems of nonlinear differential equations...

David Lee Armacost, Correction to: "Compactly cogenerated LCA

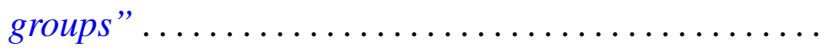

Jerry Malzan, Corrections to: "On groups with a single involution" .

David Westreich, Correction to: "Bifurcation of operator equations with unbounded linearized part" ...................... 